\title{
Semaphorin 3A induces cytoskeletal paralysis in tumor- specific CD8+ T cells
}

\author{
Mike B Barnkob ${ }^{1}$ Yale S Michaels², Violaine André ${ }^{1}$, Philip S Macklin, Uzi Gileadi ${ }^{1}$, \\ Salvatore Valvo ${ }^{4}$, Margarida Rei ${ }^{1}$, Corinna Kulicke ${ }^{1}$, Ji-Li Chen1, Vitul Jain ${ }^{5}$, Victoria \\ Woodcock ${ }^{1}$, Huw Colin-York ${ }^{1}$, Andreas V Hadjinicolaou ${ }^{1}$, Youxin Kong ${ }^{5}$, Viveka Mayya ${ }^{4}$, \\ Joshua A Bull ${ }^{6}$, Pramila Rijal ${ }^{1}$, Christopher W Pugh ${ }^{3}$, Alain R Townsend ${ }^{1}$, Lars R Olsen ${ }^{7}$, \\ Marco Fritzsche ${ }^{1,4}$, Tudor A Fulga ${ }^{2}$, Michael L Dustin ${ }^{4}$, E Yvonne Jones ${ }^{5}$, Vincenzo \\ Cerundolo'.
}

${ }^{1}$ MRC Human Immunology Unit, MRC Weatherall Institute of Molecular Medicine, University of Oxford, Headley Way, Oxford OX3 9DS, UK.

2 MRC Weatherall Institute of Molecular Medicine, University of Oxford, Headley Way, Oxford OX3 9DS, UK.

3 Nuffield Department of Medicine, University of Oxford, Nuffield Department of Medicine Research Building, Roosevelt Drive, Oxford OX3 7FZ, UK.

${ }^{4}$ Kennedy Institute of Rheumatology, University of Oxford, Roosevelt Dr, Oxford OX3 7FY, UK.

${ }^{5}$ Division of Structural Biology, Wellcome Centre for Human Genetics, University of Oxford, Roosevelt Drive, Oxford, OX3 7BN, UK.

6 Wolfson Centre for Mathematical Biology, Mathematical Institute, University of Oxford, Radcliffe Observatory Quarter, Woodstock Road, Oxford, OX2 6GG, UK.

7 Department of Health Technology, Technical University of Denmark, Ørsteds Plads, Building 345C, 2800 Kgs. Lyngby, Denmark.

Corresponding author: Vincenzo Cerundolo (V.C.), vincenzo.cerundolo@imm.ox.ac.uk.

Short title: Sema3A induces paralysis in CD8+ T cells. 
30 Keywords: cancer, immune system, T cells, semaphorin, neuropilin-1, immunotherapy, 31 cytoskeleton.

32

33 Word and character counts: Title (75 characters). Short title (40 characters). Abstract (115

34 words). One sentence summary (120 characters). Main text (3810 words). Discussion (1079 35 words). 


\section{ABSTRACT}

37 Semaphorin-3A (Sema3A) regulates tumor angiogenesis, but its role in modulating antitumor immunity is unclear. We demonstrate that Sema3A secreted within the tumor

39 microenvironment (TME) suppresses tumor-specific CD8+ T cell function via Neuropilin-1 40 (NRP1), a receptor that is upregulated upon activation with T cells' cognate antigen.

41 Sema3A inhibits T cell migration, assembly of the immunological synapse, and tumor killing.

42 It achieves these functional effects through hyper-activating the acto-myosin system in $\mathrm{T}$ 43 cells leading to cellular paralysis. Finally, using a clear cell renal cell carcinoma patient 44 cohort, we demonstrate that human tumor-specific CD8+ T cells express NRP1 and are 45 trapped in Sema3A rich regions of tumors. Our study establishes Sema3A as a potent 46 inhibitor of anti-tumor immunity. 


\section{INTRODUCTION}

Cytotoxic CD8+ T cells are often restricted to certain areas within tumors or completely excluded from the tumor microenvironment (TME) (1). We hypothesized that cell guidance cues involved in developmental processes may also play a role in $\mathrm{T}$ cell restriction in the tumor microenvironment. The secreted protein Sema3A is known to guide both endothelial cells and neurons during embryogenesis through the cell-surface receptor family Plexin-A $(2,3)$. Sema3A binding to Plexin-A requires the co-receptor NRP1 $(4,5)$. In axonal growth cones, Sema3A signaling leads to profound changes in filamentous actin (F-actin) cytoskeletal organization (6), an effect that is thought to be dependent on myosin-IIA activity (7). Sema3A can also be produced by cancer cells (8) and recent evidence indicates that NRP1, like PD-1, is upregulated on dysfunctional tumor-specific CD8+ T cells and can modulate their anti-tumor response $(9-11)$. However, there is no consensus on whether the Sema3A-NRP1 axis is immunosuppressive $(8,12)$ or supportive of CD8+ T cells' response to tumors (13). Furthermore, due to Sema3A's anti-angiogenic effects (14), several groups have proposed utilizing Sema3A to inhibit tumor growth $(13,15)$. It is therefore critical to examine the role of Sema3A in anti-tumor immunity more closely.

\section{RESULTS}

\section{Tumor-specific CD8+ T cells upregulate NRP1 and Plexin-A1}

To establish whether Sema3A can affect CD8+ T cells, we first examined NRP1 expression of its cognate receptor, NRP1 on naive and stimulated T cells. NRP1 was upregulated on human NY-ESO-1-specific HLA-A2 restricted CD8+ T cells, as well as on murine OT-I CD8+ T cells (OT-I T cells), upon stimulation with their cognate peptides, NY-ESO-1 157-165 and Ovalbumin 257-264 (Ova), respectively (Figure 1A-B). Analysis of transcriptional data from the Immunological Genome Project Consortium (16) of naive and effector CD8+ T cells corroborated these findings (Supplementary Figure 1A). We examined whole OT-I T-cell protein lysate and found that two NRP1 isoforms exist in murine T cells, with the larger NRP1 protein being the dominant form following T cell activation (Supplementary Figure 1B). To examine NRP1 regulation in CD8+ T cells, we utilized antigenic Ova peptides with varying affinities for the OT-I TCR (17), namely SIINFEKL (N4), SIIQFEKL (Q4) and SIITFEKL (T4) 
and found that NRP1 expression was correlated with both peptide concentration and affinity of TCR engagement (Figure 1C).

NRP1 is a co-receptor for a number of cell-surface receptors, including TGF $\beta$ receptors 1 and 2 (TGFßR1-2) (18), VEGF receptor 2 (VEGFR2) (19) and Plexin-A1, -A2, -A3 and -A4 receptors (20), and its function is highly dependent on the availability of these receptors for downstream signaling. We therefore screened OT-I T cells for expression of NRP1 partner receptors. Stimulated, but not naive, OT-I T cells expressed Plexin-A1 but little to no PlexinA2, TGF $\beta R 1$, TGF $\beta R 2$ or VEGFR2 (Supplementary Figure 1C-E). Plexin-A4 was expressed at low levels on both unstimulated and stimulated cells. Analysis of Plexin-A3 expression was not included because antibodies specific to Plexin-A3 could not be found. Having identified NRP1 and Plexin-A1 receptors on stimulated, but not naive T cells, we expected Sema3A ligation to the former (5). Indeed, flow cytometric analysis confirmed that only stimulated OT-I T cells could bind recombinant murine Sema3As-P (Figure 1D). Confocal imaging further indicated that Sema3A was internalized upon binding to $T$ cells (Figure 1E).

We next explored whether NRP1 and Plexin-A1 expression would be retained by CD8+ T cells during infiltration in the TME. We adoptively transferred congenically marked and activated OT-I T cells into syngeneic C57BL/6 mice bearing either B16.F10 or OVA expressing B16.F10 cells (B16.F10.Ova) in opposing flanks. While few NRP1 expressing OT-I T cells infiltrating B16.F10 control tumors were NRP1 positive, the majority of OT-I T cells residing within B16.F10.Ova tumors expressed NRP1 (Figure 1F) and Plexin-A1 (Figure 1G, right) up to eleven days after adoptive transfer. Of note, endogenous CD4+CD25+FoxP3+ T cells found within the tumor expressed both NRP1 and Plexin-A1 as well as TGFßR1-2 (Supplementary Figure 1E), indicating that this subset of $\mathrm{T}$ cells might be modulated differently from CD8+ T cells. Collectively, these data show that NRP1 and Plexin-A1 receptors are upregulated on CD8+ T cells in a TCR-dependent manner, that they are expressed on tumor-specific OT-I T cells, and that recombinant Sema3A can bind directly to activated CD8+ T cells. 
A

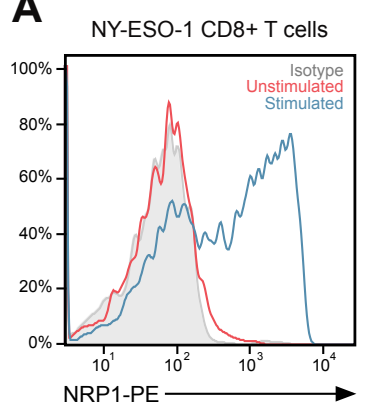

E

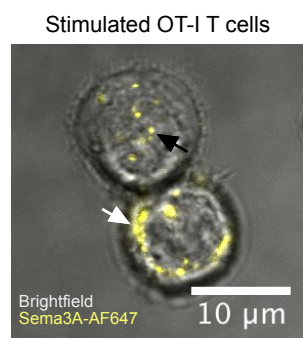

B

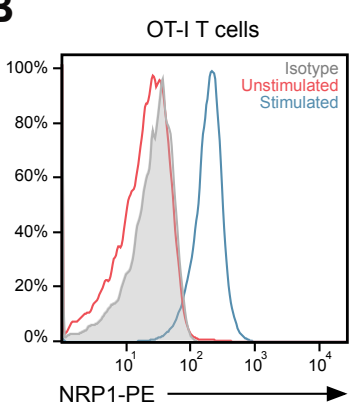

$\mathbf{F}$

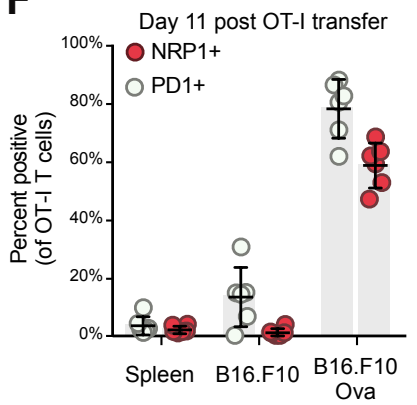

C

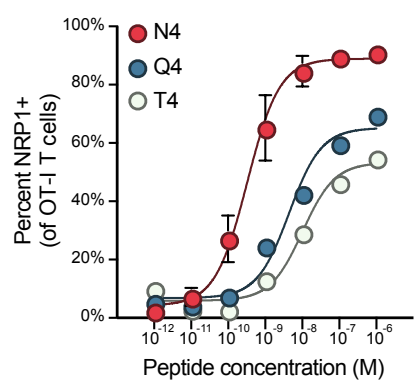

G NRP1 interaction partners

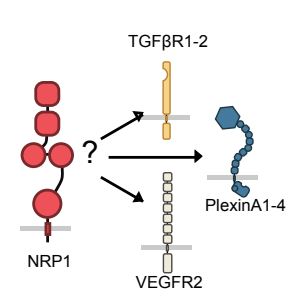

D

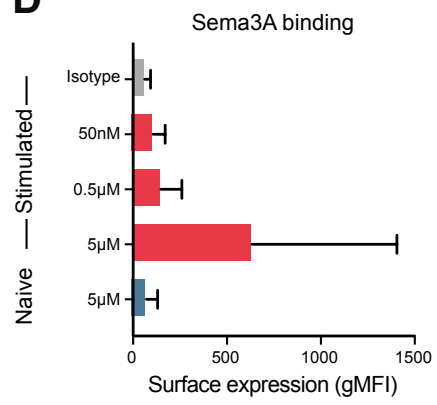

Day 11 post OT-I transfer

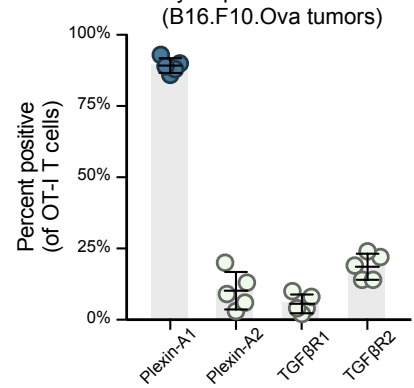

Figure 1. Tumor-specific CD8+ T cells up-regulate NRP1 and Plexin-A1 allowing for Sema3A binding.

A-B. Representative histogram of flow cytometric analysis of surface NRP1 expression on human NY-ESO-1-specific HLA-A2 restricted CD8+ T cells and murine OT-I CD8+ T cells following 48 hours stimulation with cognate peptides. Cells are gated on CD45, CD8 and TCR 3 . Experiment repeated three times. C. Analysis of NRP1 up-regulation using peptides with varying TCR affinities. Cells are gated on CD45.1, CD8 and TCR 3 . Cells from 3 mice per group, experiment was performed once. Data indicate mean \pm SD. D. Quantification of surface binding of Sema3AS-P on naïve and 48 hour stimulated OT-I T cells. Cells are gated on CD8 and CD3. Experiment was repeated three times. Data indicate mean \pm SD of representative experiment. E. Confocal imaging of 48 hour stimulated OT-I T cells stained with AF647-labelled Sema3AS-P shows that the protein can bind to the cell membrane (white arrow) and within the cell (black arrow). F. Flow cytometric analysis of PD-1 and NRP1 expression on OT-I T cells 11 days after adoptive transfer in spleen, non-antigen expressing tumor (B16.F10) and antigen-expressing tumor (B16.F10.Ova) ( $n=6)$. Data representative of two independent experiments and indicate mean \pm SD of six mice per group. G. Schematic of NRP1 interactions partners (left). Flow cytometric analysis of expression of selected NRP1 interactions partners on OT-I T cells 11 days after adoptive transfer ( $\mathrm{n}=5$ ) (right). Experiment was performed once. Data indicate mean \pm SD. Abbreviations: gMFI, geometric mean fluorescence intensity. N4, SIINFEKL. Q4, SIIQFEKL. T4, SIITFEKL.

Sema3A negatively regulates CD8+ T cell adhesion, motility and migration through

NRP1

Sema3A is known to restrict neuronal migration (4), but can have opposing effects on immune cell motility. While both thymocyte (21) and macrophage (22) migration can be inhibited, Sema3A has also been shown to increase dendritic cell (DC) migration (23). We therefore undertook a number of in vitro experiments designed to dissect the effect of Sema3A on CD8+ $T$ cell adhesion and motility. We first utilized interference reflection microscopy (IRM) to assess T cell contact and adhesion (24). This was done on plates coated with ICAM-1 and the chemokine ligand C-X-C motif chemokine ligand 12 (CXCL12, SDF-1a) in order to emulate the environment found on endothelial cells and extracellular matrix within the TME (25). When Sema3As-p was coated on plates, T cell adhesion was significantly weakened (Figure 2A), an effect that was present from initial attachment until at least 10 minutes later (Figure 2B). In addition $T$ cells displayed a reduced polarized 
122 morphology (Figure 2C, Supplementary Figure 2A). T cell motility was also affected, as 123 both distance and velocity were reduced when Sema3As-P was present, an effect that could 124 be reverted by pre-treating $T$ cells with a blocking anti-NRP1-antibody (Figure 2D-E). 125 Extravasation into tumors requires $\mathrm{T}$ cells to first adhere to endothelial cells and then 126 transmigrate into the underlying parenchyma. To model this, we performed two experiments. 127 First, we perfused T cells across surfaces with ICAM-1 and CXCL12 with or without 128 Sema3As-p, and found that under a range of external flow rates, Sema3A decreased the 129 number of cells able to display rolling or tight adhesion (Figure 2F-G). At flow rates of 80 $130 \mu \mathrm{m} / \mathrm{sec}$, many $\mathrm{T}$ cells had a migration path similar to laminar flow indicating little ability to 131 adhere (Figure 2F, lower right figure). Secondly, using a transwell assay, we found that 132 Sema3A strongly inhibited transmigration (Figure $\mathbf{2 H}$ ). We wondered if these effects were 133 mediated through changed expression levels of integrins or selectins involved in adhesion 134 and extravasation. However, flow cytometric analysis did not reveal any down-regulation of 135 CD11a (part of LFA-1), CD49d or CD162 (Supplementary Figure 2B), suggesting that 136 Sema3A signaling does not affect expression of these archetypal adhesion receptors on 137 CD8+ T cells. These data illustrate that Sema3A strongly inhibits activated CD8+ $T$ cell 138 adhesion and motility, an effect that can be modulated using anti-NRP1-blocking antibodies. 


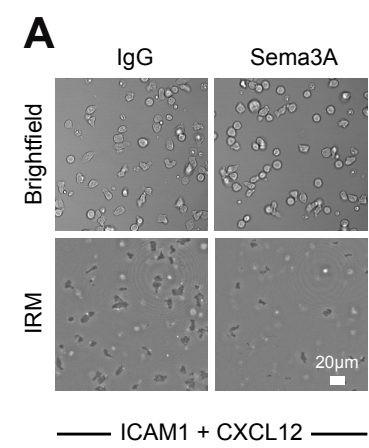

D
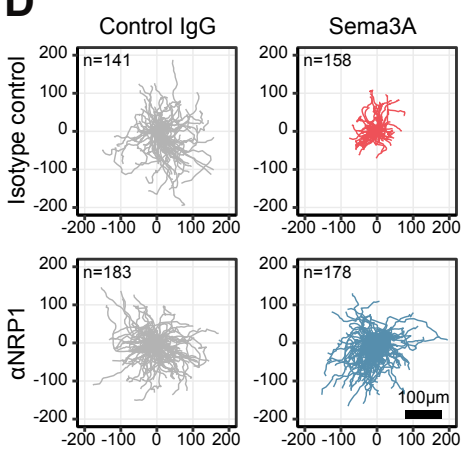

ICAM1 + CXCL12

\section{$\mathbf{F}$}
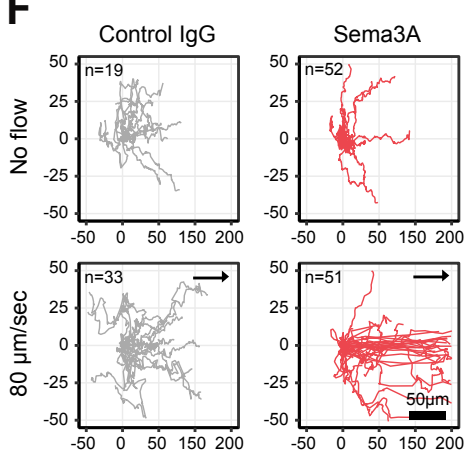
cell-cell contact
B
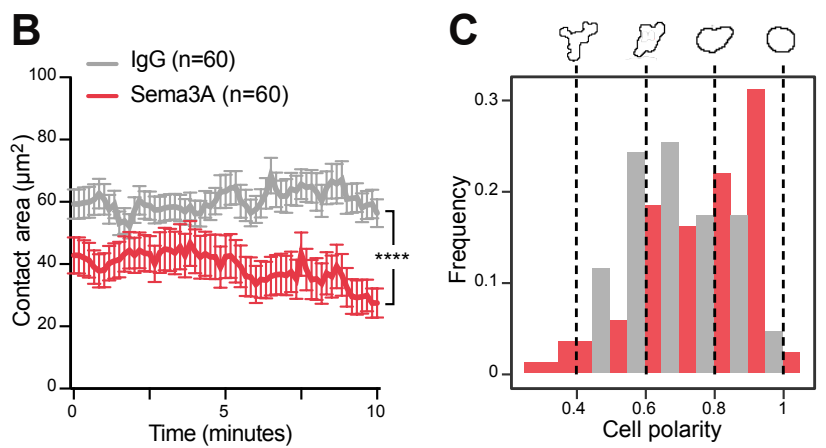

E

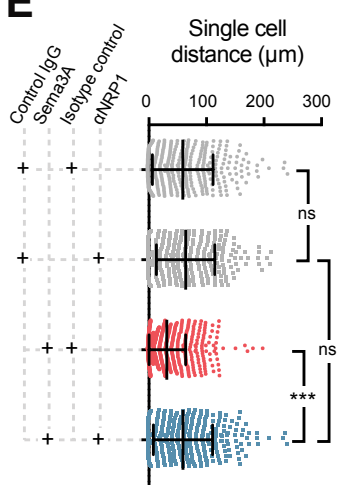

G

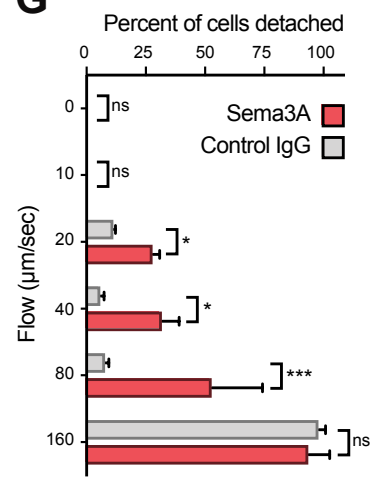

Single cell velocity $(\mu \mathrm{m} / \mathrm{min})$

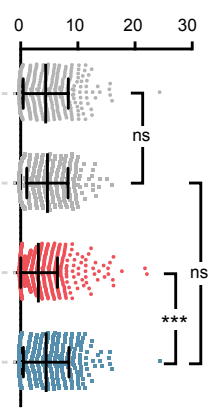

H

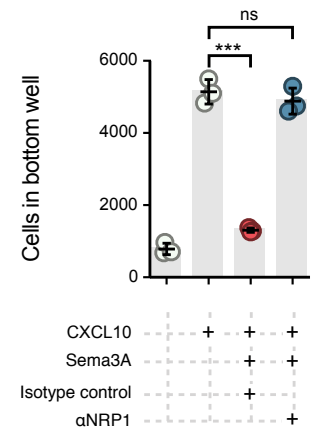

Figure 2. Sema3A negatively regulates CD8+ $T$ cell adhesion, motility and migration through NRP1.

A. Representative brightfield and IRM images of 48 hour stimulated OT-I T cells adhering to ICAM-1 and CXCL12 coated plates with either Sema3AS-P or IgG immobilized. B. Quantification of contact area per single cell using live-cell microscopy for 10 minutes after OT-I T cells were added to plate ( $n=60$ cells). Data representative of three independent experiments and indicate mean \pm SEM. ${ }^{* * *}$

$=\mathrm{P}<0.0001$ by Student's t-test. $\mathbf{C}$ Relative frequency of cell polarity from brightfield images. A polarity of 1 indicates a shape of a perfect circle, 0 a rectangular shape. Representative images of OT-I T cells illustrated above graph. D Representative spider plots showing the migration paths of individual $T$ cells pretreated with either a NRP1 blocking antibody or isotype control antibody on similar plates as in (A). E. Graph of single cell distance (left) and single cell velocity (right) in same experiment as (D). ( $n=314-744$ cells per group). Data combined from five independent experiments indicate mean $\pm \mathrm{SD}$. ${ }^{* * *}=\mathrm{P}<$ $0.001, \mathrm{~ns}=$ not significant by Kruskal-Wallis test. F. Representative spider plots showing the migration path of individual OT-I T cells on similar plates as in $(A)$, with flow rates at 0 or $80 \mu \mathrm{m} / \mathrm{sec}$. Arrows indicate flow direction. G. Quantification of percent of OT-I cells that detach in same experiment as ( $F)$ ( $n=20-73$ cells per condition). Data representative of two independent experiments and indicate mean \pm SEM. * $\mathrm{P}<0.05,{ }^{* * *}=\mathrm{P}<0.001, \mathrm{~ns}=$ not significant by two-way ANOVA. H. Representative graph of number of stimulated OT-I T cells able to transmigrate through $3 \mu \mathrm{m}$ Boyden chamber with CXCL12 in bottom chamber, with or without Sema3AS-P in top-chamber. OT-I T cells were pre-treated with either a blocking NRP1 antibody or isotype control antibody. Data representative of two independent experiments and indicate mean $\pm \mathrm{SD}$. ${ }^{* * *}=$ $\mathrm{P}<0.001$, ns $=$ not significant, by two-way ANOVA. Abbreviations: IRM, interference reflection microscopy. Sec, second.

\section{Sema3A negatively regulates CD8+ T cells' immunological synapse formation and}

Given the strong effects of Sema3A on CD8+ $T$ cell adhesion and motility, we investigated whether Sema3A also affects the formation of the immunological synapse (IS). We first tested the ability of CD8+ $T$ cells to form close contacts with an activating surface displaying immobilized ICAM-1 and anti-CD3 antibodies. To mimic an environment in which Sema3A had been secreted, $T$ cells were added and allowed to settle in medium containing either Sema3As-p or control IgG, while the size and spreading speed of contact areas was measured using time-lapse IRM. T cells added to Sema3A-rich medium formed fewer and 
smaller contact zones (Figure 3A, left, Movie S1-2). We noticed that cells in Sema3A-rich medium did not spread as much and were slower to adhere (Figure 3A, right). Indeed, when analyzing contact zones over time, many cells in Sema3A-rich medium could not form large contact areas (Figure 3B, top) and spread at a reduced velocity (Figure 3B, bottom).

These results were reminiscent of the effects seen when $T$ cells were added to plates coated with ICAM-1, CXCL12 and Sema3As-P (Figure 2A) and indicated that T cells' ability to form IS could be compromised as well.

To more closely examine the effects of Sema3A on IS formation, we utilized supported lipid bilayers containing ICAM-1, CD80 and H-2 Kb-Ova pMHC monomers. Stimulated OT-I T cells were pretreated with fluorescently-labelled Sema3As-P-I, washed to ensure that residual protein did not interfere with the bilayer, and IS formation visualized using time-lapse total internal reflection fluorescence (TIRF) microscopy. T cells with none to little Sema3A-binding were seen to form classical IS containing a CD80-clustered central supramolecular activation cluster (CSMAC) and an outer ICAM-1-rich peripheral supramolecular activation cluster (pSMAC), while T cells that had strongly bound Sema3As-P-ı were unable to spread and appeared incapable of engaging with CD80 and ICAM-1 on the bilayer (Figure 3C, Movie S3). To quantify the extent of this defect, we turned to a recently developed highthroughput method to quantify relevant IS parameters (26), where T cells are first fixed on the bilayer, then washed to remove non-adherent cells. Nearly two-thirds of stimulated T cells were either washed away, could not cluster CD80, or form pSMACs when pre-treated with Sema3As-P-ı compared to untreated T cells (Figure 3D-E). Diminished IS formation in the presence of Sema3A mirrored a scenario where OT-I T cells were presented to an irrelevant pMHC-ligand, $\mathrm{H}-2 \mathrm{~K}^{\mathrm{d}}$-gp33, on the bilayer (Supplementary Figure 3A). Among the Sema3A-treated $\mathrm{T}$ cells that formed IS, there was a discernible reduction in CD80 accumulation and in the radial symmetry of the synapse (Supplementary Figure 3B-D), indicating that CD8+ $T$ cells can be rendered non-responsive to their cognate antigen through Sema3A signaling. We confirmed these findings by examining $T$ cell binding to live cancer cells. Stimulated OT-I T cells and B16.F10.Ova cells were co-incubated in the presence of control IgG, Sema3As-p or a mutated Sema3A protein, in which the NRP1 interaction site on Sema3A has been mutated to substantially reduce the binding affinity (5), 
182 followed by enumeration of OT-I T cell:B16.F10.Ova cell-cell conjugates. We noticed a 50\% 183 reduction in number of OT-I cells capable of binding to antigen-expressing cancer cells in 184 the presence of Sema3A, but not with the control or mutant Sema3A (Supplementary 185 Figure 3E). These results thus demonstrate that Sema3A signaling leads to profound effects on a majority of CD8+ T cells' abilities to adhere to target cells and form an IS.

A

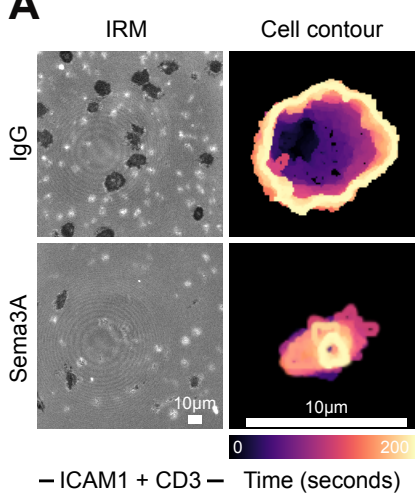

C

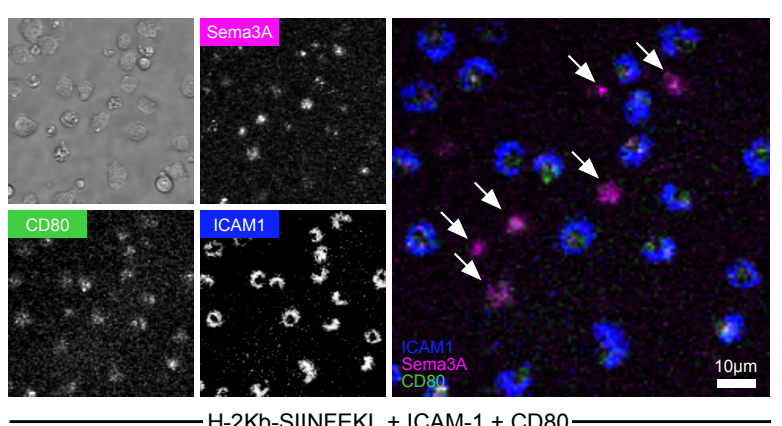

D
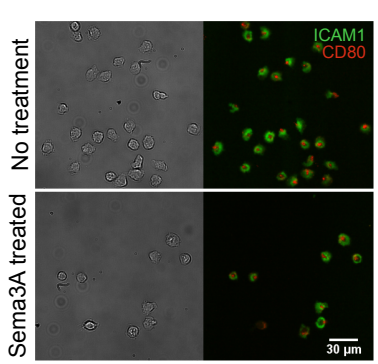

$\mathrm{H}-2 \mathrm{~Kb}-\mathrm{SIINFEKL}+$ ICAM-1 + CD80
B

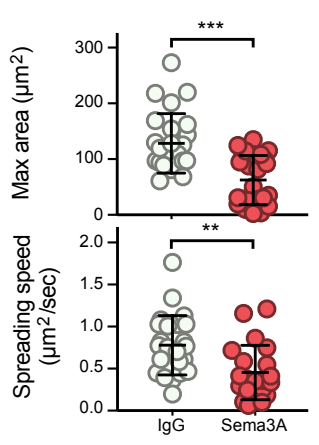

Condition
E

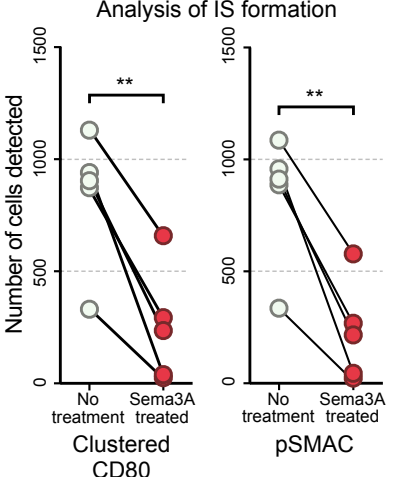

Figure 3. Sema3A negatively regulates CD8+ T cells' immunological synapse formation.

A. Live-cell imaging visualizing surface interface using IRM of stimulated CD8+ T cells dropped on an activating surface with immobilized ICAM-1 and CD3 and Sema3AS-P or lgG present in medium (left). Cell contour of representative cells from either condition (right). Color of contour indicates time from 0 to $200 \mathrm{sec}$ as denoted on scalebar. B. Quantification of maximum size of cell contact area (top) and spreading speed from initial contact to maximum contact area (bottom) $(n=25$ cells per group) in same experiment as (A). Data combined from three independent experiments and indicate mean $\pm \mathrm{SD}$. ${ }^{* *}=\mathrm{P}<0.01,{ }^{* \star *}=\mathrm{P}<0.001$ by Mann-Whitney test. C. Live-cell imaging of activated $T$ cells pre-treated with Sema3AS-P-I-AF647 and allowed to form synapses on supported lipid bilayers with ICAM-1, CD80 and H-2Kb-SIINFEKL. Arrows in merged image indicate cells that have bound Sema3A and do not form immunological synapses. D. Representative image from high-throughput analysis of immunological synapses on supported lipid bilayers as in (C) with OT-I T cells pre-treated with Sema3A or not. E. Quantification of immunological synapses with or without Sema3AS-P-I pre-treated OT-I T cells. Data from six independent experiments $(n=90-1100$ cells per mouse per group). ${ }^{\star *}=P<0.01$, by paired t-test. Abbreviations: IRM, interference reflection microscopy. Sec, seconds. 


\section{Sema3A affects $\mathrm{T}$ cell actin dynamics through actomyosin II activity}

Class 3 semaphorins have been shown to have various effects on the cytoskeleton in hematopoietic cells, including thymocytes (27), dendritic cells (23) and T cells (12), however the precise nature of these effects in CD8+ T cells is not well characterized. Since cytoskeletal F-actin remodeling is necessary for T cell binding to target cells (28) as well as lamellopodium (24) and IS formation (29, 30), we examined F-actin content and dynamics in T cells during Sema3As-p exposure. We first treated stimulated OT-I T cells with Sema3As$P$ at varying durations and examined F-actin content using flow cytometry. Surprisingly, no actin depolymerization was observed up to 30 minutes after Sema3As-p treatment (Figure 4A). To better visualize F-actin dynamics before and after Sema3As-p treatment, we crossed LifeAct-eGFR (31) mice with OT-I mice to generate LifeAct-OT-I T cells. Mice developed normally and generated Ova-specific T cells with GFP-labelled F-actin. Stimulated T cells formed an active lamellopodium that undulated across an activating surface containing CD3 and ICAM-1, allowing for close inspection of F-actin dynamics using time-lapse confocal microscopy. When Sema3As-p was added to cells during this undulating phase, $\mathrm{T}$ cell morphology changed and took a more irregular and roughened appearance (Figure 4B). During this phase, F-actin content at the surface interface did not change, but lamellopodia formation stopped and F-actin became non-dynamic and immobile (Figure 4C, 4F, Movie S4). We therefore analyzed F-actin velocity along the cell edge using kymographs (Figure 4D). Sema3as-p profoundly inhibited F-actin dynamics (mean velocity was $1.34 \mu \mathrm{m} / \mathrm{min}$ after treatment versus $3.8 \mu \mathrm{m} / \mathrm{min}$ before) (Figure 4E). Next, we treated T cells with mutant Sema3A and found no difference in F-actin dynamics after treatment (Figure 4E), confirming that the effect of Sema3A on F-actin in the lamellopodia is NRP1-dependent. We assessed if this stark effect was due to localized F-actin depolymerization at the interface. Consistent with our flow cytometric analysis of global F-actin abundance (Figure 4A) however, the fluorescence intensity of LifeAct at the interface did not change, although the F-actin network contracted, and the cell width shrank substantially following treatment with Sema3As-P (Figure 4F-G). Because these effects on the actin cytoskeleton suggested that F-actin turnover dynamics could be affected, we tested wheather Jasplakinolide treatment would phenocopy the effects of Sema3As-PI. However, this instead led to constant shrinking of the cells' F-actin network, not the immobilizing effects Sema3A produced (Figure 4G). 
222 Sema3A signaling through Plexin-A1 inactivates the small GTPase Rap1A (32), which in 223 turn modulates myosin-IIA activity in diverse cell types $(33,34)$. The effects on the $T$ cell 224 cytoskeleton we observed in the presence of Sema3As-p appeared consistent with increased 225 myosin-IIA activity. We therefore visualized and quantified the contact area of undulating T 226 cells before and after Sema3As-p treatment followed by treatment of the myosin-II inhibitor 227 Blebbistatin. As the border of IRM and F-actin signal overlay completely (Supplementary 228 Figure 3F), we quantified IRM area to avoid phototoxic effects and inactivation of 229 Blebbistatin, which would be caused by exciting LifeAct (35). When Sema3A was added, T 230 cell contact area contracted significantly and cells became immobilized, in line with our 231 analysis of F-actin (Figure 4F-G). However, when Blebbistatin was added, T cells started 232 undulating and regained their former size (Figure $\mathbf{4 H}$, Movie S5). Conversely, when cells 233 were pre-treated with Blebbistatin followed by Sema3As-P, they retained their shape and 234 activity (Figure 4I, Movie S6). We therefore conclude that Sema3A inhibits F-actin dynamics 235 in CD8+ T cells, through hyper-activation of myosin-IIA. 
A
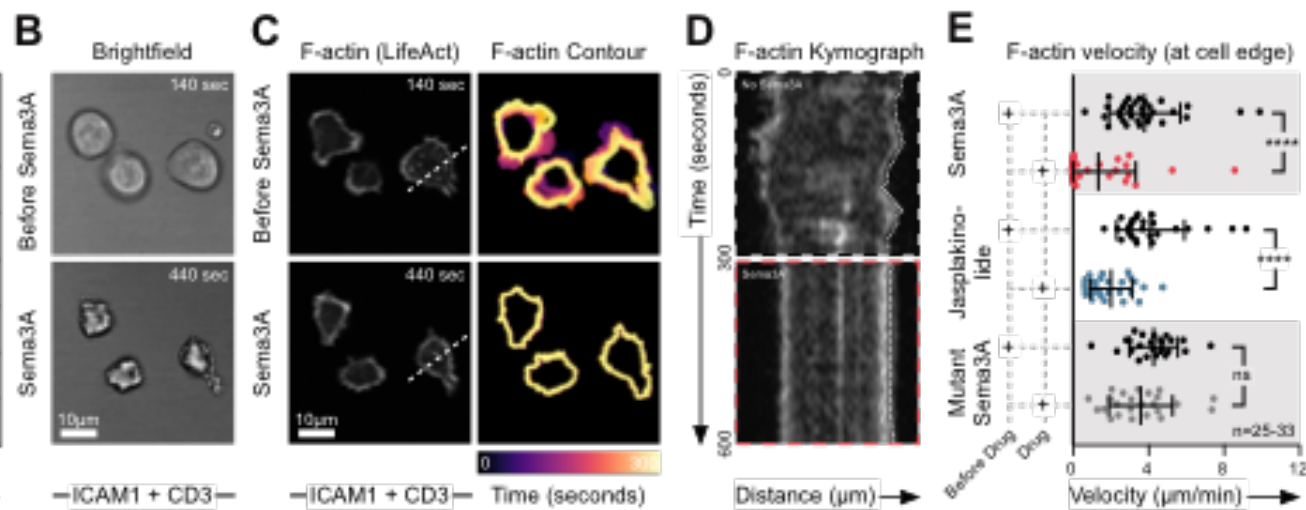

$\mathbf{F}$

Lifeact Intensity Plot - Before Bemasa

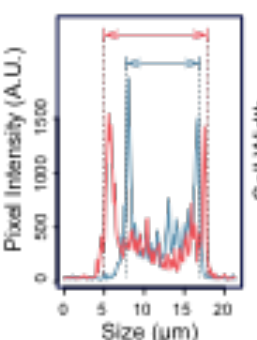

Cell Width - Befare Semaja - vater semina

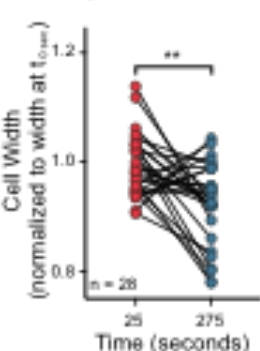

G

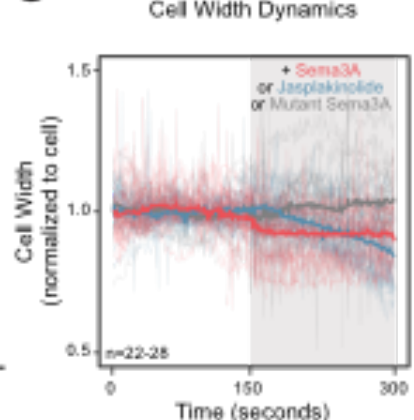

H
H
I

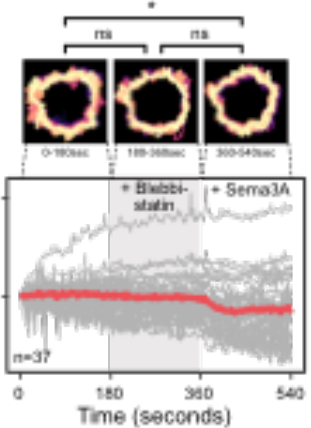

Figure 4. Sema3A affects T cell actin dynamics through actomyosin II activity.

A. Representative flow cylometric analysis of F-actin content with no or varying exposure to Sema3AS-P treatment in 48 hour stimulated OT-1 T cells as measured by Phalloidin-staining. Percentage indicate posittve cells in each condition. Data representative of two independent experiments. B. Representative brightfield images of 48 hour stimulated LAeAct OT-I T cells adhering to ICAM-1 and CD3 coated plates betore and after Sema3AS.P added to medium. C. Representative contocal images of LifeAct in OT-I T cells (left) and their contour plots (right) from same experiment as in (B), Image taken at cell-surface interface. Dashed white line indicate area used for (D). Color of contour indicates time from 0 to 300 sec as denoted on scalebar. D. Kymograph before (top) and after (bottom) Sema3AS-P added to medium on area indicated with whilte dashed line in (C). Dotted line along edge of cell denoted example of data used for calculating data in (E). $E$. Quantification of F-actin velocity at cell edge before and after treatment with either Sema3AS-P. Jasplakinolide or mutant Sema3A (in=25-33 cells per group) using same experimental setup as in (B). Data combined from three independent experiments and indicate mean \pm SD. ${ }^{*+\cdots}=\mathrm{P}<0.0001$, ns $=$ not significant, by paired $\mathrm{t}$ test. F. Intensity plot of LiteAct signal before and after Sema3AS-P treatment of a single OT-I T cell (left) or quantied on mutiple cells exposed to Sema3AS-P (right) using same experimental setup as in (B). Arrows indicate measured cell width. " $=\mathrm{P}<0.01$, by paired t-test $\mathrm{G}$. Cell width dynamics measured like $\langle\mathrm{F}\rangle$ over time betore (white background) or atter (grey background) Sema3AS-P. Jasplakinolide or mutant Sema3A addition to medium. $\mathbf{H}$. Cuantification of IRM area of individual OT-I T cells (grey lines) $\propto$ average for group (red line) ower time, with no treatment (leftmost while background). under treatment with Sema3A (grey background) and then Blebbistatin (rightmost white background). Above representative contour plots of single cell under different treatments, with color denoting time (150 sec total). Cells were allowed to settile, and form contact for $3-5 \mathrm{~min}$ betore data acquisinion. Area normalized to cell area at $\mathrm{t}=0$ sec. Data combined from three independent experiments ( $\mathrm{n}=27 \mathrm{cells}$ ). $\cdots=\mathrm{P}<0.001, \cdots=\mathrm{P}<0.0001, \mathrm{~ns}=$ not significant by two-way ANOVA at time-points 90,270 and 450 sec. $\mathrm{t}$ Quantificantion of IRM area of individual OT-I T cells and representative contour plots as in $(\mathrm{H}$ ), but with treatment with Blebbistatin (grey background) before Sema3AS-P (rightmost white background). Data combined from three independent experiments $(n=37$ cells). " $=P<0.05$, ns $=$ not significant by two-way ANOVA at

\section{Nrp1-deficiency enhances anti-tumor activity of CD8+ T cells against Sema3A-rich} tumors

241 To investigate the functional importance of Sema3A in suppressing T cell migration and IS 242 formation in vivo, we pursued two complementary lines of enquiry. During development, 243 CD8+ T cells express CD4 molecules during a CD4-CD8 double positive stage (36). Thus 244 we crossed LoxP-flanked (Flox) Nrp1 mice with CD4-Cre mice to generate CD4-Cre X $245 \mathrm{Nrp1}^{+/+}$, CD4-Cre $\mathrm{X} \mathrm{Nrp} 1^{\mathrm{Flox} /+}$ and CD4-Cre $\mathrm{X}$ Nrp1Flox/Flox mice (hereafter referred to as $246 \mathrm{Nrp1}^{+/+}, \mathrm{Nrp} 1^{\mathrm{Flox} /+}$ and Nrp1 Flox/Flox, respectively), to generate Nrp1-deficient $\mathrm{T}$ cells. 247 Disruption of Nrp1 expression on stimulated CD8+ T cells was confirmed by flow cytometric 
analysis (Supplementary Figure 4A), thereby generating mice with $\mathrm{T}$ cells insensitive to Sema3A ligation. Mice bred normally, had no gross anatomical differences, grew at similar rates and showed no sign of splenomegaly (Supplementary Figure 4B-C). Analysis of thymocyte subsets and differentiated $\mathrm{T}$ cell memory subsets in the spleen revealed no differences between genotypes (Supplementary Figure 4D-E), suggesting that NRP1 is not involved in thymocyte development or T cell homeostasis in non-inflamed conditions. CD8+ T cells from mice of all genotypes expressed similar levels of effector cytokines following CD3/CD28 stimulation (Supplementary Figure 4F). We next set out to establish the role of NRP1 on CD8+ T cell priming and activation by infecting mice with the A/PR/8/34derived pseudotyped influenza virus H7 (Netherlands/2003) N1 (England/2009) (here called $\mathrm{H} 7 \mathrm{~N} 1 \mathrm{~S}-\mathrm{Flu})$. This virus is capable of triggering strong $\mathrm{H}-2 \mathrm{D}^{\mathrm{b}}$-restricted influenza nucleoprotein (NP)-specific CD8+ $\mathrm{T}$ cell responses, but due to suppression of the hemagglutinin (HA) signal sequence cannot replicate or generate anti-HA specific neutralizing antibodies (37). This allowed us to specifically consider T cell responses. Mice were infected intranasally with H7N1 S-flu and weighed daily. No differences in weight between genotypes was observed (Supplementary Figure 4G). We detected no differences in percentage or absolute number of $\mathrm{H}-2 \mathrm{D}^{\mathrm{b}}$ NP-tetramer positive CD8+ T cells in lungs, draining lymph nodes (dLN) or spleen, ten days post-infection (Supplementary Figure 4H-I). Examining the phenotype of CD8+ T cells in the lung, we found that infected mice from all genotypes had an expansion of effector T cells as compared to uninfected mice (Supplementary Figure 4J). Consequently, we conclude that NRP1 is dispensable for CD8+ T-cell priming and activation.

We then challenged Nrp1 ${ }^{+/+}$, Nrp1 $1^{\text {Flox/+ }}$ and Nrp1Flox/Flox mice with B16.F10 and Lewis lung carcinoma (LL/2) cells. The poor immunogenicity of both cell-lines has been overcome using combination therapies that augment immune responses, such as anti-PD1 and anti-4-1BB (38, 39), and we therefore considered them good models for examining $\mathrm{T}$ cell anti-tumor activity. Notably, Nrp1Flox/Flox mice had significantly delayed tumor growth and increased survival when challenged with either B16.F10 or LL/2 (Figure 5A-B, Supplementary Figure 4K). We confirmed that this effect was dependent on CD8+ T cells, as antibody-mediated depletion of CD8+ T cells allowed B16.F10 cells to grow unperturbed in Nrp1Flox/Flox mice 
(Figure 5C, Supplementary Figure 4L). When examining levels of tumor-infiltrating lymphocytes (TILs) in Nrp1+/+, Nrp1 $1^{\mathrm{Flox} /+}$ and Nrp1 $1^{\mathrm{Flox} / \mathrm{Flox}}$ mice, we noticed a significant increase in the numbers of CD8+ T cells within tumors in Nrp1Flox/Flox mice, but not of CD4+ $\mathrm{T}$ cells (Figure 5D, Supplementary Figure 4M). Bone-marrow (BM) chimeric mice, containing mixed Nrp1Flox/+ and Nrp1 $1^{\text {Flox/Flox }} \mathrm{BM}$, confirmed that the increased levels of infiltration were intrinsic to CD8+ T cells themselves and not dependent on unspecific effects by other cell types (Figure 5E).

We hypothesized that the reason $\mathrm{T}$ cell immunity was enhanced by NRP1-deficiency in our tumor models, but not against H7N1 S-flu, was an increased availability of Sema3A in the former. Indeed, we did not find high levels of Sema3A on either epithelial cells, leukocytes or endothelial cell-subsets in the lung before, during or after infection with H7N1 S-flu (Supplementary Figure 5A-B). Conversely, aggressively growing tumors such as B16.F10, often generate a hypoxic TME (40) which itself can induce Sema3A production (22). We cultured B16.F10 cells in normoxic or hypoxic conditions and performed RT-qPCR. As expected, hypoxic conditions led to upregulation of known hypoxic response genes, including Pdk1, Bnip3 and Vegfa, in addition to upregulation of Sema3A transcript (Supplementary Figure 5C). Flow cytometric analysis of B16.F10 cells grown for 11 days in vivo confirmed expression of Sema3A within the TME (Supplementary Figure 5D-E). In order to better control the level of Sema3A within the TME, we therefore generated B16.F10.Ova cells that either overexpress or lack Sema3A, upon gene disruption by CRISPR/Cas9 (here referred to as Sema3A OE and Sema3A KO, respectively). Deepsequencing, RT-qPCR for Sema3a transcript, and analysis by flow cytometry, confirmed that cells lacked or over-expressed Sema3A (Supplementary Figure 5F-H). Sema3A OE and Sema3A KO cell-lines grew at similar rates compared to wild-type B16.F10.Ova cells under both normal growth conditions and in the presence of the proinflammatory cytokines IFNY and TNFa in vitro (Supplementary Figure 5I). Importantly, when we injected Sema3A $\mathrm{OE}$ and $\mathrm{KO}$ cell lines into opposite flanks of C57BL/6 mice, tumors grew at similar rates (Figure 5F), thus confirming that the cell-lines had a comparable phenotype and growth potential. However, when we adoptively transferred stimulated OT-I T cells into these mice, 
310 (Figure 5G). These results demonstrate that Sema3A overexpression within the TME was 311 sufficient to effectively suppress tumor-specific killing. Furthermore, significantly fewer OT-I 312 T cells had infiltrated tumors that overexpressed Sema3A, compared to Sema3A KO tumors 313 (Figure $5 \mathbf{H}$ ). Taken together, our data underscores the functional significance of Sema3A 314 within the TME as a potent inhibitor of CD8+ T cell migration, and thereby anti-tumor 315 immunity, via interaction with NRP1. 
A
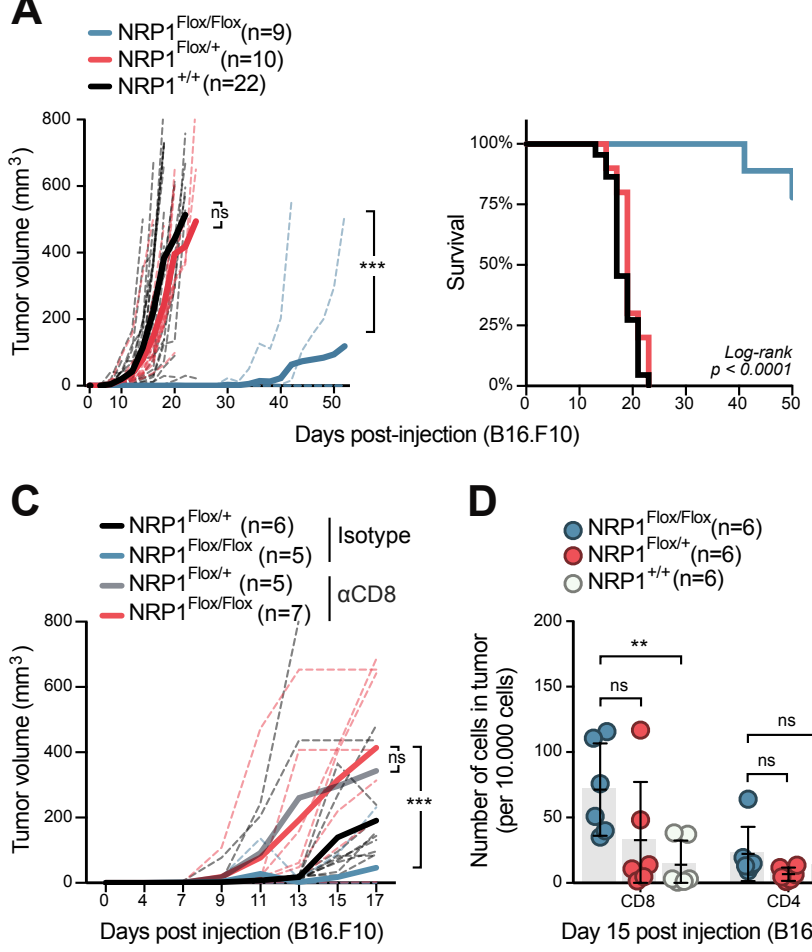

F

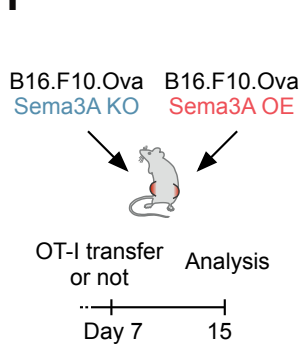

D $\mathrm{NRP}{ }^{\text {Flox/Flox }}(\mathrm{n}=6)$
$\mathrm{NRP} 1^{\text {Flox/+ }}(\mathrm{n}=6)$
$\mathrm{NRP}^{+/+}(\mathrm{n}=6)$

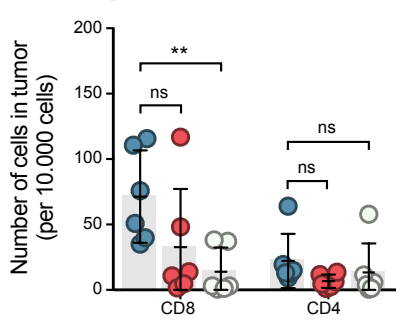

Day 15 post injection (B16.F10)
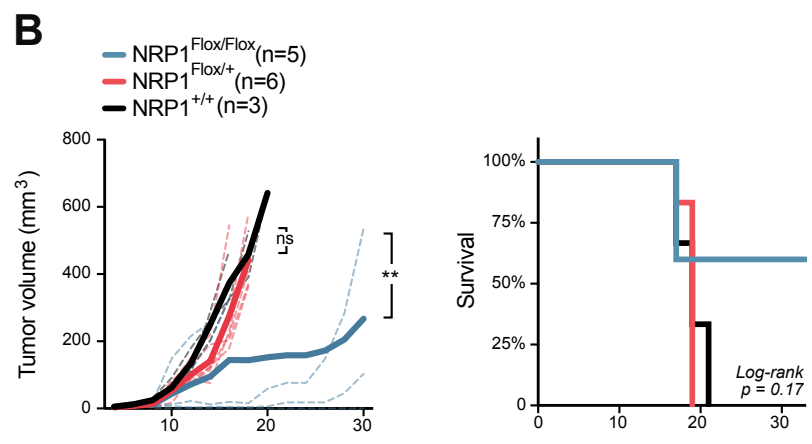

E
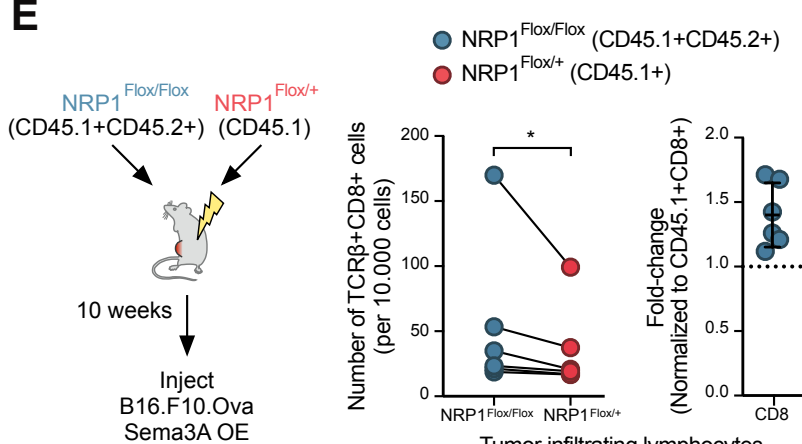

Tumor infiltrating lymphocytes
H
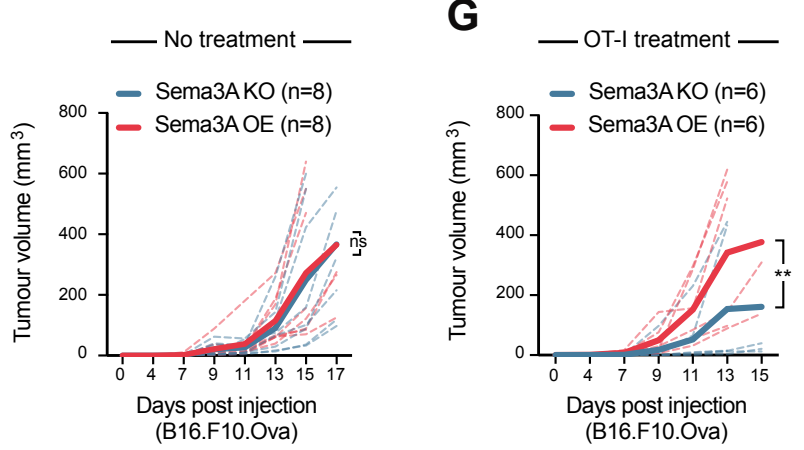

Figure 5: Nrp1-deficiency enhances anti-tumor migration and activity of CD8+ T cells.

A. Growth curve of B16.F10 cells in NRP1+/+, NRP1Flox/+ and NRP1Flox/Flox mice (left) and Kaplan-Meier survival curve (right). Dashed lines indicate growth in individual mice, bold line average for group. Combined data from 4 independent experiments with $3-6$ mice per group. ${ }^{\star \star \star}=P<0.001$, ns $=$ not significant by twoway ANOVA. B. Growth curve of LL/2 cells in NRP1+/+, NRP1Flox/+ and NRP1Flox/Flox mice (left) and Kaplan-Meier survival curve (right) ( $\mathrm{n}=3-6$ mice per group). Dashed lines indicate growth in individual mice, bold line average for group. Experiment performed once. ${ }^{\star \star \star}=\mathrm{P}<0.001$, ns $=$ not significant by two-way ANOVA. C. Growth curve of B16.F10 cells in NRP1Flox/+ and NRP1Flox/Flox mice pre-treated with either anti-CD8 antibody or isotype control ( $\mathrm{n}=5-7$ mice per group). Dashed lines indicate growth in individual mice, bold line average for group. Data combined from two independent experiments. ${ }^{\star \star \star}=P<0.001$, ns $=$ not significant by twoway ANOVA. D. Enumeration of CD4+ and CD8+ T cells infiltrated into B16.F10 tumors in NRP1+/+, NRP1Flox/+ and NRP1Flox/Flox mice ( $\mathrm{n}=6$ per group). Data indicate mean $\pm \mathrm{SD}$. ${ }^{* *}=\mathrm{P}<0.001, \mathrm{~ns}=$ not significant by two-way ANOVA. E. Experimental setup of mixed bone-marrow chimeras in C57BL/6 mice (left) and subsequent enumeration of CD8+ T cells in mice (middle graph). Ratio of CD8+ T cells from NRP1Flox/Flox to NRP1Flox/+ bone-marrow derived cells (right graph) $\left(n=6\right.$ mice per group). Experiment performed once. Data indicate mean \pm SD. ${ }^{*}=P<0.05$ by one-way ANOVA. F. Experimental setup using B16.F10 Sema3A KO or Sema3A OE cells (left) and growth curve of cells in untreated mice (right) ( $n=8$ mice). Experiment performed once. $n s=$ not significant by two-way ANOVA. G. Growth curve of B16.F10 Sema3A KO or Sema3A OE cells using similar experimental setup as in (F), but with OT-I treatment at day 7 post-injection ( $\mathrm{n}=6$ ). Experiment performed once. ${ }^{* *}=\mathrm{P}<0.001$ by two-way ANOVA. H. Enumeration of OT-I T cells in tumors (left graph) and their ratio of cells, normalized to the

NRP1 is expressed on tumor-infiltrating CD8+ T cells in clear cell renal cell carcinoma patients

321 We wished to explore if our findings were relevant to human cancer. Analysis of publicly 
survival in clear cell renal cell carcinoma (ccRCC) (Figure 6A). We hence turned to a cohort of ccRCC patients that had undergone nephrectomy (Supplementary Table 1) to explore the role of the Sema3A/NRP1 pathway in cancer immunity (Figure 6B). We first quantified NRP1 expression on CD8+ T cells from peripheral blood (PBMCs), and CD8+ TILs within tumor and tumor-adjacent tissue. Significantly more CD8+ T cells in both tumor and tumoradjacent tissue expressed NRP1 (Figure 6C-D, Supplementary Figure 6A), suggesting that these cells would be sensitive to Sema3A. In our murine model, NRP1 expression correlated with antigen exposure (Figure 1C), and we therefore speculated that NRP1positive CD8+ TILs might be tumor-specific. Indeed, most NRP1+ TILs were also PD1positive (Figure 6E-F), demonstrating that they had either recently been activated or experienced chronic exposure to antigen (41). To investigate this, we single-cell sorted NRP1-negative and positive CD8+ T cells from four ccRCC patients and examined their TCR repertoire. TCR diversity, as calculated by either Shannon (SA) and Simpson (SI) diversity indices of CDR3 $\beta$ (Figure 6G) and TRBV usage (Figure 6H), showed that NRP1+ CD8+ TILs were more clonal than NRP1-T cells, further supporting the hypothesis that such T cells had undergone clonal expansion following recognition of their cognate antigens (42)

(Figure 6E-F). Various cancer-testis (CT) antigens can be expressed by cancerous tissue in ccRCC (43). We took advantage of this fact and screened four HLA-A2-positive patients for the presence of HLA-A2-restricted CT-antigen-specific CD8+ T cells using a panel of 21 HLA-A2 tetramers loaded with CT epitopes (44). In the three patients, who had CT tetramer positive TILs, we found that a larger proportion of NRP1+ CD8+ TILs were specific for CTantigens (Figure 6I-J, Supplementary Figure 6B). Taken together these data show that NRP1+ CD8+ TILs were found in CCRCC patients, were activated, and were likely specific for tumor-associated antigens.

We next wished to explore the spatial distribution of Sema3A and CD8+ T cells within the TME. For this purpose, we stained ccRCC tissue sections from 12 patients from our ccRCC cohort for Sema3A by immunohistochemistry (IHC). We observed widespread expression of Sema3A both within the tumor as well as in adjacent non-neoplastic kidney tissue. In the tumor, Sema3A was predominantly expressed by smooth muscle cells within the tunica media of tumor vasculature but also in areas of fibromuscular stroma. In the adjacent tissue, 
354 glomerular mesangial cells and smooth muscle cells within peritubular capillaries stained 355 positive for Sema3A (Supplementary Figure 6C). Next, strict serial sections from the same 356 formalin-fixed paraffin embedded tissue blocks were stained for CD31 and CD8 and 357 computationally aligned to the Sema3A sections. Pathological review confirmed that 358 expression of Sema3A co-localized with that of the blood vessel marker CD31. Furthermore, 359 CD8+ cells were often located within regions of high Sema3A expression (Supplementary 360 Figure 6D); indeed dual-staining of CD31 and CD8 in CCRCC clearly showed that CD8+ 361 cells were restricted to the immediate area surrounding blood vessels (Figure 6K). To 362 further explore the effect of Sema3A on CD8+ cell infiltration and localization, we compared 363 regions within each tumor that were either Sema3A-rich or Sema3A-poor, allowing us to 364 control for variability in CD8+ cell infiltration between patients (Figure 6L). This analysis 365 confirmed that our selected Sema3A-rich regions expressed more CD31 than the Sema3A366 poor regions, underscoring the close association of Sema3A with the vasculature 367 (Supplementary Figure 6E-F). In 11 out of 12 examined patients, there were significantly 368 more CD8+ TILs in the Sema3A-rich areas than in Sema3A-poor areas, corresponding to 369 46\% fewer CD8+ cells in Sema3A-poor regions (Figure 6M). Additionally, the CD8+ cells 370 that were present in Sema3A-poor regions were often found clustered near sources of 371 Sema3A (Figure 6L, arrows). The data presented here are consistent with a role for 372 Sema3A in modulating $T$ cell infiltration and restricting CD8+ cells to perivascular areas 373 within the tumor. 
bioRxiv preprint doi: https://doi.org/10.1101/849083; this version posted November 20, 2019. The copyright holder for this preprint (which was not certified by peer review) is the author/funder. All rights reserved. No reuse allowed without permission.

A

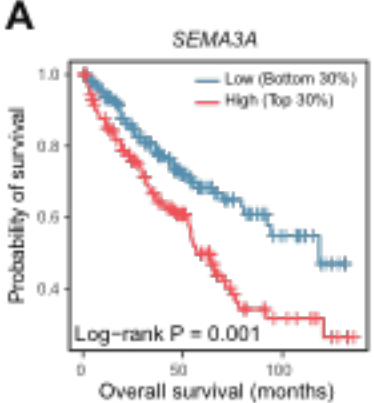

E

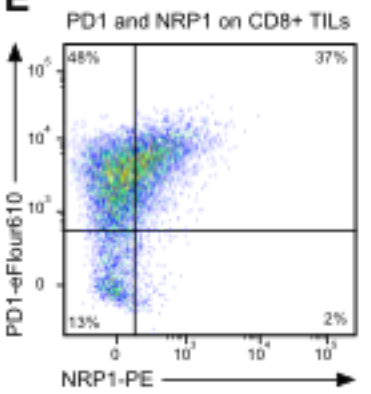

I

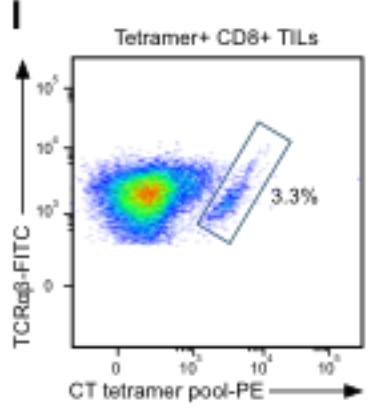

K

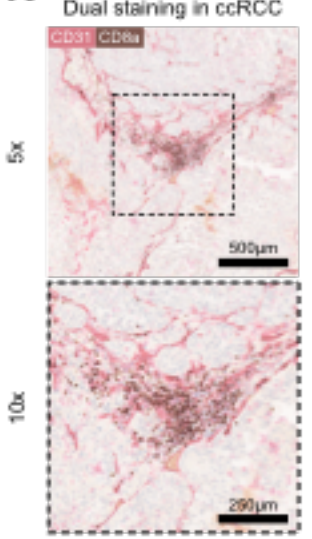

B

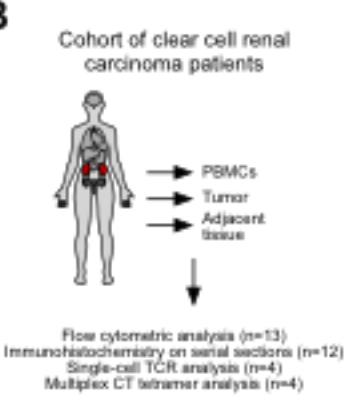

$\mathbf{F}$

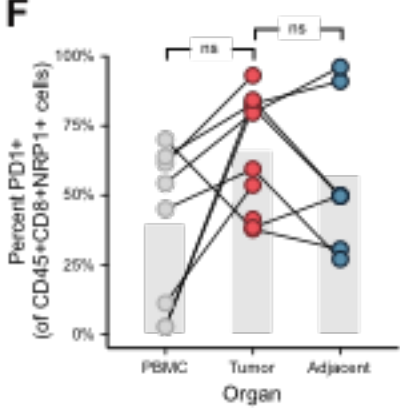

G

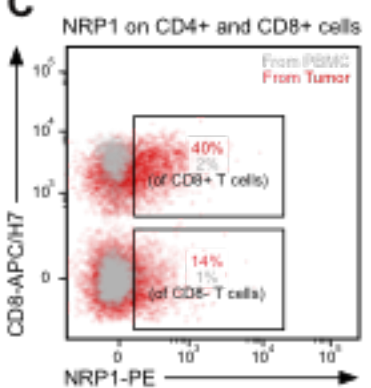

CDR3B diversity in CO8+ TIL.8

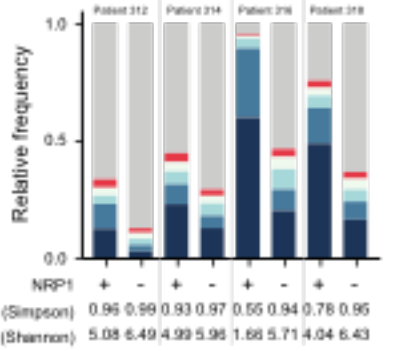

$J$
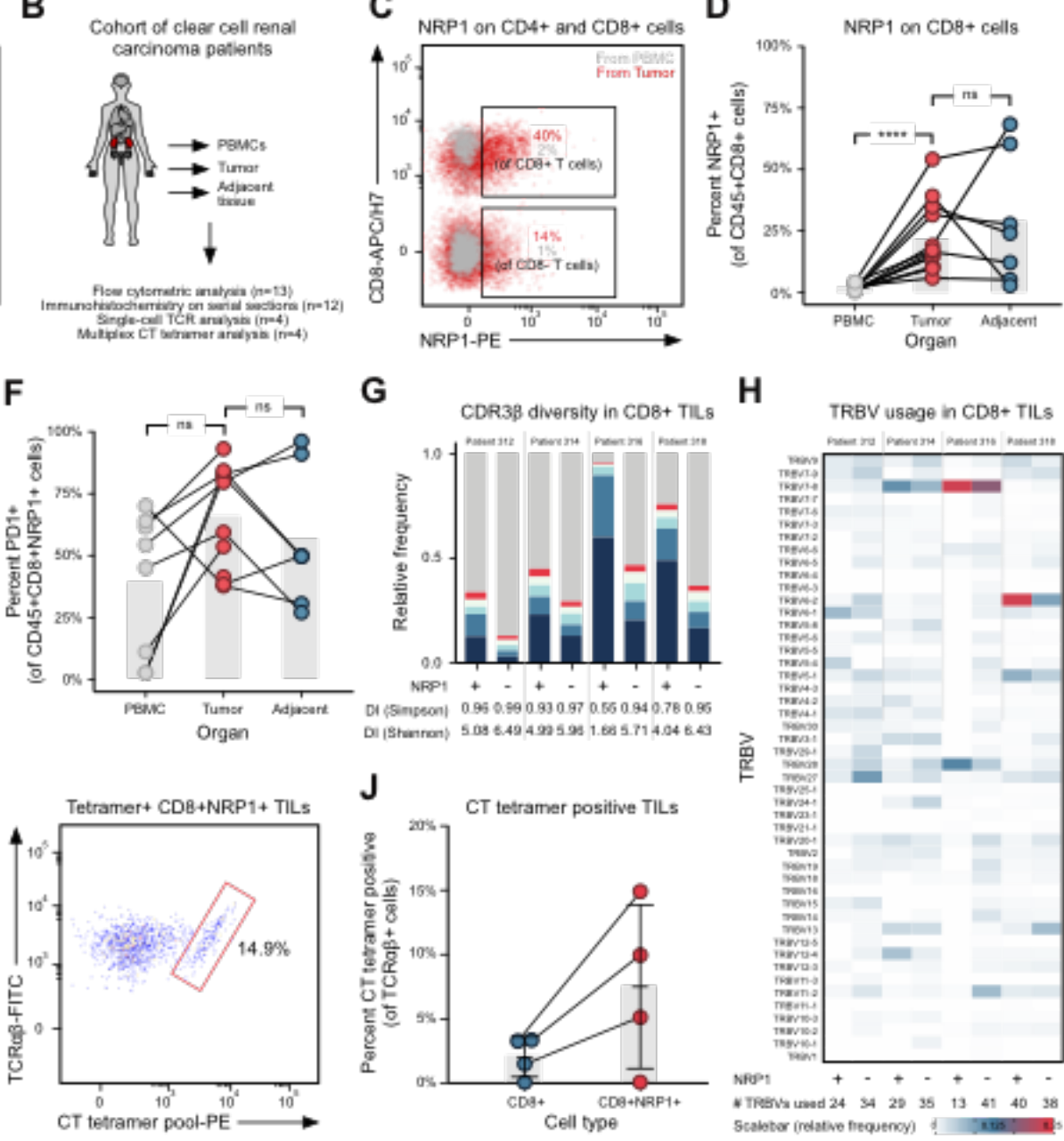

H

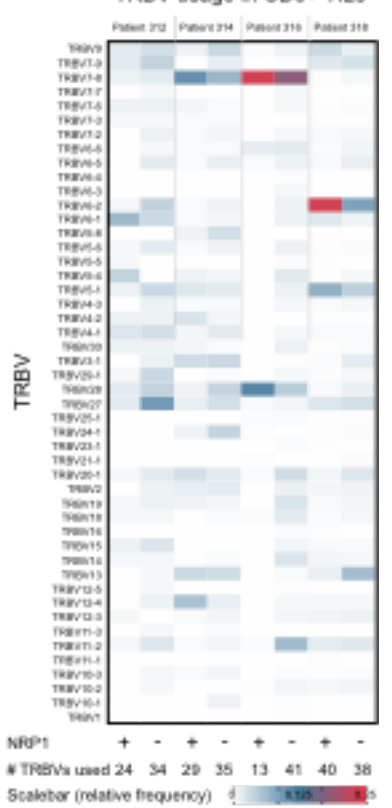

L $\quad \cos 1$

Sema3A

CO8

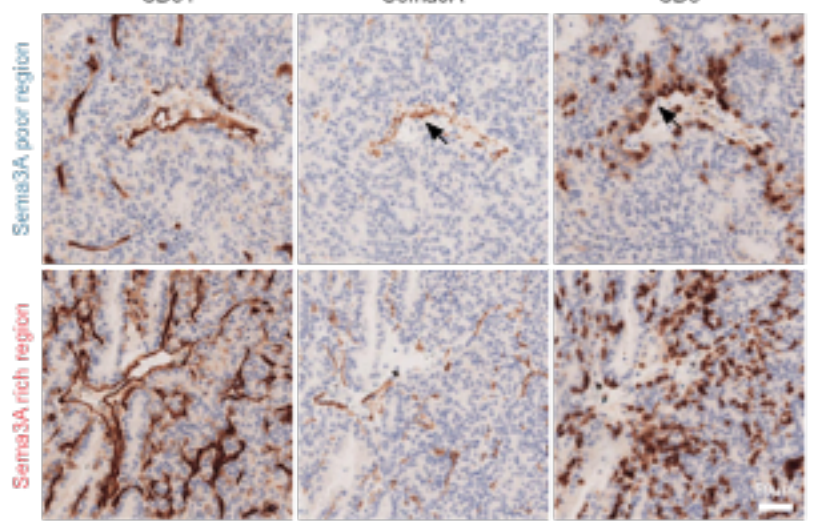

M
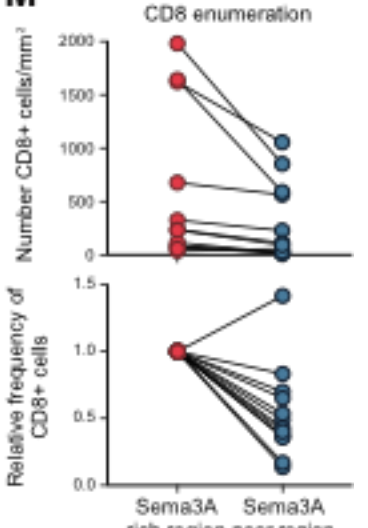

Figure 6: CD8+ TLLs express NRP1 and are captured in Sema3A rich areas in ccRCC tumors.

A. Correlation of SEMA3A mRNA level with survival of $x$ ACC patiants. Data from TCGA using TIMER (71). B. Schematic representing $\propto$ ACC cohort of patient utilized in [C-M) C. Pepresentative flow cytometric analysis of CD8 and NPP1 expression in PBMC and TI-s in cc.PCC patient D. Analysis of NPP1 expression

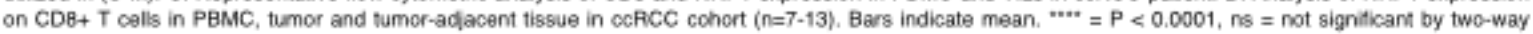
ANOVA, E. Representative flow cylometric analysis of PD1 and NAP1 on CD8+ TIL in ccRCC patient. F. Analysis of PD1 expression on NRP1 positive CD8+ T cells in PBMC, fumor and tumor-adjacent tissue in $\propto$ CACC cohort (n=7-13). Bars indicate mean. ns = not significant by two-way ANOVA. $\mathrm{Q}$. Analysis of COR3B diversity in NRP1 positive (+) and negative (-) CD8+ TILs (n=4), Colored bars represent the the most abundant clonotypes. Grey ber represents remaining sequences. SI and SA diversity indices (DI) show that in all four patients, NFP1 + TILs are less diverge. H. Heatmap of TRBV usage in NAP1 positive (+) and negative (-) CD8 $+\mathrm{TL}-\mathrm{s}(\mathrm{n}=4)$. Color indicates relathe usage within all of individual patients, as indicated by scalebar. $\mathrm{I}$. Fepresentative flowr cremetric analysis of TCRaß and CT tetramer positive CD8+ TILs (left) and NRP1+ CD8+ TILs (right). Error bars indicate mean 2 SD. J. Graph of percentage CT tetramer positive NAP1+ (red) and NRP1- (blue) CD8+ TILs in four ccRCC patients. K. Representative CD8 (brown) and CD31 (red) staining in cchCC tumor. Dashed area in top image indicates zoom area in bottom image. Scalebar, 500um and 250um. L. Representative CD31, Sema3A and CD8 staining in Sema3A poor region (top row) and Sema3A rich region (bottom row). Arrows indicate association between Sema3,A and CD8 staining. Scaleber, 50 um. M. Enumeration of CD8+ TILs in Sema3A rich (red dots) and poor (blue dots) in patients ( $n=12)$. Abbreviations: CT, cancer testis. DI, divergity indices. SA, Shannon index. SI, Simpson index. TIL,

fumor-infiltrating lymphocytes. TCGA, The Cancer Genome Atlas. TIMER, Tumor Immune Estimation Resource. TRBV, TCR beta chain variable. 


\section{DISCUSSION}

\section{8}

In this study we showed that the secreted protein Sema3A has a previously underappreciated role in controlling tumor-specific CD8+ $T$ cells and highlight several important conclusions concerning its function.

We established several lines of evidence that reveal a strong inhibitory effect of Sema3A on 383 384 385 386 CD8+ $T$ cell migration in tumors. First, in vitro experiments provided functional insights into how Sema3A inhibited key steps in T cell extravasation, including adhesion, transmigration and mobility. Notably, these effects could be reversed using a blocking antibody against NRP1, confirming that NRP1 is an important regulator of Sema3A signaling on CD8+ T cells. Second, conditional knockout of NRP1 on T cells corroborated these findings in vivo, resulting in higher CD8+ $T$ cell infiltration into the TME. Conversely, significantly fewer tumor-specific $T$ cells homed to and infiltrated Sema3A-overexpressing tumors. Third, in cCRCC patients CD8+ TILs were preferentially found in Sema3A-rich regions and beside Sema3A-rich blood vessels, reminiscent of how tumor-associated macrophages can be entrapped within Sema3A-rich hypoxic regions (22).

We explored the effect of Sema3A on IS formation. Previous studies have characterized Sema3A as an inhibitor of $T$ cell signaling and proliferation using in vitro assays $(8,12)$. We extended these results and confirmed that key steps in synapse formation are affected, including cell-cell binding, formation of close contact zones and organization of distinct supramolecular activation clusters. These findings are in line with work by Ueda et al who found that Sema3E inhibited IS formation in thymocytes (27). We further show that the Factin cytoskeleton becomes activated following Sema3A exposure. Although further experiments are warranted to draw firm conclusions, this effect is ostensibly dependent on myosin-IIA activity, since we could rescue T cell undulation using the drug Blebbistatin which specifically prevents intra-cellular force generation by myosin-II (45). High resolution 3D imaging has shown that myosin-IIA forms bona fide arcs above the pSMAC $(46,47)$ but then moves inwards and contracts, thereby pinching the T cell away during termination of the IS 
(24). This isotropic contraction of the actomyosin arc appears similar to myosin's role during cytokinesis (48). Our data suggest that Sema3A leads to hyperactivation of myosin-II, thus enforcing IS termination. Data does exist to provide a link between Sema3A binding and myosin-II. Biochemical and crystallographic studies have shown that Sema3A signaling converts the small GTPase Rap1A from its active GTP-bound state, to its inactive GDPbound state following binding to Plexin-A $(15,32)$. In epithelial and endothelial cells, active Rap1-GTP can act as a negative regulator of myosin-II (33). It is therefore tempting to speculate that Sema3A, by inhibiting Rap1-GTP activity, leads to hyperactivation of myosinII. Indeed, in both neurons $(7,49)$ and DCs $(23)$, Sema3A has been shown to increase myosin-II activity in line with this interpretation, however much of this pathway needs to be further elucidated in T cells. We propose that Sema3A induces a cellular "paralysis" based on integrin-actinomyosin contraction leading to motility paralysis and immunological synapse preemption.

There is a growing interest in NRP1 in the context of T cell anti-tumor immunity. Much research has focused on regulatory T cells (Treg), as NRP1 can be used to identify thymusderived regulatory $\mathrm{T}$ cells $(50)$ and has been shown to play an important role in controlling Treg function and survival (51). It has become clear that NRP1 is expressed on dysfunctional tumor-specific CD8+ T cells (9-11), indicating that the protein might play an important role in regulating CD8+ T cells as well. We show that initial NRP1 expression is controlled by the level of TCR-engagement, that the protein remains expressed on tumor-specific CD8+ T cells in vivo and that NRP1 is found on a subset of tumor-specific CD8+ T cells in human ccRCC patients. These latter results are in line with reports by Jackson et al. (52) and Leclerc et al. (11) who found that approximately $10 \%$ of CD8+ TILs from melanoma patients and $14 \%$ of non-small-cell lung cancer patients, respectively, were NRP1 positive. Unlike us, Jackson et al. did not find any role for NRP1 in regulating CD8+ T cells when mice were challenged with a leukemia cell line. An explanation for this discrepancy could be a lack of Sema3A expression in this model. Indeed, we did not find any functional differences between NRP1 knockout and wild-type T cells when challenging mice with H7N1 S-Flu, a pathogen that did not lead to any meaningful upregulation of Sema3A in the lung. 
significant effects on $\mathrm{T}$ cell migration and control of tumor growth when treated with tumorspecific CD8+ T cells. An even stronger effect was seen in the lack of tumor growth in our Nrp1Flox/Flox mice. These results are in line with Delgoffe et al. (51) and Leclerc et al. (11) who show similar control of tumor growth when treating mice with a blocking anti-NRP1 antibody. Hansen et al. also found strong anti-tumor effects in a comparable conditional NRP1 knockout model but ascribed this to decreased Treg infiltration into the TME (53). More likely, the remarkable control of tumor growth seen by us and others is due to synergistic effects, as our data would suggest that ablation of NRP1 enhances CD8+ T cell migration and effector functions as well. Research by Vignali and colleagues has shown that NRP1 plays a key role in Treg survival and suppressive capabilities within the TME through ligation with Sema4A $(51,54)$. Why does NRP1 enhance Treg function, but inhibit CD8+ T cells? While not exploring this question in detail, we did find that Tregs to a larger extent expressed other NRP1 co-receptors, including TGF $\beta R I$ and II. Indeed, NRP1 has been shown to enhance TGF $\beta$ binding in Tregs (18). As Tregs are dependent on TGF $\beta$ for their function (55), one intriguing possibility is that NRP1 preferentially partners with these TGF $\beta$ receptors on Tregs, while the only co-receptors available on CD8+ T cells are the proteins of the Plexin-A family, which could provide a molecular basis for distinct signaling in each cell type.

Our study highlights an underappreciated tumor-escape mechanism, namely inhibition of tumor-specific T cells through cytoskeletal paralysis. We find that the effects of Sema3A on CD8+ $\mathrm{T}$ cells are mainly mediated through the co-receptor NRP1, suggesting new therapeutic avenues, for example by using antagonistic NRP1 antibodies. Enhancing migration of tumor-specific $T$ cells into tumors is critical for improving the efficacy of checkpoint blockade (56) and adoptive T cell transfer therapies (57), making this an exciting prospect. However, since the Sema3A-Plexin-A-NRP1 pathway also regulates the maturation of endothelial cells (15) emphasis on timing and drug-target will be critical. 


\section{METHODS}

465

466

467

468

469

470

471

472

473

474

475

476

477

478

479

480

481

482

483

484

485

486

487

488

489

490

491

492

493

494

\section{Cell lines and media}

Cell culture was performed using antiseptic techniques in HEPA filtered culture cabinets. Cell lines were grown at $37 \mathrm{c}$ in a $5 \% \mathrm{CO}_{2}$ atmosphere. As indicated in text, for some experiments, cells were cultured for 24 hours in a $1 \% \mathrm{O}_{2}$ chamber. All cell-lines were screened for Mycoplasma. Adherent cells where split by Trypsin-EDTA detachment and serially passaged and their viability regularly checked.

B16.F10 and B16.F10.Ova cell-lines were provided by Uzi Gileadi. The latter was generated by transducing B16.F10 with a modified Ovalbumin construct, containing a start codon and amino-acid 47 to 386 of the full-length ovalbumin, which ensures that Ovalbumin is not secreted by the cell-line. LL/2 cells were a gift from Christopher W Pugh (Nuffield Department of Medicine, University of Oxford).

B16.F10.Ova Sema3A knockout cells were generated using CRISPR/Cas9 genome-editing (see below). B16.F10.Ova Sema3A overexpressing cells were generated by transducing cells with a lentivirus encoding EFS-Sema3A cDNA (NCBI sequence NM_001243072.1)mCherry, cloned by VectorBuilder (see below). HEK293T cells were a gift from Tudor A. Fulga (Radcliffe Department of Medicine, University of Oxford).

Adherent cells were kept in DMEM, 10\% FCS, 2 mM Glutamine, $1 \mathrm{mM}$ Sodium Pyruvate, $100 \mathrm{U} / \mathrm{ml}$ penicillin $+100 \mu \mathrm{g} / \mathrm{ml}$ streptomycin. For some experiments, $10 \mathrm{ng} / \mathrm{mL}$ murine IFNy (cat. no 315-05, PeproTech) or murine TNFa (cat. no. 315-01A, PeproTech) was added to medium. T cells were kept in IMDM, 10\% FCS, 2 mM Glutamine, 1 mM Sodium Pyruvate, 1x Non-essential amino acids, $100 \mathrm{U} / \mathrm{ml}$ penicillin $+100 \mu \mathrm{g} / \mathrm{ml}$ streptomycin, $10 \mathrm{mM} \mathrm{HEPES}$, $50 \mu \mathrm{M} \beta$-mercaptoethanol. 10 IU IL-2 (cat. no AF-212-12, PeproTech) was added from frozen stock just before use.

\section{Mouse strains and injection of tumor cells, T cells, antibodies and S-Flu H7N1}

All experiments were performed in mice on a C56BL/6 background. Mice were sex-matched and aged between 6 and 12 weeks at the time of the first experimental procedure. All studies were carried out in accordance with Animals (Scientific Procedures) Act 1986, and the University of Oxford Animal Welfare and Ethical review Body (AWERB) under project licence 
40/3636. CD4-Cre mice were a gift from Katja Simon (NDM, University of Oxford). LifeAct mice were a gift from Shankar Srinivas (DPAG, University of Oxford). C57BL/6, OT-I and CD45.1 mice were purchased from Biomedical services, University of Oxford. NRP1-floxed mice were purchased from Jackson Laboratories (Stock No: 005247).

Cancer cell lines were split at 1:3 ratio 24 hours before injection into mice in order to keep cells in log-phase. On the day of injection, cells where trypsinized and washed 3 times in PBS to remove residual FBS. Suspensions of $1.5 \times 10^{5}$ cells in $100 \mathrm{uL}$ PBS were prepared and kept on ice until injection. Mice were anesthetized using isoflurane and cells injected intradermally.

For adoptive transfer of T cells into mice, OT-I splenocytes were stimulated for 48 hours using SIINFEKL peptide and sorted as described below, washed 2 times in PBS and injected i.v. via the tail vein.

For infection with S-Flu H7N1, mice were infected intranasally with 10 infectious units S-Flu H7N1 in 50uL viral growth medium (DMEM with $2 \mathrm{mM}$ Glutamine, $10 \mathrm{mM}$ HEPES, $100 \mathrm{U} / \mathrm{ml}$ penicillin +100

$\mu \mathrm{g} / \mathrm{ml}$ streptomycin and $0.1 \% \mathrm{BSA}$ ) under anesthesia.

For CD8-depletion experiments, anti-CD8a (cat. no. BE0061, clone 2.43, BioXcell) or IgG2b isotype control (cat. no. BE0090, clone LTF-2, BioXcell) were resuspended in PBS and injected intraperitoneally at day $-4,-1,4$ and 7 post injection of cancer cells.

\section{Mixed bone-marrow chimeras}

To generate mixed bone marrow chimeric mice, male C57BL/6 host mice were lethally irradiated at 4.5 Gy for 300 seconds, followed by a 3 hour rest, and a subsequent 4.5 Gy dose for 300 seconds. Mice were injected i.v. with a 1:1 mixture of CD45.1+NRP1Flox/+ and CD45.1+CD45.2+ NRP1Flox/Flox bone marrow cells. Recipient mice received drinking water containing antibiotics $(0.16 \mathrm{mg} / \mathrm{mL}$ Enrofloxacin (Baytril), Bayer Coporation). Mice were rested for 10 weeks before experimental use.

\section{Analysis of publicly available transcriptional data}

For analysis of Sema3A co-receptors, we downloaded raw expression data collected from mice from the "Immunological Genome Project data Phase 1" via the Gene Expression 
Omnibus (series accession: GSE15907). We specifically focused on naïve CD8+ T cells (accessions: GSM605909, GSM605910, GSM605911), CD8+ effector T cells (accessions: GSM538386, GSM538387, GSM538388, GSM538392, GSM538393, GSM538394), and CD8+ memory T cells (accessions: GSM538403, GSM538404, GSM538405). The raw expression array files were processed using the affy package (58) and differential expression of selected genes (CD72, NRP1, NRP2, PLXNA1, PLXNA2, PLXNA3, PLXNA4, PLXNB1, PLXNB2, PLXNB3, PLXNC1, PLXND1, SEMA3A, SEMA3B, SEMA3C, SEMA3D, SEMA3E, SEMA3F, SEMA3G, SEMA4A, SEMA4B, SEMA3C, SEMA4D, SEMA4F, SEMA4G, SEMA5A, SEMA5B, SEMA6A, SEMA6B, SEMA6C, SEMA6D, SEMA7A, TIMD2, HPRT, OAZ1, RPS18, NFATC2, TBX21, EOMES, CD28, PDCD1, CTLA4, LAG3, BTLA, TIM3, ICOS, TNFRSF14, TNFSF14, CD160, CD80, LAIR1, CD244, CXCR1, CXCR2, CXCR3, CXCR4, CXCR5, CCR1, CCR2, CCR3, CCR4, CCR5, CCR5, CCR6, CCR7, CCR8, CCR9, CCR10) between naïve and effector and memory cells was examined using the limma package (59) in $\mathrm{R}(60)$.

Analysis of TCGA data was conducted using TIMER (61).

\section{Harvesting and activating splenocytes}

Mice were euthanized using $\mathrm{CO}_{2}$, and spleens were harvested and stored in T cell media on ice. The spleen was strained through a $70 \mu \mathrm{m}$ nylon mesh using the blunt end of a syringe to make a single cell suspension. Cells were washed off the mesh by applying $5 \mathrm{~mL}$ of $T$ cell medium, followed by mixing of the solution by aspiration. Cells were then washed and resuspended in $3 \mathrm{~mL}$ red blood-cell (RBC) lysis buffer for 5 minutes on ice. Cells were washed again in T cell medium, counted and resuspended at $2 \times 10^{6}$ cells per $\mathrm{mL}$ in $\mathrm{T}$ cell medium.

$10 \mathrm{IU} / \mathrm{ml}$ IL-2 (Cat. 212-12, PeproTech) and 25nM SIINFEKL (N4) peptide (Cambridge Peptides) were added to the single cell solution. Approximately $200 \mu \mathrm{L}$ cells were then plated onto a 96-well U-bottom plate and allowed to expand for 48 hours. For TCR affinity assays, SIINFEKL (N4), SIITFEKL (T4) or SIIQFEKL (Q4) peptide (Cambridge Peptides) was used at indicated concentrations.

\section{Sorting CD8+ T cells using magnetic beads}


CD8a+ Negative T Cell Isolation Kit (Order no. 130-104-075, Miltenyi Biotec) was used to sort T cells and was performed according to the manufacturer's protocol. Briefly, cells were washed in MACS buffer (0.5\% bovine serum albumin and 2 mM EDTA in PBS), incubated with antibody cocktail, followed by magnetic beads for 10 minutes each on ice. Cells were then loaded into a prewetted LS column (Order no. 130-042-401, Miltenyi Biotec) inserted into a magnet in approximately 3-5 $\mathrm{mL}$ MACS buffer.

\section{Preparation of tissue from mice for flow cytometry}

When staining cells in B16.F10 and LL/2 tumors, or from lymph nodes, frontal cortex, lungs or thymus, mice were euthanized using $\mathrm{CO} 2$, and tumors were harvested and stored in $\mathrm{T}$ cell media on ice. Organs were cut into smaller pieces with a scalpel and incubated for 30 minutes with reagents from a tumour dissociation kit (Order no. 130-096-730, Miltenyi Biotec). Cells were strained through a $70 \mu \mathrm{m}$ nylon mesh using the blunt end of a syringe to make a single cell suspension. Cells were washed off the mesh by applying $5 \mathrm{~mL}$ T cell media, followed by mixing of the solution by aspiration. After a wash, the cells were resuspended in approximately $2 \mathrm{~mL}$ of 100\% Percoll solution (Cat. no. 17-0891-01, GE Healthcare), and layered carefully on top of $3 \mathrm{~mL}$ of $80 \%$ and $40 \%$ Percoll solution, and spun for 30 minutes at $2000 \mathrm{~g}$. Cells at the $80-40 \%$ interphase were washed, and stained using protocols outlined below.

\section{Flow cytometry}

For washing and staining cells for flow cytometry PBS with 2\% BSA, $0.1 \%$ NaN3 sodium azide was used. Single colour controls were either cells or OneComp Compensation Beads (Cat. No 01-1111-41, Thermo Fisher).

For surface staining, cells were washed with $200 \mu \mathrm{l}$ FACS Buffer and blocked in $100 \mu \mathrm{Fc}$ block (cat. no. 101319, TruStain FCX, clone 93, BioLegend, diluted 1:100) in FACS Buffer for $10 \mathrm{~min}$ on ice and washed. Antibody cocktail was added and cells were stained on ice for at least $20 \mathrm{~min}$, in the dark and washed twice. When applicable, cells were fixed in $2 \%$ PFA for at least $10 \mathrm{~min}$ at RT before acquisition. For quantification of number of cells in tumors, lymph nodes and lungs in certain experiments, quantification beads (CountBright, cat. no. C36950, Thermo Fisher) were used. 
588 For intracellular staining, cells were fixed in $100 \mu \mathrm{l} /$ well of FoxP3 IC Perm/fix Buffer (Cat. no $589 \quad 00-8222-49$, Thermo Fisher) for $20 \mathrm{~min}$ at RT. Cells were pelleted, the fixative removed, and $590200 \mu \mathrm{l} /$ well of $1 \times$ Perm Buffer added for 20 min at RT and washed. Antibody mix was added 591 and cells stained for at least 20 min on ice in Perm buffer and washed twice.

592 Cells were analyzed on either Attune NxT (Life Technologies) or an LSR Fortessa X20 or 593 X50 (BD Biosciences) flow cytometers in the WIMM Flow Cytometry Facility and data was 594 analysed using FlowJo v10 (FlowJo) and R (60).

596 The following antibodies and tetramers were used for flow cytometry:

\begin{tabular}{|l|l|l|l|}
\hline Specific for & Fluorophore(s) & Company & Clone \\
\hline CD3e & BV650 & BioLegend & $17 A 2$ \\
\hline CD4 & APC710 & $\begin{array}{l}\text { Tonbo } \\
\text { Bioscience }\end{array}$ & GK1.5 \\
\hline CD4 & BUV810 & BD & GK1.5 \\
\hline CD8a & BV711 & BioLegend & $53-6.7$ \\
\hline CD11a & PE & BioLegend & M17/4 \\
\hline CD11b & APC & BioLegend & M1/70 \\
\hline CD19 & BV425 & BioLegend & $6 \mathrm{D} 5$ \\
\hline CD24 & APC710 & BD & M1/69 \\
\hline CD25 & PerCP/Cy5.5 & BioLegend & $3 \mathrm{C7}$ \\
\hline CD31 & BV510 & BD & MEC13.3 \\
\hline CD44 & APC/Cy7, & BioLegend & IM7 \\
\hline CD45.1 & PE/Cy7 & & \\
\hline CD45.2 & FITC & eBioscience & A20 \\
\hline CD49d & BUV395 & BD & BC10 \\
\hline CD62L & BV610,FITC & BioLegend & Mel-14 \\
\hline CD69 & BUV737 & BD & H1.2F3 \\
\hline CD103 & PE/Cy7 & BioLegend & 2 E7 \\
\hline
\end{tabular}




\begin{tabular}{|c|c|c|c|}
\hline CD105 & PE-CF594 & $\mathrm{BD}$ & MJ7/18 \\
\hline CD162 & BV421 & $\mathrm{BD}$ & 2PH1 \\
\hline EpCAM & APC/Cy7 & BioLegend & G8.8 \\
\hline $\mathrm{F} 4 / 80$ & BV610 & BioLegend & BM8 \\
\hline FoxP3 & BV421 & BioLegend & MF-14 \\
\hline IFNy & PE & BioLegend & XMG1.2 \\
\hline Granzyme B & FITC & BioLegend & GB11 \\
\hline Ly6C & BV780 & BioLegend & HK1.4 \\
\hline MHC-II & PerCP/Cy5.5 & BioLegend & AF6-120.1 \\
\hline NRP1 & BV421 & BioLegend & 3E12 \\
\hline NRP1 & PE & BioLegend & 3E12 \\
\hline $\begin{array}{l}\mathrm{H}-2 \mathrm{D}^{\mathrm{B}}-\mathrm{NP} \\
\text { tetramer }\end{array}$ & PE-Cy7 & Made in-house & \\
\hline PlexinA1 & PE & R\&D Systems & FAB4309P \\
\hline PlexinA2 & APC & R\&D Systems & FAB5486A \\
\hline PlexinA4 & $\begin{array}{l}\text { Conjugated to } \\
\text { PE }\end{array}$ & Abcam & ab39350 \\
\hline Podoplanin & APC & BioLegend & 8.1 .1 \\
\hline TCRb & APC/Cy7 & BioLegend & H57-597 \\
\hline TCRb & PE-CF594 & $\mathrm{BD}$ & H57-597 \\
\hline TGFbRI & PE & R\&D Systems & FAB5871P \\
\hline TGFbRII & PE & R\&D Systems & FAB532P \\
\hline TNFa & \begin{tabular}{|l|} 
PerCP/Cy5.5 \\
\end{tabular} & BioLegend & MP6-XT22 \\
\hline Sema3A & FITC, PE & R\&D Systems & IC1250P \\
\hline VEGFR2 & PE & BioLegend & Avas12 \\
\hline Zombie & Violet Dye & BV421 & BioLegend \\
\hline Zombie & Aqua Dye & BV525 & BioLegend \\
\hline Zombie & Near-infrared & APC-780 & BioLegend \\
\hline
\end{tabular}


600

RNA was extracted from cells using RNeasy kit (QIAGEN), followed by quantification on Nanodrop (Thermo Scientific). RNA was reverse transcribed using QuantiTect Reverse Transcription Kit (QIAGEN). Both controls without RNA or reverse transcription were included, and all experiments were performed in minimum technical triplicates and biological duplicates. cDNA was diluted to $10-20 \mathrm{ng}$ in $5 \mathrm{ul} /$ well and added to qRT-PCR plates. Taqman probes were combined with 2x Fast Taqman Master Mix and $5 \mathrm{ul} /$ well added to the cDNA. qRT-PCRs were run on a QuantStudio7 qRT-PCR machine (Life Technologies). Expression was normalized to the house keeping gene HPRT.

The following TagMan probes were used: BNIP3 (Mm01275600-g1), HPRT (Mm03024075m1), PDK1 (Mm00554300-m1), PDL1 (Mm00452054-m1), SEMA3A (Mm00436469-m1) and VEGFA (Mm00437306-m1).

\section{Western blot}

Cells were washed in PBS and pelleted, before being resuspended in lysis buffer with a EDTA protease inhibitor for at least 30 minutes on ice to extract protein. Cell-debris was removed by centrifugation at $4^{\circ} \mathrm{C}$. Supernatant containing protein was collected and quantified using Pierce BCA protein assay using diluted albumin as a standard.

Samples were mixed with Loading Buffer (Life Technologies) and Reducing Agent (Life Technologies) and heated to $95^{\circ} \mathrm{C}$ for at least 5 minutes. $4-12 \%$ Bis-Tris gels and MES SDS Buffer (Life Technologies) were used for proteins with a molecular weight below $200 \mathrm{kDa}$, while proteins above $200 \mathrm{kDa}$ were blotted on a 3-8\% Tris-Acetate gels in MOPS Buffer (Life Technologies). Proteins were separated at 200V for approximately one hour and transferred onto either PVDF or nitrocellulose membrane using the TransBlot Turbo Transfer (BioRad) system. Gels were blocked in 5\% BSA/PBS solution (blocking buffer) for at least 30 minutes at RT. Membranes were stained with primary antibody in fresh blocking buffer and incubated at $4 \mathrm{C}$ overnight on a shaker, washed five times in PBS with Triton-X $(0.1 \%$ Tween-20 in PBS) followed by incubation with fluorescent secondary antibodies (LICOR) diluted 1:20000 in blocking buffer for 1 hour on a shaker. Membranes were dried and imaged using the Odyssey Near-Infrared imaging system (LI-COR). 
The following antibodies were used: Anti-Neuropilin 1 antibody (Abcam, ab184783), antiPlexinA1 (R\&D Systems, AF4309), anti-PlexinA2 (R\&D Systems, AF5486), anti-GAPDH (Santa Cruz, sc-32233), anti- $\beta$-Actin (Cell Signaling Technology, 13E5).

632

\section{CRISPR/Cas9 editing and verification of B16.F10.Ova cells}

A sgRNA targeting Sema3a was cloned into Cas9-2A-EGFP expression vector $\mathrm{pX} 458$. This vector was electroporated into 1x10 B16.F10.Ova cells suspended in Solution V (Lonza) using the Amaxa 2B nucleofector (Lonza) with settings P-020. After 48 hours, single cells were sorted using the SH800 cell sorter (SONY) and expanded. Clones were genotyped by high-throughout sequencing. Briefly, the targeted locus was PCR amplified from each clone and subsequently indexed with a unique combination of i5 and i7 adaptor sequences. Indexed amplicons were sequenced on the MiSeqV2 (Illumina) and demultiplexed reads from each clone were compared to the wildtype Sema3a reference sequence using the CRISPResso webtool (62).

\section{Lentiviral transduction of cells}

A lentiviral Sema3A overexpression vector was purchased from VectorBuilder. To generate viral particles, this transfer vector was co-tranfected into HEK-293T cells along with the packaging and envelope plasmids pCMV-dR8.91 and pMD2.G using polyethylenimine. Crude viral supernatant was filtered and used to transduced B16.F10.Ova cells. mCherry positive transduced cells were selected by FACS using the SH800 cell sorter (SONY) to create a Sema3A overexpression cell line.

\section{Protein production of Sema3A and mutant Sema3A}

Recombinant mouse Sema3As-P (residues 21-568), Sema3As-P-I (residues 21-675, without a HIS tag) along with the Nrp1-binding deficient mutant Sema3A (residues 21-568, L353NP355S), here called mutant Sema3A, were cloned into a pHLsec vector optimized for large scale protein production as described before (63). The L353N P355S mutation in mutant Sema3A introduces an N-linked glycan to the Sema3A-Nrp1 interaction site, which is sufficient to block the formation of Sema3A-Nrp1-PIxnA2 signaling complex as described previously (5). Additionally, in all proteins, furin sites (R551A and R555A) were removed to 
prevent Sema3A proteolytic processing and enable more controlled purification and sample homogeneity. Proteins were expressed in HEK293T cells. Proteins were purified from bufferexchanged medium by immobilized metal-affinity followed by size-exclusion chromatography using a Superdex 200 column (GE).

For some experiments, purified Sema3A protein was labelled with AF647 using Alexa Fluor 647 Antibody Labeling Kit according to protocol (Invitrogen, cat. no A20186) at a F/P rate at $1-2$.

\section{Live-cell imaging of cells for migration studies, LifeAct and IRM quantification}

u-Slide I 0.4 Luer (Cat. no. 80172, Ibidi), u-Slide 8 well (Cat. no. 80826, Ibidi) and u-Slide Angiogenesis (Cat. no. 81506, Ibidi) were used for live-cell imaging of T cell motility and adhesion.

Proteins were immobilized on the plates by resuspension in PBS and allowing them to adhere for 2 hours as room-temperature, followed by three washes in PBS with 1-2\% BSA. The following proteins and concentrations were used: ICAM-1-Fc (Cat. 553006, BioLegend, at $10 \mu \mathrm{g} / \mathrm{mL}$ ), CD3 (clone 145-2C11, BioLegend, at 10ug/mL), CXCL12 (Cat. 578702, BioLegend, at $0.4 \mu \mathrm{g} / \mathrm{mL}$, BioLegend). Sema3As-p and mutant Sema3A (described above) were either ligated to surfaces in a similar manner or added to the medium while imaging at $5 \mu \mathrm{M}$. Plates were used immediately after preparation.

For experiments, $\mathrm{T}$ cells were activated for 48 hours using peptide stimulation, sorted and washed. In cases were aNRP1 or isotype control treatment was applied, T cells were incubated with these antibodies for 15 minutes at $37 \circ \mathrm{C}$, before an additional wash. T cell medium without phenol red and IL-2 was prewarmed and used to resuspend cells. Cells were added to plates placed on a stage in environmental chamber set at 37C directly at the microscope. DeltaVision Elite Live cell imaging microscope, Zeiss LSM 780 or 880 confocal microscopes were used with a Zeiss Plan-Neofluar 10x (0.3 NA), 40x (0.6 NA)or 63x (1.3 NA) lens.

In cases where T cells were tested under shear stress, cells were loaded into Hamilton syringes, and installed on a Harvard Apparatus PHD 2000 pump, and connected to chambered slides through 0.4 luer tubes. Flow rates and control of directionality of flow (ie, 
690

691

692

693

694

695

696

697

698

699

700

701

702

703

704

705

706

707

708

709

710

711

712

713

714

715

716

717

718

719

720

that it was laminar) were measured using fluorescent beads over set distances to calibrate instrument.

In cases were $T$ cells were treated with drugs while imaging, drugs were first resuspended in T cell medium and carefully added on top of solution while images were being acquired.

Care was taken always to take into consideration the correct concentration under growing levels of media. The following drugs were used: Jasplakinolide (Cat. J4580, Sigma, used at 5uM) and Blebbistatin (Cat. 203390, EMD Millipore, used at $100 \mathrm{uM}$ ), as well as Sema3As$\mathrm{P}$ (used at 1-5 $\mu \mathrm{M}$ ).

Data was acquired at $1 \mathrm{sec}$ to 1 minute per frame as indicated, and analyzed in Fij//mageJ (64). For cell tracking and visualization of spiderplots the Trackmate package was used (65). Subsequent tracks were analyzed in $\mathrm{R}(60)$ and visualized using the ggplot2 package (66). For cell contours, IRM and LifeAct, data was thresholded and collected in Fij/ImageJ, then exported to $\mathrm{R}$ for analysis and visualization. For quantification of IRM and LifeAct area while adding drugs, movies were edited such that the analyzed frames were equal to the timing indicated in figures (ie to start 3-5 minutes before adding the first drug). In videos with a frame-rate of 1 frame per second, 3 frames around the frame in which drugs was added was removed to avoid blurry or distorted images.

\section{Transwell chemotaxis assay}

Trans-migration was assessed in 24-well transwell plates with 3- $\mu \mathrm{m}$ pore size (Corning Life Sciences). The lower chamber was loaded with $500 \mu \mathrm{l}$ T cell medium with $10 \mathrm{IU}$ IL-2 and with or without $50 \mathrm{ng} / \mathrm{mL}$ CXCL10. $10^{5} \mathrm{CD} 8+$ OT-I CD8+ cells were stimulated ans sorted as described above and added in a volume of $100 \mu \mathrm{l}$ of T cell medium to the upper chamber, in either the presence of a blocking aNRP1 antibody or an isotype control, and $5 \mu \mathrm{M}$ Sema3As-p. As a positive control, effector cells were placed directly into the lower chamber. As a negative control, migration medium alone was placed in the upper chamber. Plates were incubated for 3 hours at $37 \mathrm{C}$ in a $5 \% \mathrm{CO}_{2}$ atmosphere. Thereafter, the Transwell inserts were removed and the contents of the lower compartment were recovered. Cells from the lower chamber were stained and the cells were quantified by flow cytometry.

\section{Immunological synapses analysis on supported lipid bilayers (SLB)}


721 The concentrations of the ligands used were as follows: $5 \mu \mathrm{g} / \mathrm{ml}$ to achieve 10

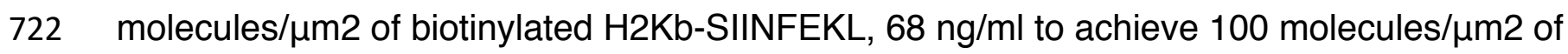
723 12x-HIS-tagged CD80 (AF488-labelled), and $122 \mathrm{ng} / \mathrm{ml}$ to achieve $200 \mathrm{molecules} / \mu \mathrm{m} 2$ of 724 12x-His tagged ICAM-1 (AF405-labelled). These concentration were determined based on 725 titrations on bead supported bilayers analyzed by flow cytometry. Sema3As-P-ı (described 726 above) was used, as this protein had no HIS-tag and so could not interfere with binding of 727 other tagged proteins in bilayer.

728 For live cell imaging, supported lipid bilayer presenting H2Kb-SIINFEKL, CD80, and ICAM7291 were assembled in sticky-Slide VI 0.4 luer (Ibidi) channels. The entire channel was filled 730 with a liposome suspension to form bilayer all along the channel. Live cells on SLBs were 731 imaged using the Olympus FluoView FV1200 confocal microscope that was enclosed in an 732 environment chamber at $37 \circ \mathrm{C}$ and operated under standard settings. $60 \mathrm{x}$ oil immersion 733 objective (1.4 NA) was used with $2 x$ digital zoom for time-lapse imaging at 20 second 734 intervals.

735 For fixed cell imaging, SLBs presenting H2Kb-SIINFEKL, CD80, and ICAM-1 were 736 assembled in 96-well glass-bottom plates (MGB096-1-2-LG-L, Brooks). 50,000 cells were 737 introduced into the wells at 37C and fixed 10 minutes later by adding 8\% PFA in 2x PHEM 738 buffer. After 3 washes with $0.1 \%$ BSA in HBS the fixed cells were imaged on the InCell 6000 739 wide-field fluorescence high-throughput imaging station using a 40x air objective (0.75 NA). 740 The imaging station was programmed to visit specific equivalent locations in each of the 741 desired wells in the 96-well plate.

742 Analysis of fixed cell images was carried out by the MATALB based TIAM HT package (26). 743 The source-code is available on the github repository: https://github.com/uvmayya/TIAM HT.

745 Acquisition of tissue from ccRCC patients

746 Acquisition and analysis of ccRCC samples were approved by Oxfordshire Research Ethics 747 Committee C. After informed written consent was obtained, samples were collected, and 748 store until use by Oxford Radcliffe Biobank (project reference 17/A100 and 16/A075). 
751 cDNA from single cells was obtained using a modified version of the SmartSeq2 protocol 752 (67). Briefly, single cells were sorted into plates containing lysis buffer. cDNA was generated 753 by template switch reverse transcription using SMARTScribe Reverve Transcriptase 754 (Clontech), a template-switch oligo and primers designed for the constant regions of Trac 755 and Trbc genes. TCR amplification was achieved by performing two rounds of nested PCR 756 using Phusion High-Fidelity PCR Master Mix (New England Biolabs). During the first PCR 757 priming, indexes were included, to identify each cell. A final PCR was performed to add the 758 Illumina adaptors. TCR libraries were sequenced on Illumina MiSeq using MiSeq Reagent 759 Kit V2 300-cycle (Illumina). FASTQ files were demultiplexed for each cell. Sequences from 760 clones were analysed using MiXCR (68). Post analysis was performed using VDJtools (69).

\section{CT-tetramer staining}

HLA-A2 monomers were made in-house, and loaded with CT-antigens through UV-directed ligand exchange using published protocols (70). Following ligand exchange, all monomers were tetramerized through binding to PE-Streptavidin, washed, and combined to allow for "cocktail" staining of cells. Frozen vials of tumor tissue were thawed, dissociated, and CD45positive sorted, followed by staining with $0.5 \mu \mathrm{g}$ of each tetramer in $50 \mathrm{ul}$ for one hour at RT. Otherwise staining proceeded like described previously.

The following CT-antigens were loaded into HLA-A2 monomers:

\begin{tabular}{|l|l|}
\hline Ligand & Antigen \\
\hline MLMAQEALAFL & LAGE-1 \\
\hline SLLMWITQC & LAGE-1 \\
\hline SLLMWITQA & NY-ESO-1 \\
\hline KVLEYVIKV & MAGE-A1 \\
\hline GLYDGMEHL & MAGE-A10 \\
\hline YLQLVFGIEV & MAGE-A2 \\
\hline FLWGPRALV & MAGE-A3 \\
\hline KVAELVHFL & MAGE-A3 \\
\hline FLWGPRALV & MAGE-A3 \\
\hline
\end{tabular}




\begin{tabular}{|l|l|}
\hline ALKDVEERV & MAGE-C2 \\
\hline KVLEFLAKL & MAGE-C2 \\
\hline LLFGLALIEV & MAGE-C2 \\
\hline ELAGIGILTV & MART1 \\
\hline TLNDECWPA & Meloe-1 \\
\hline AMAPIKVRL & PRDX5 (OMT3-12) \\
\hline LKLSGVVRL & RAGE-1 \\
\hline KASEKIFYV & SSX2 \\
\hline SVYDFFVWL & TRP2 \\
\hline YMDGTMSQV & Tyrosinase \\
\hline CQWGRLWQL & BING4 \\
\hline VLPDVFIRCV & GnT-V \\
\hline
\end{tabular}

Immunohistochemistry and image acquisition and analysis

773 The diagnostic hematoxylin and eosin (H\&E) stained slides for 12 cases of clear cell renal cell carcinoma were reviewed to identify corresponding formalin-fixed paraffin embedded tissue blocks that contained both tumor and adjacent non-tumor tissue. Strictly serial $4 \mu \mathrm{m}$ sections were then cut from the most appropriate block from each case. These sections underwent immunohistochemistry staining on a Leica BOND-MAX automated staining machine (Leica Biosystems). Briefly, sections were deparaffinized, underwent epitope retrieval and endogenous peroxidase activity was blocked with $3 \%$ hydrogen peroxide (5 minutes). Subsequently, sections were incubated with the primary antibody (30 minutes) followed by post-primary and polymer reagents (8 minutes each). Next, 3,3'Diaminobenzidine (DAB) chromogen was applied (10 minutes) (all reagents contained within the BOND Polymer Refine Detection kit, Leica Biosystems, catalogue no. DS9800). For double immunohistochemistry staining, the above cycle was repeated twice with the first cycle using Fast red chromogen labelling (all reagents contained within the BOND Polymer Refine Red Detection kit, Leica Biosystems, catalogue no. DS9390) and the second cycle $\mathrm{DAB}$ chromogen labelling. At the end of both the single and double immunohistochemistry protocols, the sections were counterstained with hematoxylin (5 minutes), mounted with a glass coverslip and left to dry overnight. The following primary antibodies were used during 
staining: CD31 (Agilent Technologies, JC70A, 1:800), CD8 (Agilent Technologies, C8/144B, 1:100) and Sema3A (Abcam, EPR19367, cat. no. ab199475, 1:4000).

Stained slides were scanned at $\times 400$ magnification using the NanoZoomer $\$ 210$ digital slide scanner (Hamamatsu). Sema3A-stained digital images were reviewed by a trained pathologist (PSM) and the extent of staining was quantified in the regions where expression was deemed to be highest ('Sema3A-rich') and lowest (Sema3A-poor) within the same tumor, using custom-made Matlab (MathWorks) scripts $(\%$ staining $=\mathrm{DAB}+$ pixels/total pixels x 100; raw data, image analysis, and data processing scripts are available upon request). This analysis was repeated in the same regions on adjacent serial sections for CD31 (as for Sema3A) and CD8 (for which discrete cell counts were calculated from stained regions using a water shedding process).

\section{Statistical analysis}

Statistical analysis was performed in Prism software (GraphPad) or R (60). Data was tested for Gaussian distribution. For multiple comparisons, either one-way or two-way analysis of variance (ANOVA) was used with Tukey's test to correct for multiple comparisons. For comparison between two groups, Students t test, Student's paired t test, or one-tailed or two-tailed Mann-Whitney test were used.

\section{AUTHORS CONTRIBUTIONS}

Conceptualization: M.B.B, V.C., E.Y.J., M.L.D., T.A.F., M.F.; Formal analysis: M.B.B., Y.S.M., V.A., M.R., P.S.M., V.M., M.F.; Funding acquisition: V.C., E.Y.J., M.L.D., T.A.F., M.F., C.W.P.; Investigation: M.B.B., Y.S.M., V.A., P.S.M., U.G., S.V., M.R., C.K., J.C., A.V.H., V.M., P.R., L.R.O., M.F., H.C.Y.; Methodology: V.C., U.G., M.B.B., M.L.D., M.F., H.C.Y., Y.S.M., A.R.T.; Project administration: V.C.; Resources: U.G., S.V., V.J., M.R., V.W., Y.K., V.M., A.R.T.; Software: M.R., J.A.B., M.B.B.; Supervision: V.C.; Visualization: M.B.B., P.S.M., V.M., M.F.; Writing - original draft: M.B.B., V.C., E.Y.J., M.L.D., M.F.; Writing review \& editing: M.B.B., V.C., E.Y.J., M.L.D., M.F., Y.S.M., C.K., P.S.M., V.J. 


\section{ACKNOWLEDGEMENTS}

The authors wish to thank members of the Vincenzo Cerundolo and Tudor A. Fulga laboratories, University of Oxford, for helpful discussions and suggestions. Simon Davis. Oliver Bannard and Audrey Gérard, University of Oxford, for helpful advice and guidance. The staff of the University of Oxford Department of Biomedical Services for animal husbandry. Christoffer Lagerholm and Dominic Waithe of the Wolfson Imaging Centre at University of Oxford for microscopy training and support. The Medical Research Council (MRC) WIMM Flow Cytometry Facility for training and support. The Oxford Centre for Histopathology Research and the Oxford Radcliffe Biobank, which are supported by the NIHR Oxford Biomedical Research Centre and the Kennedy Institute of Rheumatology Microscopy Facility. Lisa Browning, Oxford University Hospital, for examination of histology samples. David Pinto, University of Oxford, for code for analysis of CDR3 sequences.

This work was supported by the U.K. MRC (MRC Human Immunology Unit), the Oxford Biomedical Research Centre, and Cancer Research UK (Programme Grant C399/A2291 to V.C.; C375/A17721 to E.Y.J.); the Wellcome Trust (212343/Z/18/Z for M.F.; 100262Z/12/Z for M.L.D. and S.V.) and Kennedy Trust for Rheumatology Research (to M.L.D. and S.V.), European Commission (ERC-2014-AdG_670930 for M.L.D. and V.M.), Cancer Research Institute (to V.M.), and EPSRC (EP/S004459/1 for M.F. and H.C.Y). The Wellcome Centre for Human Genetics is supported by Wellcome Trust Centre grant 203141/Z/16/Z. P.S.M. is supported by a Jean Shanks Foundation/Pathological Society of Great Britain \& Ireland Clinical Research Training Fellowship. C.K. is supported by a Wellcome Studentship (105401/Z/14/Z). V.J. is supported by an EMBO Long-Term Fellowship (ALTF 1061-2017).

J.A.B. is supported by EPSRC/MRC Centre for Doctoral Training in Systems Approaches to Biomedical Science (EP/G037280/1) and the EPSRC Impact Acceleration Account (EP/R511742/1). L.R.O. is supported by the Independent Research Fund Denmark (804800078B).

\section{COMPETING INTERESTS}

Authors declare no competing interests. 


\section{REFERENCES AND NOTES}

8501

J. A. Joyce, D. T. Fearon, T cell exclusion, immune privilege, and the tumor microenvironment. Science. 348, 74-80 (2015).

G. Serini, D. Valdembri, S. Zanivan, G. Morterra, C. Burkhardt, F. Caccavari, L. Zammataro, L. Primo, L. Tamagnone, M. Logan, M. Tessier-Lavigne, M. Taniguchi, A. W. Puschel, F. Bussolino, Class 3 semaphorins control vascular morphogenesis by inhibiting integrin function. Nature. 424, 391-397 (2003). repulsion and invasion. J Clin Invest. 109, 993-998 (2002). semaphorin-3A receptors. Cell. 99, 59-69 (1999). Jones, Neuropilins lock secreted semaphorins onto plexins in a ternary signaling complex. Nat Struct Mol Biol. 19, 1293-1299 (2012). colocalization during growth cone collapse. J Cell Biol. 149, 411-422 (2000). react to semaphorin $3 \mathrm{~A}$ application through a biphasic response that requires multiple myosin II isoforms. Mol Biol Cell. 20, 1167-1179 (2009). Semaphorin-3A is expressed by tumor cells and alters T-cell signal transduction and function. Blood. 107, 3321-3329 (2006).

874 The EGR2 targets LAG-3 and 4-1BB describe and regulate dysfunctional antigen-specific 875 CD8+ T cells in the tumor microenvironment. J Exp Med. 214, 381-400 (2017). 
Schietinger, Chromatin states define tumour-specific T cell dysfunction and reprogramming. Nature. 545, 452-456 (2017).

11.

M. Leclerc, E. Voilin, G. Gros, S. Corgnac, V. de Montpreville, P. Validire, G. Bismuth, F. Mami-Chouaib, Regulation of antitumour CD8 T-cell immunity and checkpoint blockade immunotherapy by Neuropilin-1. Nat Commun. 10, 3345 (2019).

12.

Y. Lepelletier, I.

C. Moura, R. Hadj-Slimane, A.

A. Renand, S. Fiorentino, C.

Baude, A. Shirvan, A. Barzilai, O. Hermine, Immunosuppressive role of semaphorin-3A on T cell proliferation is mediated by inhibition of actin cytoskeleton reorganization. Eur $J$ Immunol. 36, 1782-1793 (2006).

13.

M. Wallerius, T. Wallmann, M. Bartish, J. Ostling, A. Mezheyeuski, N. P. Tobin, E. Nygren, P. Pangigadde, P. Pellegrini, M. L. Squadrito, F. Ponten, J. Hartman, J. Bergh, A. De Milito, M. De Palma, A. Ostman, J. Andersson, C. Rolny, Guidance Molecule SEMA3A Restricts Tumor Growth by Differentially Regulating the Proliferation of TumorAssociated Macrophages. Cancer Res. 76, 3166-3178 (2016). metastatic dissemination induced by antiangiogenic treatment in mice. J Clin Invest. 122, 1832-1848 (2012).

15. N. Gioelli, F. Maione, C. Camillo, M. Ghitti, D. Valdembri, N. Morello, M.

Darche, L. Zentilin, G. Cagnoni, Y. Qiu, M. Giacca, M. Giustetto, M. Paques, I. Cascone, G. Musco, L. Tamagnone, E. Giraudo, G. Serini, A rationally designed NRP1-independent superagonist SEMA3A mutant is an effective anticancer agent. Sci Trans/ Med. 10 (2018), doi:10.1126/scitransImed.aah4807. 16. A. W. Goldrath, Immunological Genome Project Consortium, Transcriptional insights into the CD8(+) T cell response to infection and memory T cell formation. Nat. Immunol. 14, 404-412 (2013).

17. D. Zehn, S. Y. Lee, M. J. Bevan, Complete but curtailed T-cell response to very low-affinity antigen. Nature. 458, 211-214 (2009).

18.

Y. Glinka, G. J. Prud'homme, Neuropilin-1 is a receptor for transforming growth factor beta-1, activates its latent form, and promotes regulatory T cell activity. $J$ 
Leukoc Biol. 84, 302-310 (2008).

19.

M. V. Gelfand, N. Hagan, A. Tata, W.-J. Oh, B. Lacoste, K.-T. Kang, J. Kopycinska, J. Bischoff, J.-H. Wang, C. Gu, Neuropilin-1 functions as a VEGFR2 coreceptor to guide developmental angiogenesis independent of ligand binding. Elife. 3, e03720 (2014).

20. L. Tamagnone, S. Artigiani, H. Chen, Z. He, G. I. Ming, H. Song, A. Chedotal, M. L. Winberg, C. S. Goodman, M. Poo, M. Tessier-Lavigne, P. M. Comoglio, Plexins Are a Large Family of Receptors for Transmembrane, Secreted, and GPI-Anchored Semaphorins in Vertebrates. Cell. 99, 71-80 (1999).

21. Y. Lepelletier, S. Smaniotto, R. Hadj-Slimane, D. M. S. Villa-Verde, A. C. Nogueira, M. Dardenne, O. Hermine, W. Savino, Control of human thymocyte migration by Neuropilin-1/Semaphorin-3A-mediated interactions. Proc Natl Acad Sci U S A. 104, 55455550 (2007).

22. A. Casazza, D. Laoui, M. Wenes, S. Rizzolio, N. Bassani, M. Mambretti, S. Deschoemaeker, J. A. Van Ginderachter, L. Tamagnone, M. Mazzone, Impeding macrophage entry into hypoxic tumor areas by Sema3A/Nrp1 signaling blockade inhibits angiogenesis and restores antitumor immunity. Cancer Cell. 24, 695-709 (2013).

23. H. Takamatsu, N. Takegahara, Y. Nakagawa, M. Tomura, M. Taniguchi, R. H. Friedel, H. Rayburn, M. Tessier-Lavigne, Y. Yoshida, T. Okuno, M. Mizui, S. Kang, S. Nojima, T. Tsujimura, Y. Nakatsuji, I. Katayama, T. Toyofuku, H. Kikutani, A. Kumanogoh, Semaphorins guide the entry of dendritic cells into the lymphatics by activating myosin II. Nat Immunol. 11, 594-600 (2010).

24. M. Fritzsche, R. A. Fernandes, V. T. Chang, H. Colin-York, M. P. Clausen, J. H. Felce, S. Galiani, C. Erlenkamper, A. M. Santos, J. M. Heddleston, I. Pedroza-Pacheco, D. Waithe, J. B. de la Serna, B. C. Lagerholm, T.-L. Liu, T.-L. Chew, E. Betzig, S. J. Davis, C. Eggeling, Cytoskeletal actin dynamics shape a ramifying actin network underpinning immunological synapse formation. Sci Adv. 3, e1603032 (2017). 25. A. Orimo, P. B. Gupta, D. C. Sgroi, F. Arenzana-Seisdedos, T. Delaunay, R. Naeem, V. J. Carey, A. L. Richardson, R. A. Weinberg, Stromal fibroblasts present in invasive human breast carcinomas promote tumor growth and angiogenesis through elevated SDF-1/CXCL12 secretion. Cell. 121, 335-348 (2005). 
26. S. Valvo, V. Mayya, E. Seraia, J. Afrose, H. Novak-Kotzer, D. Ebner, M. L.

941 Dustin, Comprehensive Analysis of Immunological Synapse Phenotypes Using Supported 942 Lipid Bilayers. Methods Mol Biol. 1584, 423-441 (2017).

943 27.

Y. Ueda, N. Kondo, M. Ozawa, K. Yasuda, T. Tomiyama, T. Kinashi,

Sema3e/Plexin D1 Modulates Immunological Synapse and Migration of Thymocytes by Rap1 Inhibition. J Immunol. 196, 3019-3031 (2016).

94628.

W. A. Comrie, A. Babich, J. K. Burkhardt, F-actin flow drives affinity maturation and spatial organization of LFA-1 at the immunological synapse. J Cell Biol. 208, 475-491 (2015).

A. T. Ritter, S. M. Kapnick, S. Murugesan, P. L. Schwartzberg, G. M. Griffiths, J. Lippincott-Schwartz, Cortical actin recovery at the immunological synapse leads to termination of lytic granule secretion in cytotoxic T lymphocytes. Proc Natl Acad Sci U S A. 114, E6585-E6594 (2017).

95330.

A. F. Carisey, E. M. Mace, M. B. Saeed, D. M. Davis, J. S. Orange, Nanoscale Dynamism of Actin Enables Secretory Function in Cytolytic Cells. Curr Biol. 28, 489502.e9 (2018). dynamics. Nat Methods. 7, 168-169 (2010). activation and non-canonical catalysis of the Rap GTPase activating protein domain of plexin. Elife. 2, e01279 (2013). adhesion and cell motility through the regulation of myosin II. J Cell Biol. 176, 1021-1033 964 (2007). potentiates endothelial cell junctions by spatially controlling myosin II activity and actin 967 organization. J Cell Biol. 202, 901-916 (2013).

968 35. A. Mikulich, S. Kavaliauskiene, P. Juzenas, Blebbistatin, a myosin inhibitor, is 969 phototoxic to human cancer cells under exposure to blue light. Biochim Biophys Acta. 1820, 870-877 (2012). 
971 36. M. Ciofani, J. C. Zuniga-Pflucker, The thymus as an inductive site for T 972 lymphopoiesis. Annu Rev Cell Dev Biol. 23, 463-493 (2007).

973 37. T. J. Powell, J. D. Silk, J. Sharps, E. Fodor, A. R. M. Townsend, Pseudotyped 974 influenza A virus as a vaccine for the induction of heterotypic immunity. $J$ Virol. 86, 1339797513406 (2012).

976 38. M. A. Curran, W. Montalvo, H. Yagita, J. P. Allison, PD-1 and CTLA-4 977 combination blockade expands infiltrating $\mathrm{T}$ cells and reduces regulatory $\mathrm{T}$ and myeloid 978 cells within B16 melanoma tumors. Proc Natl Acad Sci U S A. 107, 4275-4280 (2010).

979 39. $\quad$ S. Chen, L.-F. Lee, T. S. Fisher, B. Jessen, M. Elliott, W. Evering, K. Logronio, 980 G. H. Tu, K. Tsaparikos, X. Li, H. Wang, C. Ying, M. Xiong, T. VanArsdale, J. C. Lin, 981 Combination of 4-1BB agonist and PD-1 antagonist promotes antitumor effector/memory 982 CD8 T cells in a poorly immunogenic tumor model. Cancer Immunol Res. 3, 149-160 983 (2015).

984 40. M. Z. Noman, B. Janji, B. Kaminska, K. Van Moer, S. Pierson, P. Przanowski, 985 S. Buart, G. Berchem, P. Romero, F. Mami-Chouaib, S. Chouaib, Blocking hypoxia986 induced autophagy in tumors restores cytotoxic T-cell activity and promotes regression. 987 Cancer Res. 71, 5976-5986 (2011).

988 41. $\quad$ B. Youngblood, K. J. Oestreich, S.-J. Ha, J. Duraiswamy, R. S. Akondy, E. E. 989 West, Z. Wei, P. Lu, J. W. Austin, J. L. Riley, J. M. Boss, R. Ahmed, Chronic virus infection 990 enforces demethylation of the locus that encodes PD-1 in antigen-specific CD8(+) T cells. 991 Immunity. 35, 400-412 (2011).

$99242 . \quad$ P. Jantzer, D. J. Schendel, Human renal cell carcinoma antigen-specific CTLs: 993 antigen-driven selection and long-term persistence in vivo. Cancer Res. 58, 3078-3086 994 (1998).

99543.

B. Yin, Y. Zeng, X. Wang, G. Liu, M. Zhang, Y. Song, Expression and clinical 996 significance of cancer-testis genes in clear cell renal cell carcinoma. Int J Clin Exp Pathol. 7, 4112-4119 (2014).

99844.

P. G. Coulie, B. J. Van den Eynde, P. van der Bruggen, T. Boon, Tumour 999 antigens recognized by $\mathrm{T}$ lymphocytes: at the core of cancer immunotherapy. Nat Rev 1000 Cancer. 14, 135-146 (2014).

$100145 . \quad$ J. S. Allingham, R. Smith, I. Rayment, The structural basis of blebbistatin 
1002

1003

1004

1005

1006

1007

1008

1009

1010

1011

1012

1013

1014

1015

1016

1017

1018

1019

1020

1021

1022

1023

1024

1025

1026

1027

1028

1029

1030

1031

1032

inhibition and specificity for myosin II. Nat. Struct. Mol. Biol. 12, 378-379 (2005).

46.

J. Yi, X. S. Wu, T. Crites, J. A. Hammer, Actin retrograde flow and actomyosin II arc contraction drive receptor cluster dynamics at the immunological synapse in Jurkat $T$ cells. Mol. Biol. Cell. 23, 834-852 (2012).

47.

J. Hong, S. Murugesan, E. Betzig, J. A. Hammer, Contractile actomyosin arcs promote the activation of primary mouse T cells in a ligand-dependent manner. PLoS ONE. 12, e0183174 (2017).

48.

M. Murrell, P. W. Oakes, M. Lenz, M. L. Gardel, Forcing cells into shape: the mechanics of actomyosin contractility. Nat. Rev. Mol. Cell Biol. 16, 486-498 (2015). 49.

G. Gallo, RhoA-kinase coordinates F-actin organization and myosin II activity during semaphorin-3A-induced axon retraction. J. Cell. Sci. 119, 3413-3423 (2006).

50.

J. M. Weiss, A. M. Bilate, M. Gobert, Y. Ding, M. A. Curotto de Lafaille, C. N.

Parkhurst, H. Xiong, J. Dolpady, A. B. Frey, M. G. Ruocco, Y. Yang, S. Floess, J. Huehn, S. Oh, M. O. Li, R. E. Niec, A. Y. Rudensky, M. L. Dustin, D. R. Littman, J. J. Lafaille, Neuropilin 1 is expressed on thymus-derived natural regulatory T cells, but not mucosagenerated induced Foxp3+ T reg cells. J. Exp. Med. 209, 1723-1742, S1 (2012).

51.

G. M. Delgoffe, S.-R. Woo, M. E. Turnis, D. M. Gravano, C. Guy, A. E.

Overacre, M. L. Bettini, P. Vogel, D. Finkelstein, J. Bonnevier, C. J. Workman, D. A. A.

Vignali, Stability and function of regulatory $T$ cells is maintained by a neuropilin-1semaphorin-4a axis. Nature. 501, 252-256 (2013).

52. S. R. Jackson, M. Berrien-Elliott, J. Yuan, E. C. Hsueh, R. M. Teague, Neuropilin-1 expression is induced on tolerant self-reactive CD8+ T cells but is dispensable for the tolerant phenotype. PLoS ONE. 9, e110707 (2014).

53. W. Hansen, M. Hutzler, S. Abel, C. Alter, C. Stockmann, S. Kliche, J. Albert, T. Sparwasser, S. Sakaguchi, A. M. Westendorf, D. Schadendorf, J. Buer, I. Helfrich, Neuropilin 1 deficiency on CD4+Foxp3+ regulatory T cells impairs mouse melanoma growth. J. Exp. Med. 209, 2001-2016 (2012).

54. A. E. Overacre-Delgoffe, M. Chikina, R. E. Dadey, H. Yano, E. A. Brunazzi, G. Shayan, W. Horne, J. M. Moskovitz, J. K. Kolls, C. Sander, Y. Shuai, D. P. Normolle, J. M. Kirkwood, R. L. Ferris, G. M. Delgoffe, T. C. Bruno, C. J. Workman, D. A. A. Vignali, Interferon-ץ Drives Treg Fragility to Promote Anti-tumor Immunity. Cell. 169, 1130- 
1033

1034

1035

1036

1037

1038

1039

1040

1041

1042

1043

1044

1045

1046

1047

1048

1049

1050

1051

1052

1053

1054

1055

1056

105761.

1058

1059

1060

1061

1062

1063

60.

62.

63.

1141.e11 (2017).

55. L. Fahlén, S. Read, L. Gorelik, S. D. Hurst, R. L. Coffman, R. A. Flavell, F. Powrie, T cells that cannot respond to TGF-beta escape control by CD4(+)CD25(+) regulatory T cells. J. Exp. Med. 201, 737-746 (2005).

56. P. C. Tumeh, C. L. Harview, J. H. Yearley, I. P. Shintaku, E. J. M. Taylor, L. Robert, B. Chmielowski, M. Spasic, G. Henry, V. Ciobanu, A. N. West, M. Carmona, C. Kivork, E. Seja, G. Cherry, A. J. Gutierrez, T. R. Grogan, C. Mateus, G. Tomasic, J. A. Glaspy, R. O. Emerson, H. Robins, R. H. Pierce, D. A. Elashoff, C. Robert, A. Ribas, PD-1 blockade induces responses by inhibiting adaptive immune resistance. Nature. 515, 568571 (2014).

57. S. A. Rosenberg, R. M. Sherry, K. E. Morton, W. J. Scharfman, J. C. Yang, S. L. Topalian, R. E. Royal, U. Kammula, N. P. Restifo, M. S. Hughes, D. Schwartzentruber, D. M. Berman, S. L. Schwarz, L. T. Ngo, S. A. Mavroukakis, D. E. White, S. M. Steinberg, Tumor progression can occur despite the induction of very high levels of self/tumor antigen-specific CD8+ T cells in patients with melanoma. J Immunol. 175, 6169-6176 (2005).

58. L. Gautier, L. Cope, B. M. Bolstad, R. A. Irizarry, affy--analysis of Affymetrix GeneChip data at the probe level. Bioinformatics. 20, 307-315 (2004).

59. M. E. Ritchie, B. Phipson, D. Wu, Y. Hu, C. W. Law, W. Shi, G. K. Smyth, limma powers differential expression analyses for RNA-sequencing and microarray studies. Nucleic Acids Res. 43, e47 (2015).

R Development Core Team, R: A Language and Environment for Statistical Computing (R Foundation for Statistical Computing, Vienna, Austria, 2008; http://www.Rproject.org).

61. T. Li, J. Fan, B. Wang, N. Traugh, Q. Chen, J. S. Liu, B. Li, X. S. Liu, TIMER: A web server for comprehensive analysis of tumor-infiltrating immune cells. Cancer Res. 77, e108-e110 (2017).

K. Clement, H. Rees, M. C. Canver, J. M. Gehrke, R. Farouni, J. Y. Hsu, M. A. Cole, D. R. Liu, J. K. Joung, D. E. Bauer, L. Pinello, CRISPResso2 provides accurate and rapid genome editing sequence analysis. Nat. Biotechnol. 37, 224-226 (2019).

A. R. Aricescu, W. Lu, E. Y. Jones, A time- and cost-efficient system for high- 
1064 level protein production in mammalian cells. Acta Crystallogr. D Biol. Crystallogr. 62, 1065 1243-1250 (2006). biological-image analysis. Nat. Methods. 9, 676-682 (2012). extensible platform for single-particle tracking. Methods. 115, 80-90 (2017). New York, 2016; https://ggplot2.tidyverse.org).

Sandberg, Full-length RNA-seq from single cells using Smart-seq2. Nature Protocols. 9, 171-181 (2014). profiling. Nature Methods. 12, 380-381 (2015). Receptor Repertoires. PLOS Computational Biology. 11, e1004503 (2015). Immunol, in press, doi:10.1002/0471142735.im1816s87. 


\section{MAIN FIGURE LEGENDS}

1093

1094

1095

1096

1097

1098

1099

1100

1101

1102

1103

1104

1105

1106

1107

1108

1109

1110

1111

1112

1113

1114

1115

1116

1117

1118

1119

1120

1121

Figure 1. Tumor-specific CD8+ T cells up-regulate NRP1 and Plexin-A1 allowing for Sema3A binding.

A-B. Representative histogram of flow cytometric analysis of surface NRP1 expression on human NY-ESO-1-specific HLA-A2 restricted CD8+ T cells and murine OT-I CD8+ T cells following 48 hours stimulation with cognate peptides. Cells are gated on CD45, CD8 and TCR $\beta$. Experiment repeated three times.

C. Analysis of NRP1 up-regulation using peptides with varying TCR affinities. Cells are gated on CD45.1, CD8 and TCR 3 . Cells from 3 mice per group, experiment was performed once. Data indicate mean \pm SD.

D. Quantification of surface binding of Sema3As-p on naïve and 48 hour stimulated OT-I T cells. Cells are gated on CD8 and CD3. Experiment was repeated three times. Data indicate mean $\pm S D$ of representative experiment.

E. Confocal imaging of 48 hour stimulated OT-I T cells stained with AF647-labelled Sema3As-p shows that the protein can bind to the cell membrane (white arrow) and within the cell (black arrow).

F. Flow cytometric analysis of PD-1 and NRP1 expression on OT-I T cells 11 days after adoptive transfer in spleen, non-antigen expressing tumor (B16.F10) and antigenexpressing tumor (B16.F10.Ova) $(n=6)$. Data representative of two independent experiments and indicate mean \pm SD of six mice per group.

G. Schematic of NRP1 interactions partners (left). Flow cytometric analysis of expression of selected NRP1 interactions partners on OT-I T cells 11 days after adoptive transfer $(n=5)$ (right). Experiment was performed once. Data indicate mean \pm SD.

Abbreviations: gMFI, geometric mean fluorescence intensity. N4, SIINFEKL. Q4, SIIQFEKL. T4, SIITFEKL.

Figure 2. Sema3A negatively regulates CD8+ $T$ cell adhesion, motility and migration through NRP1. 
1122 A. Representative brightfield and IRM images of 48 hour stimulated OT-I T cells adhering to 1123 ICAM-1 and CXCL12 coated plates with either Sema3As-p or IgG immobilized.

1124 B. Quantification of contact area per single cell using live-cell microscopy for 10 minutes 1125 after OT-I T cells were added to plate ( $\mathrm{n}=60$ cells). Data representative of three independent 1126 experiments and indicate mean \pm SEM. ${ }^{* * *}=\mathrm{P}<0.0001$ by Student's t-test.

1127 C. Relative frequency of cell polarity from brightfield images. A polarity of 1 indicates a shape 1128 of a perfect circle, 0 a rectangular shape. Representative images of OT-I T cells illustrated 1129 above graph.

1130 D. Representative spider plots showing the migration paths of individual T cells pre-treated 1131 with either a NRP1 blocking antibody or isotype control antibody on similar plates as in (A).

1132 E. Graph of single cell distance (left) and single cell velocity (right) in same experiment as 1133 (D). ( $n=314-744$ cells per group). Data combined from five independent experiments indicate 1134 mean $\pm \mathrm{SD}$. ${ }^{* *}=\mathrm{P}<0.001$, ns $=$ not significant by Kruskal-Wallis test.

1135 F. Representative spider plots showing the migration path of individual OT-I T cells on similar 1136 plates as in $(A)$, with flow rates at 0 or $80 \mu \mathrm{m} / \mathrm{sec}$. Arrows indicate flow direction.

1137 G. Quantification of percent of OT-I cells that detach in same experiment as $(F)(n=20-73$ 1138 cells per condition). Data representative of two independent experiments and indicate mean $1139 \pm$ SEM. ${ }^{*}=\mathrm{P}<0.05,{ }^{* * *}=\mathrm{P}<0.001$, ns $=$ not significant by two-way ANOVA.

1140 H. Representative graph of number of stimulated OT-I T cells able to transmigrate through $11413 \mu \mathrm{m}$ Boyden chamber with CXCL12 in bottom chamber, with or without Sema3As-p in top1142 chamber. OT-I T cells were pre-treated with either a blocking NRP1 antibody or isotype 1143 control antibody. Data representative of two independent experiments and indicate mean \pm 1144 SD. ${ }^{* \star *}=\mathrm{P}<0.001$, ns = not significant, by two-way ANOVA.

Abbreviations: IRM, interference reflection microscopy. Sec, second.

Figure 3. Sema3A negatively regulates CD8+ T cells' immunological synapse formation.

1150 A. Live-cell imaging visualizing surface interface using IRM of stimulated CD8+ T cells 1151 dropped on an activating surface with immobilized ICAM-1 and CD3 and Sema3As-P or IgG 
1152 present in medium (left). Cell contour of representative cells from either condition (right). 1153 Color of contour indicates time from 0 to $200 \mathrm{sec}$ as denoted on scalebar.

1154 B. Quantification of maximum size of cell contact area (top) and spreading speed from initial 1155 contact to maximum contact area (bottom) ( $n=25$ cells per group) in same experiment as 1156 (A). Data combined from three independent experiments and indicate mean $\pm \mathrm{SD}$. ${ }^{* *}=\mathrm{P}<$ $11570.01,{ }^{* \star *}=\mathrm{P}<0.001$, by Mann-Whitney test.

1158 C. Live-cell imaging of activated T cells pre-treated with Sema3As-P-I-AF647 and allowed to 1159 form synapses on supported lipid bilayers with ICAM-1, CD80 and H-2Kb-SIINFEKL. Arrows 1160 in merged image indicate cells that have bound Sema3A and do not form immunological 1161 synapses.

1162 D. Representative image from high-throughput analysis of immunological synapses on 1163 supported lipid bilayers as in (C) with OT-I T cells pre-treated with Sema3A or not.

1164 E. Quantification of immunological synapses with or without Sema3As-P-ı pre-treated OT-I T 1165 cells. Data from six independent experiments ( $n=90-1100$ cells per mouse per group). ${ }^{* *}=$ $1166 \mathrm{P}<0.01$, by paired t-test.

Abbreviations: IRM, interference reflection microscopy. Sec, seconds.

Figure 4. Sema3A affects T cell actin dynamics through actomyosin II activity.

A. Representative flow cytometric analysis of F-actin content with no or varying exposure to Sema3As-p treatment in 48 hour stimulated OT-I T cells as measured by Phalloidin-staining.

1173 Percentage indicate positive cells in each condition. Data representative of two independent 1174 experiments.

1175 B. Representative brightfield images of 48 hour stimulated LifeAct OT-I T cells adhering to 1176 ICAM-1 and CD3 coated plates before and after Sema3As-p added to medium.

1177 C. Representative confocal images of LifeAct in OT-I T cells (left) and their contour plots 1178 (right) from same experiment as in (B). Image taken at cell-surface interface. Dashed white 1179 line indicate area used for (D). Color of contour indicates time from 0 to $300 \mathrm{sec}$ as denoted 1180 on scalebar. 
D. Kymograph before (top) and after (bottom) Sema3As-p added to medium on area indicated with white dashed line in (C). Dotted line along edge of cell denoted example of data used for calculating data in (E).

E. Quantification of F-actin velocity at cell edge before and after treatment with either Sema3As-p, Jasplakinolide or mutant Sema3A ( $n=25-33$ cells per group) using same experimental setup as in (B). Data combined from three independent experiments and indicate mean $\pm \mathrm{SD}$. ${ }^{\star \star \star *}=\mathrm{P}<0.0001$, ns $=$ not significant, by paired t-test.

F. Intensity plot of LifeAct signal before and after Sema3As-p treatment of a single OT-I T cell (left) or quantified on multiple cells exposed to Sema3As-p (right) using same experimental setup as in (B). Arrows indicate measured cell width. ${ }^{* *}=P<0.01$, by paired t-test.

G. Cell width dynamics measured like $(F)$ over time before (white background) or after (grey background) Sema3As-p, Jasplakinolide or mutant Sema3A addition to medium.

H. Quantification of IRM area of individual OT-I T cells (grey lines) or average for group (red line) over time, with no treatment (leftmost white background), under treatment with Sema3A (grey background) and then Blebbistatin (rightmost white background). Above representative contour plots of single cell under different treatments, with color denoting time (150 sec total). Cells were allowed to settle, and form contact for 3-5 min before data acquisition. Area normalized to cell area at $t=0 \mathrm{sec}$. Data combined from three independent experiments ( $\mathrm{n}=27$ cells). ${ }^{\star \star \star}=\mathrm{P}<0.001,{ }^{\star \star \star \star}=\mathrm{P}<0.0001$, ns $=$ not significant by two-way ANOVA at time-points 90,270 and $450 \mathrm{sec}$.

I. Quantificantion of IRM area of individual OT-I T cells and representative contour plots as in $(\mathrm{H})$, but with treatment with Blebbistatin (grey background) before Sema3As-P (rightmost white background). Data combined from three independent experiments ( $n=37$ cells). ${ }^{*}=P$ $<0.05$, ns $=$ not significant by two-way ANOVA at time-points 90,270 and $450 \mathrm{sec}$.

Abbreviations: Min, minutes. Sec, seconds. t, time.

Figure 5: Nrp1-deficiency enhances anti-tumor migration and activity of CD8+ $T$ cells.

A. Growth curve of B16.F10 cells in NRP1+/+, NRP1Flox/+ and NRP1Flox/Flox mice (left) and Kaplan-Meier survival curve (right). Dashed lines indicate growth in individual mice, bold line 
1212

1213

1214

1215

1216

1217

1218

1219

1220

1221

1222

1223

1224

1225

1226

1227

1228

1229

1230

1231

1232

1233

1234

1235

1236

1237

1238

1239

1240

1241

1242

average for group. Combined data from 4 independent experiments with 3-6 mice per group. ${ }^{* * *}=\mathrm{P}<0.001, \mathrm{~ns}=$ not significant by two-way ANOVA.

B. Growth curve of LL/2 cells in NRP1+/+, NRP1Flox/+ and NRP1Flox/Flox mice (left) and KaplanMeier survival curve (right) ( $n=3-6$ mice per group). Dashed lines indicate growth in individual mice, bold line average for group. Experiment performed once. ${ }^{\star \star *}=\mathrm{P}<0.001$, ns $=$ not significant by two-way ANOVA.

C. Growth curve of B16.F10 cells in NRP1Flox/+ and NRP1Flox/Flox mice pre-treated with either anti-CD8 antibody or isotype control ( $n=5-7$ mice per group). Dashed lines indicate growth in individual mice, bold line average for group. Data combined from two independent experiments. ${ }^{* * *}=\mathrm{P}<0.001$, ns $=$ not significant by two-way ANOVA.

D. Enumeration of CD4+ and CD8+ T cells infiltrated into B16.F10 tumors in NRP1+/, NRP1Flox/+ and NRP1Flox/Flox mice ( $n=6$ per group). Data indicate mean \pm SD. ${ }^{* *}=P<0.001$, ns $=$ not significant by two-way ANOVA.

E. Experimental setup of mixed bone-marrow chimeras in C57BL/6 mice (left) and subsequent enumeration of $C D 8+T$ cells in mice (middle graph). Ratio of CD8+ $T$ cells from NRP1Flox/Flox to NRP1Flox/+ bone-marrow derived cells (right graph) ( $n=6$ mice per group). Experiment performed once. Data indicate mean $\pm \mathrm{SD}$. ${ }^{*}=\mathrm{P}<0.05$ by one-way ANOVA.

F. Experimental setup using B16.F10 Sema3A KO or Sema3A OE cells (left) and growth curve of cells in untreated mice (right) ( $n=8$ mice). Experiment performed once. $n s=$ not significant by two-way ANOVA

G. Growth curve of B16.F10 Sema3A KO or Sema3A OE cells using similar experimental setup as in $(F)$, but with OT-I treatment at day 7 post-injection $(n=6)$. Experiment performed once. ${ }^{* *}=\mathrm{P}<0.001$ by two-way ANOVA.

H. Enumeration of OT-I T cells in tumors (left graph) and their ratio of cells, normalized to the number in the B16.F10 Sema3A KO tumor (right) from same experiment as in (G). ${ }^{*}=$ $P<0.05$ by two-way ANOVA.

Figure 6: CD8+ TILs express NRP1 and are captured in Sema3A rich areas in ccRCC tumors.

A. Correlation of SEMA3A mRNA level with survival of ccRCC patients. Data from TCGA, using TIMER (71). 
B. Schematic representing ccRCC cohort of patient utilized in (C-M).

C. Representative flow cytometric analysis of CD8 and NRP1 expression in PBMC and TILs in cCRCC patient.

D. Analysis of NRP1 expression on CD8+ T cells in PBMC, tumor and tumor-adjacent tissue in $\operatorname{ccRCC}$ cohort $(\mathrm{n}=7-13)$. Bars indicate mean. ${ }^{\star * * *}=\mathrm{P}<0.0001$, ns $=$ not significant by two-way ANOVA.

1249 E. Representative flow cytometric analysis of PD1 and NRP1 on CD8+ TIL in ccRCC patient. F. Analysis of PD1 expression on NRP1 positive CD8+ T cells in PBMC, tumor and tumoradjacent tissue in ccRCC cohort $(n=7-13)$. Bars indicate mean. ns = not significant by twoway ANOVA.

G. Analysis of CDR3 $\beta$ diversity in NRP1 positive $(+)$ and negative (-) CD8+ TILs $(n=4)$. sequences. SI and SA diversity indices (DI) show that in all four patients, NRP1+ TILs are less diverse.

1257 H. Heatmap of TRBV usage in NRP1 positive (+) and negative (-) CD8+ TILs ( $n=4)$. Color indicates relative usage within all of individual patients, as indicated by scalebar.

I. Representative flow cytometric analysis of TCRaß and CT tetramer positive CD8+ TILs (left) and NRP1+CD8+ TILs (right). Error bars indicate mean \pm SD.

J. Graph of percentage CT tetramer positive NRP1+ (red) and NRP1- (blue) CD8+ TILs in four ccRCC patients.

1263 K. Representative CD8 (brown) and CD31 (red) staining in ccRCC tumor. Dashed area in top image indicates zoom area in bottom image. Scalebar, $500 \mu \mathrm{m}$ and $250 \mu \mathrm{m}$.

L. Representative CD31, Sema3A and CD8 staining in Sema3A poor region (top row) and Sema3A rich region (bottom row). Arrows indicate association between Sema3A and CD8 staining. Scalebar, $50 \mu \mathrm{m}$.

M. Enumeration of CD8+ TILs in Sema3A rich (red dots) and poor (blue dots) in patients $1269(n=12)$.

Abbreviations: CT, cancer testis. DI, diversity indices. SA, Shannon index. SI, Simpson Immune Estimation Resource. TRBV, TCR beta chain variable. 


\section{SUPPLEMENTARY DATA}

\section{Supplementary Table 1}

\begin{tabular}{|l|l|l|}
\hline \multicolumn{3}{|l|}{ Baseline characteristics of patient cohort. } \\
\hline Characteristics & $\begin{array}{l}\text { Number } \\
\text { (range) }\end{array}$ & Percent \\
\hline Age (years) & $64.4(42-86)$ & \\
\hline Mean & & \\
\hline Gender & 13 & $56.5 \%$ \\
\hline Male & 10 & $43.5 \%$ \\
\hline Female & & \\
\hline Tumour grade (ISUP) & 0 & 0 \\
\hline 1 & 2 & $8,7 \%$ \\
\hline 2 & 12 & $52.2 \%$ \\
\hline 3 & 6 & $26.1 \%$ \\
\hline 4 & 3 & $13 \%$ \\
\hline N/A & & \\
\hline Tumour location & 14 & $60.9 \%$ \\
\hline Right & 9 & $39.1 \%$ \\
\hline Left & 17 & \\
\hline Type of surgery & 17 & $26.9 \%$ \\
\hline Radical nephrectomy & 6 & $26.1 \%$ \\
\hline Partial nephrectomy & 5 & \\
\hline Tumour stage & 1 & \\
\hline pT1a & & \\
\hline pT1b & & \\
\hline pT2a & & \\
\hline pT2b & & \\
\hline & & \\
\hline & & \\
\hline
\end{tabular}




\begin{tabular}{|l|l|l|}
\hline pT3a & 14 & $63.6 \%$ \\
\hline pT3b & 0 & 0 \\
\hline pT3c & 1 & $4.5 \%$ \\
\hline pT4 & 0 & 0 \\
\hline N/A & 1 & $4.5 \%$ \\
\hline
\end{tabular}

1279

1280 


\section{Supplementary Figure 1}

A

Data from Best et al.

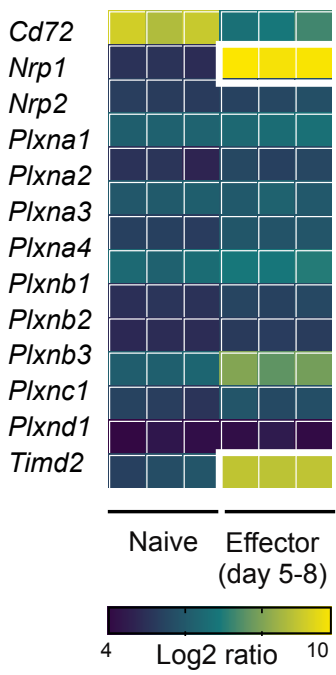

D
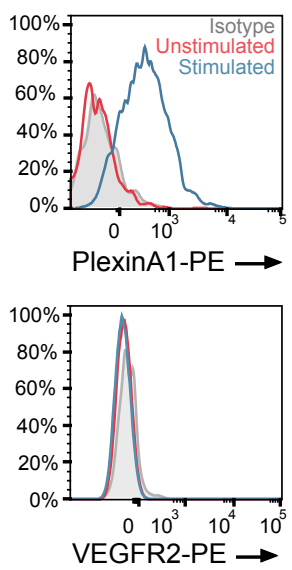

B Oh $24 \mathrm{~h} 48 \mathrm{~h} 72 \mathrm{~h} \quad 96 \mathrm{~h}$
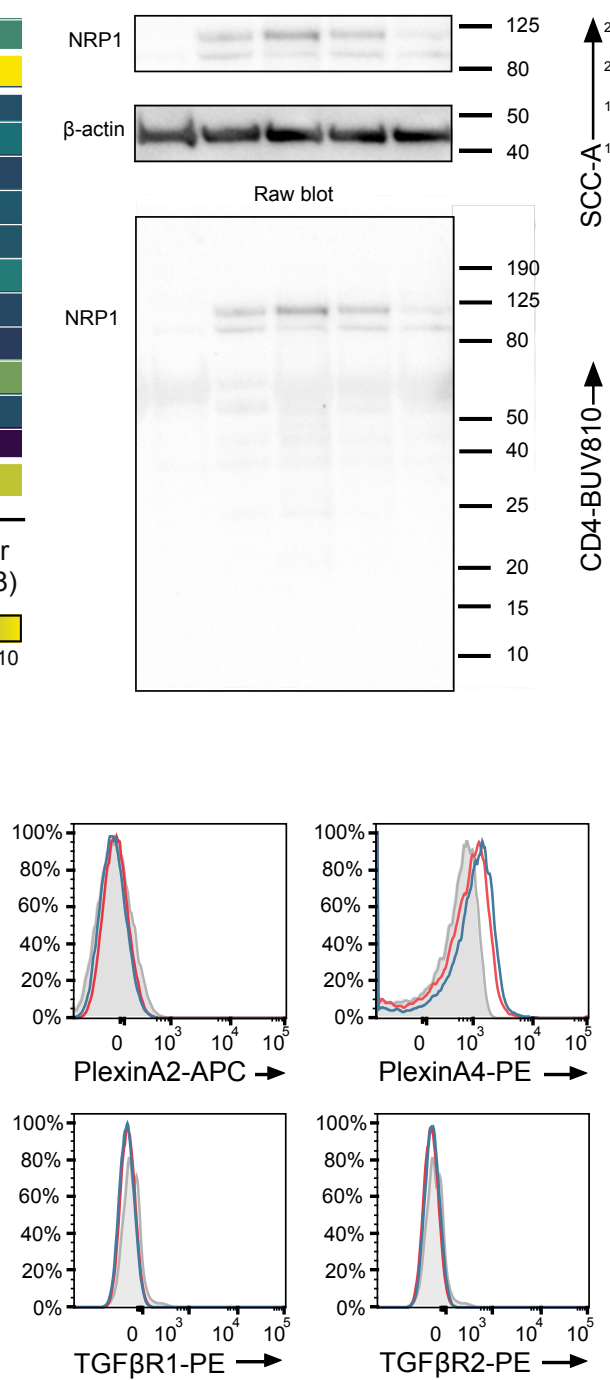

C
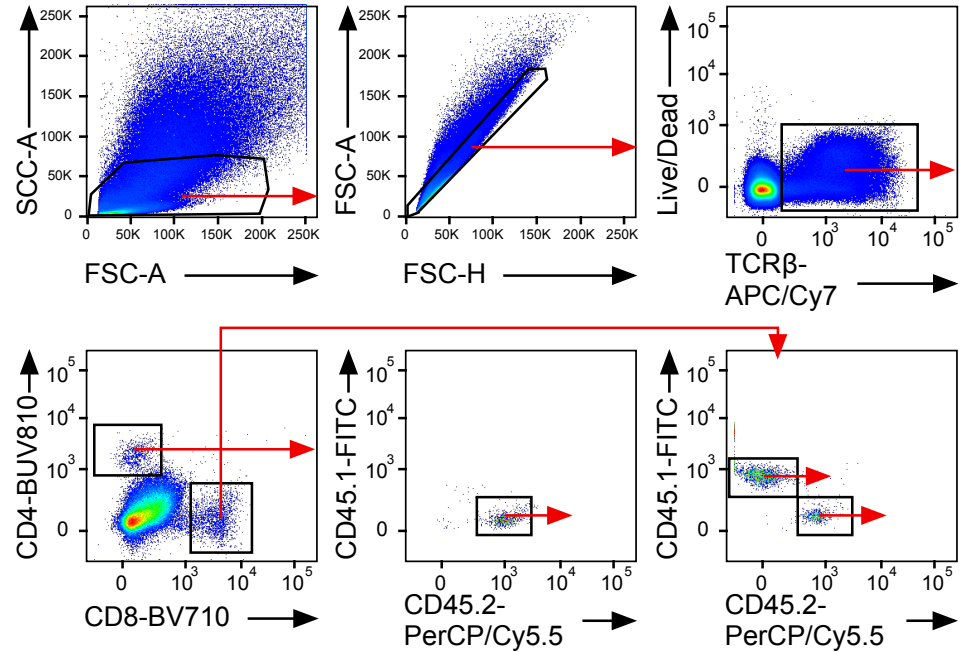

E

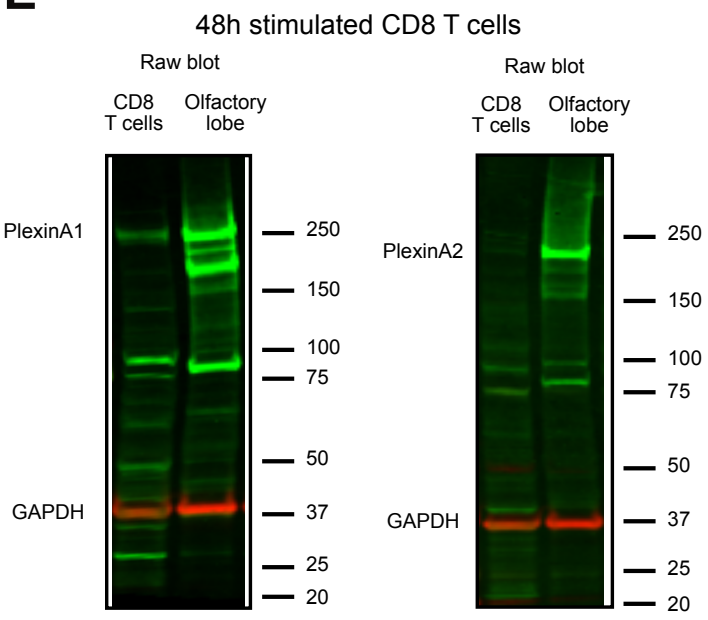

$\mathbf{F}$

B16.F10.Ova

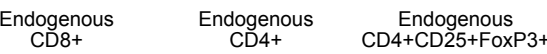

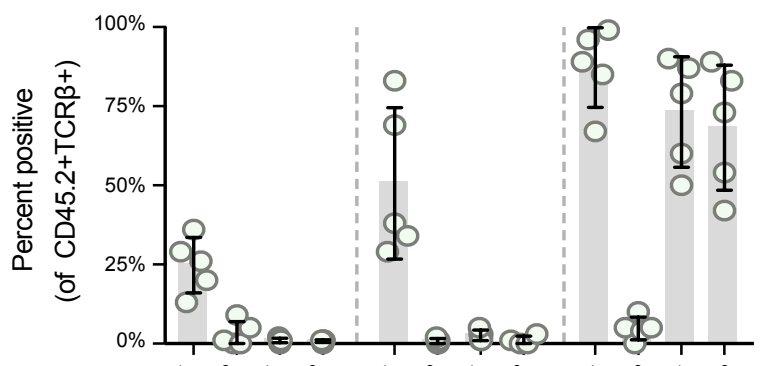

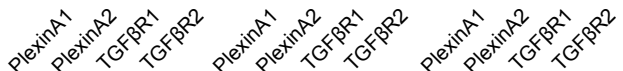

NRP1 Co-receptors 


\section{Supplementary Figure 1 (relates to Figure 1).}

1282 A. Heatmap of transcript levels of known semaphorin receptors on naïve and effector OT-I 1283 T cells following infection with vaccinia-OVA. Data from Best et al. 2013 (16).

1284 B. Western blot showing NRP1 up-regulation in OT-I T cells following stimulation with 1285 SIINFEKL. Experiment was performed once.

1286 C. Gating strategy for Figure 1F-G.

1287 D. Flow cytometric analysis of Plexin-A1, Plexin-A2, Plexin-A4, VEGFR2, TGF $\beta$ R1 and 1288 TGF $\beta R 2$ on unstimulated and 48 hour SIINFEKL stimulated OT-I T cells. Experiment 1289 representative of three.

1290 E. Western blots showing expression of Plexin-A1 (left, green) and Plexin-A2 (right, green) 1291 in 48 hour stimulated OT-I T cells and olfactory lobe (positive control). Experiment performed 1292 once. Loading control GAPDH shown in red.

1293 F. Flow cytometric analysis of Plexin-A1, Plexin-A2, TGF $\beta R 1$ and TGF $\beta R 2$ expression on 1294 OT-I T cells and endogenous CD8+ TILs, 11 days after adoptive transfer og OT-I T cells in 1295 antigen-expressing tumor (B16.F10.Ova) (n=5). Error bars indicate SD. 


\section{Supplementary Figure 2}

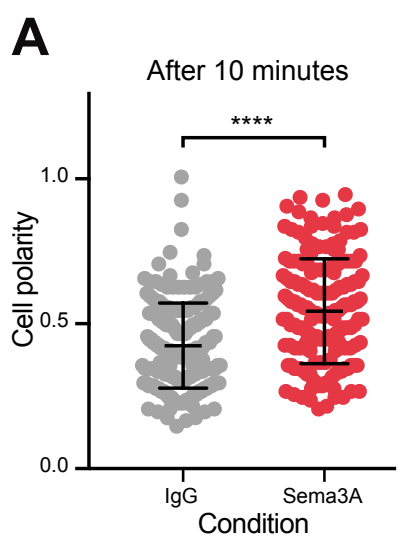

B
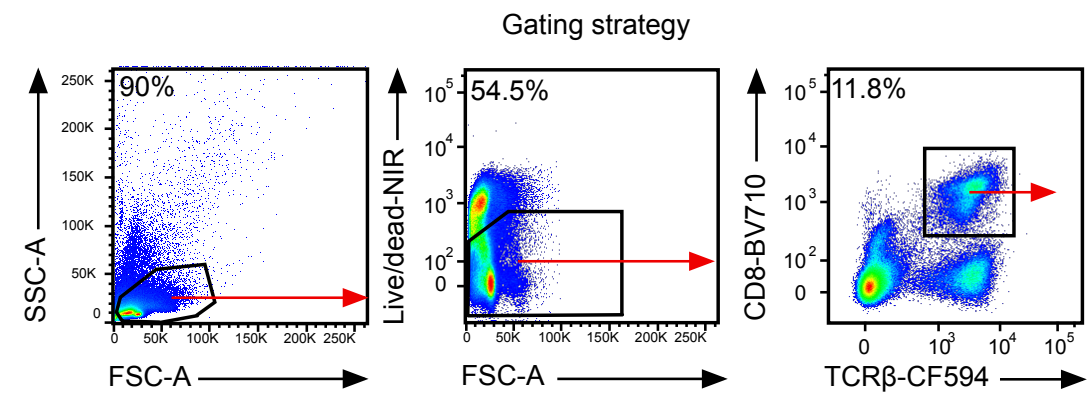

Expression of selectins and integrins following Sema3A treatment
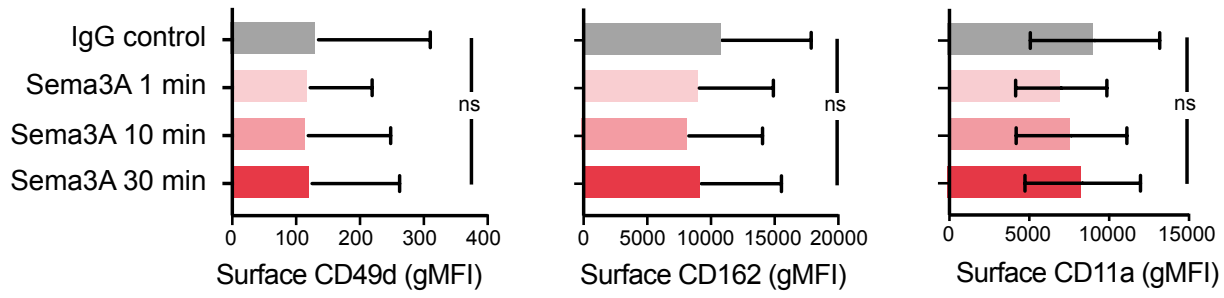


\section{Supplementary Figure 2 (relates to Figure 2).}

1299 A. Relative frequency of cell polarity of 48 hour stimulated OT-I T cells treated with IgG or 1300 Sema3As-p. A polarity of 1 indicates a shape of a perfect circle, 0 a rectangular shape. 1301 Experiment repeated three times. ${ }^{* * *}=\mathrm{P}<0.0001$ by Student's t-test.

1302 B. Gating strategy for analyzing 48 hour stimulated OT-I splenocytes treated with Sema3As$1303 \mathrm{P}$ (top). Bar graphs of gMFI of CD49d, CD162 and CD11a following Sema3As-p treatment at 1304 indicated times. ns = not significant, by Kruskal-Wallis test. 


\section{Supplementary Figure 3}

A
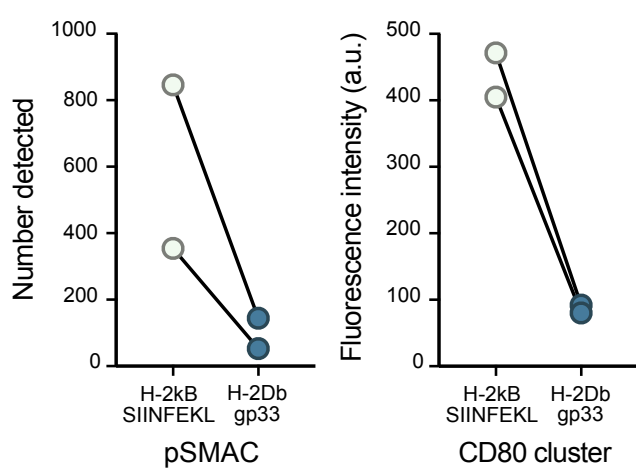

B

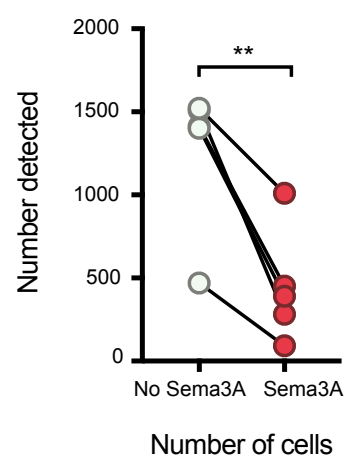

C

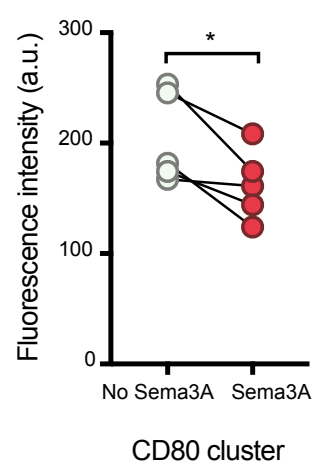

D

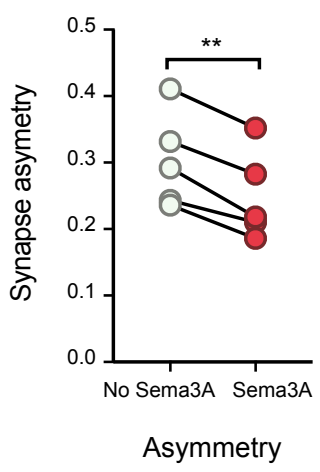

E

Cell-cell conjugation after 15 minutes
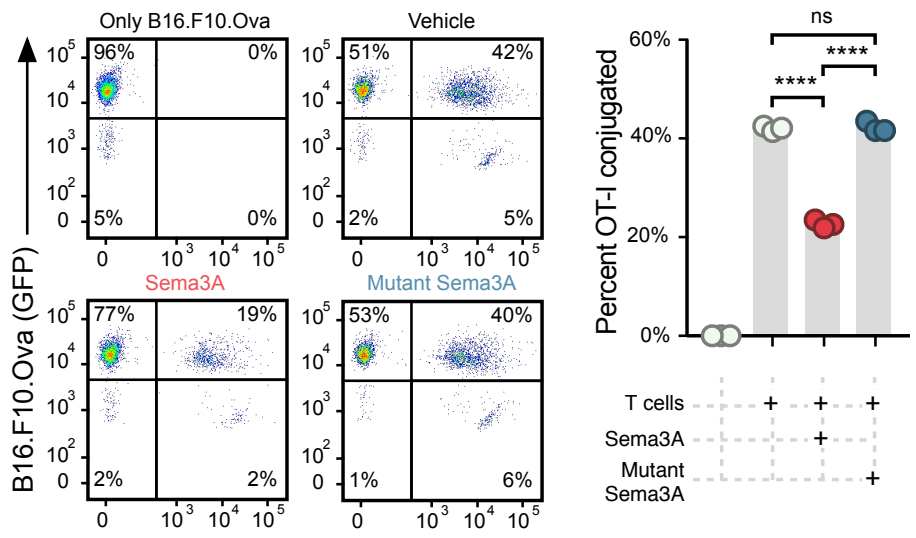

$\mathbf{F}$

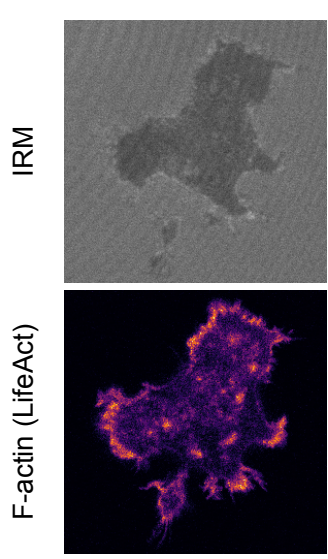

IRM and F-actin overlay

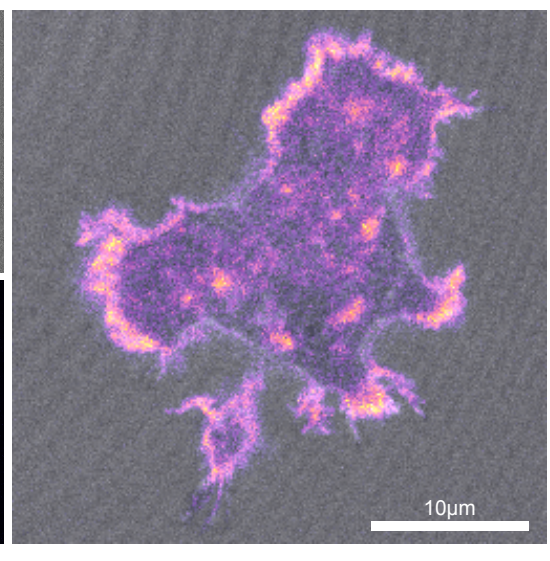




\section{7}

1308

1309

1310

1311

1312

1313

1314

1315

1316

1317

1318

1319

1320

1321

1322

1323

1324

1325

1326

1327

1328

1329

1330

1331

\section{Supplementary Figure 3 (relates to Figure 3-4).}

A. Quantification of pSMAC (left) and CD80 clustering (right) in immunological synapses of 48 hour stimulated OT-I T cells when presented with a relevant (H-2Kb-SIINFEKL) or irrelevant (H-2D-gp33) MHC monomer loaded onto the bilayer.

B. Quantification of 48 hour stimulated OT-I T cells detected in high-throughput assay with or without Sema3As-P-ı pre-treatment. ${ }^{*}=\mathrm{P}<0.01$, by paired t-test.

C. Fluorescence intensity of CD80 signal introduced by 48 hour stimulated OT-I T cells in high-throughput assay with or without Sema3As-P-I pre-treatment. ${ }^{*}=\mathrm{P}<0.05$, by paired ttest.

D. Analysis of radial symmetry of synapses in OT-I T cells in high-throughput assay with or without Sema3As-P-I pre-treatment. Asymmetry of the synapse is quantified as the distance between the centroids of the CD80 cluster and that of the PSMAC relative to the diameter of the cell. ${ }^{\star}{ }^{\star}=\mathrm{P}<0.01$, by paired t-test.

E. Gating strategy and representative images showing number of B16.F10.Ova cells and T cells as either singlets or doublets under four different conditions: cancer cells alone, with normal media, media with Sema3As-p or with mutant Sema3A (left). Quantification of 3 biological replicates, showing approximately $50 \%$ reduction in cell-cell conjugation when Sema3A is present (right). Data representative of three independent experiments. ${ }^{* \star *}=\mathrm{P}$ $<0.0001$, ns $=$ not significant, by two-way ANOVA. Gray bars indicate mean.

F. Images from live-cell imaging of OT-I $\times$ LifeAct $T$ cells showing concordance between IRM shadow and F-actin signal. Scalebar $10 \mu \mathrm{m}$.

Abbreviations: a.u., arbitrary unit. 
A

CD4-Cre $x_{\text {NRP1 }}{ }^{+/+} \quad$ CD4-Cre $x$ NRP1 ${ }^{\text {Flox/+ }} \quad$ CD4-Cre $x$ NRP1 Flox/Flox

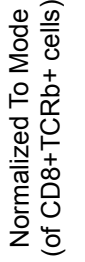
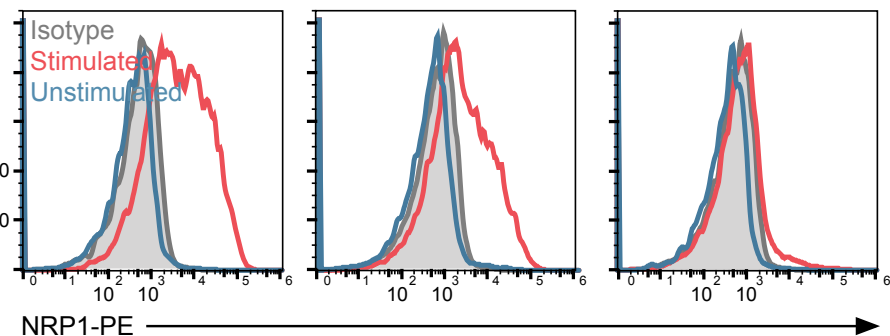

B

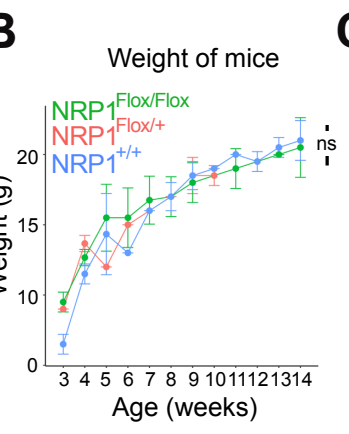

C

Spleen NRP1-PE

D

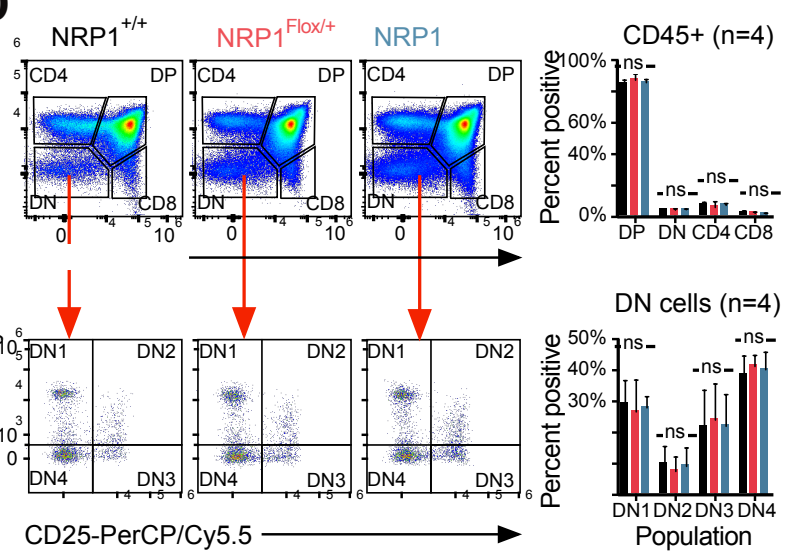

$\mathbf{F}$

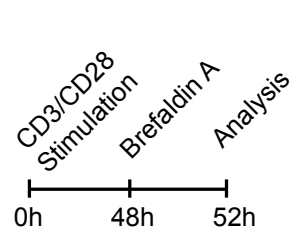

G
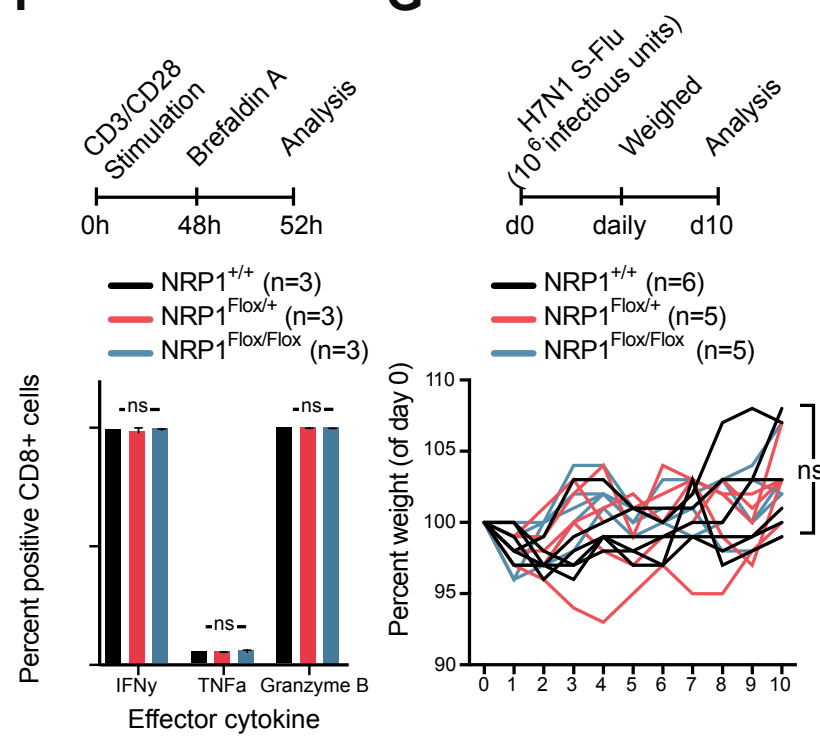

K

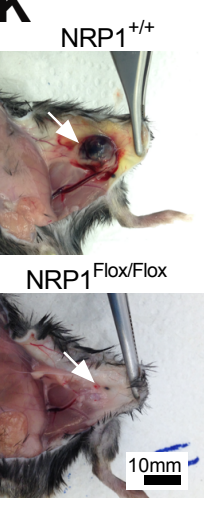

L

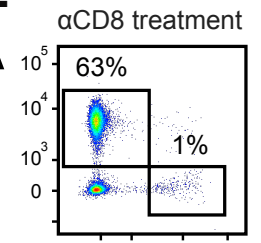

Isotype control
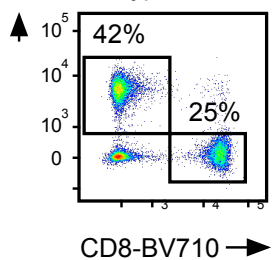

- Day 15 post -

M
E
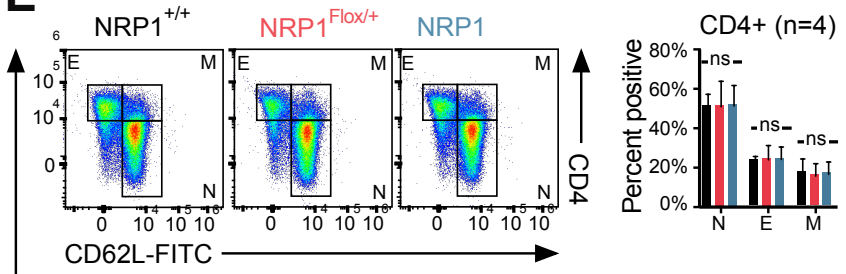

$\mathrm{CD} 8+(\mathrm{n}=4)$
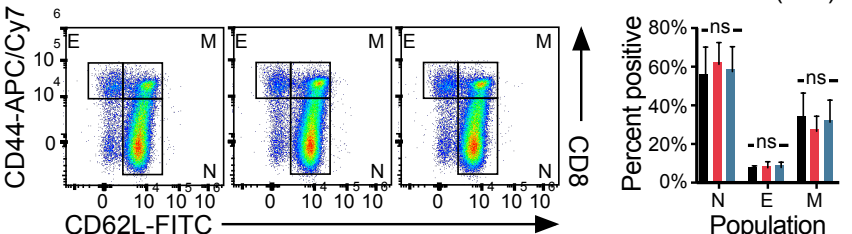

H
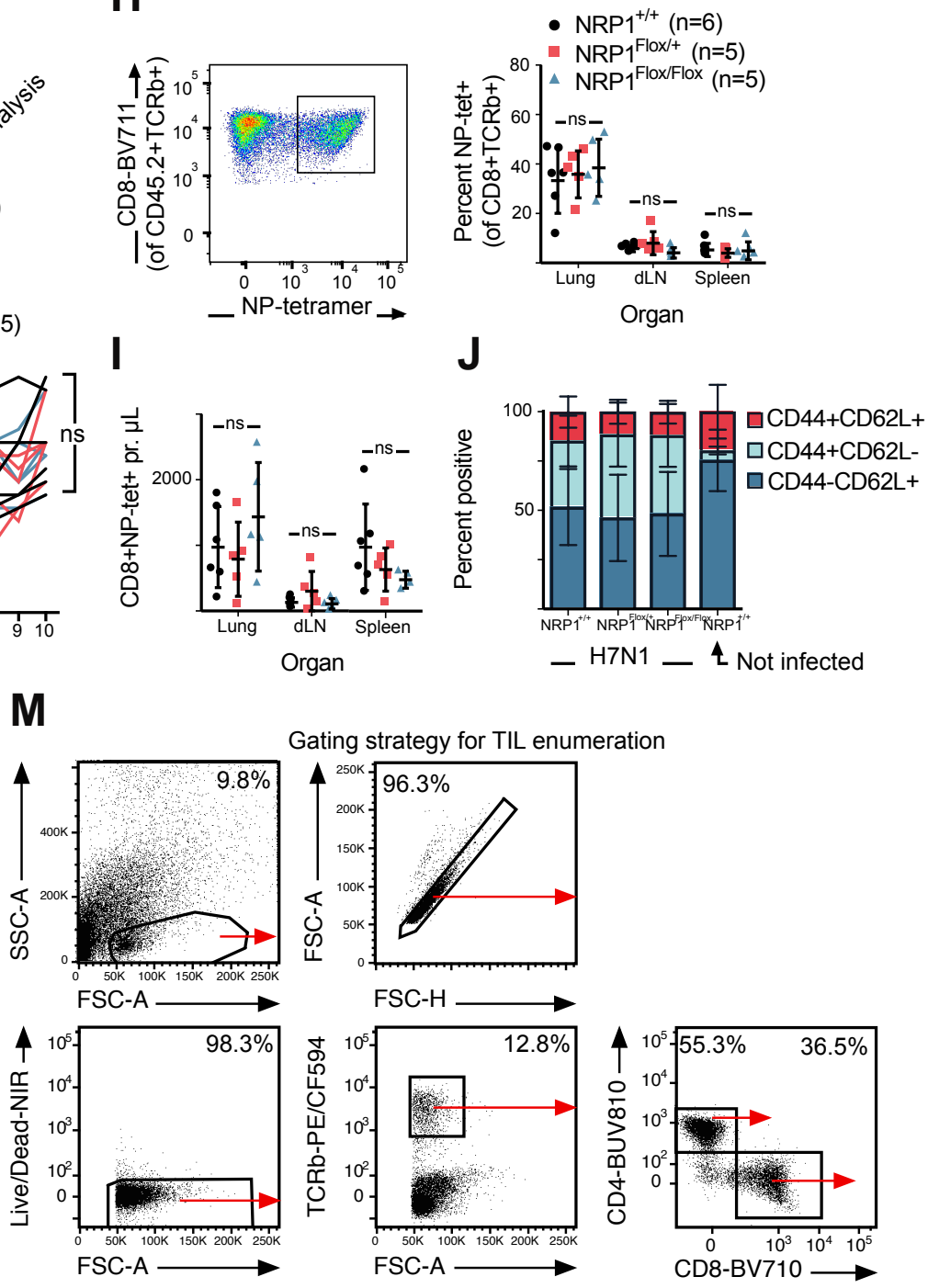

Gating strategy for TIL enumeration
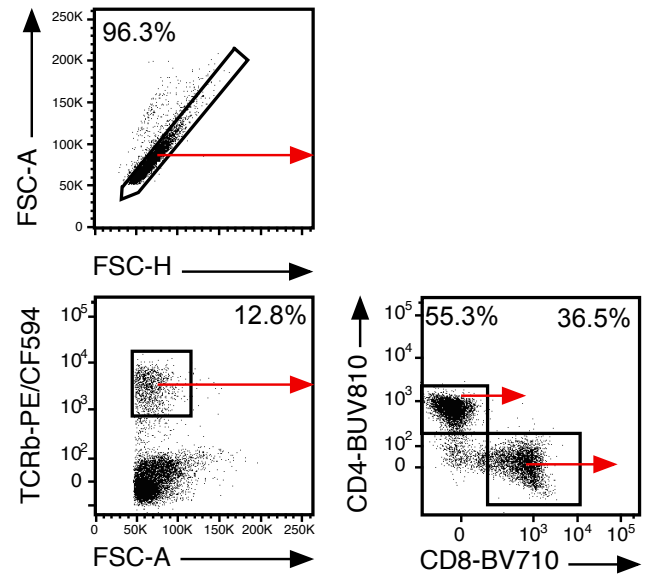


\section{2}

1333

1334

1335

1336

1337

1338

1339

1340

1341

1342

1343

1344

1345

1346

1347

1348

1349

1350

1351

1352

1353

1354

1355

1356

1357

1358

1359

1360

1361

\section{Supplementary Figure 4 (relates to Figure 5).}

A. Flow cytometric analysis of naïve or $C D 3 / C D 28$ stimulated splenocytes from either Nrp1 ${ }^{1++}, \mathrm{Nrp} 1^{\mathrm{Flox} /+}$ or Nrp1 $1^{\text {Flox/Flox }}$ mice. Cells are gated on CD8 and TCR $\beta$. Experiment performed three times.

B. The weight of female littermates $(n=17)$ were followed for 12 weeks and revealed no difference between genotypes. Data indicate mean \pm SD. ns $=$ not significant by two-way ANOVA.

C. The size of spleens from female littermate mice of different genotypes at 12 weeks of age.

D. Representative plots showing the distribution of double negative, double positive, CD4 and CD8 positive thymocytes (upper panel, left) and DN1, DN2, DN3 and DN4 populations

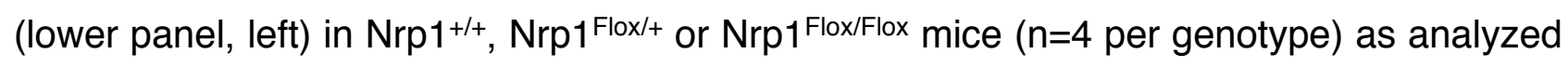
by flow cytometry. Cells are gated on CD45.2. Quantification of cell populations in different genotypes (upper and lower histograms, right). Experiment performed once. ns $=$ not significant by two-way ANOVA.

E. Representative plots showing T cell effector populations in splenic CD4+ (top) and CD8+ (bottom) T cells. Cells are gated on CD45.2 and TCR $\beta$ ( $n=4$ per genotype). Data combined from two independent experiments. ns = not significant by two-way ANOVA.

F. Cytokine production following ex vivo stimulation by CD3/CD28. Experimental design (upper panel). Quantification of IFNY, TNFa and Granzyme B by intracellular staining (lower panel). Cells are gated on TCR $\beta$ and $C D 8$ ( $n=3$ mice per genotype). Experiment repeated twice. ns = not significant by two-way ANOVA.

G. Weight of mice following H7N1 S-Flu infection. Experimental design (upper panel). Weight of mice, normalized to day 0 of individual mouse weight (lower panel). Experiment performed once ( $n=5-6$ mice per genotype). $n s=$ not significant by two-way ANOVA.

H. Analysis of H7N1 S-Flu-specific T cells 10 days post-infection. Example H-2DB-NP tetramer staining in lung of infected mouse (left figure). Quantification of H-2DB-NP tetramer positive CD8+ T cells in lung, dLN and spleen (right figure). Cells are gated on CD45.2, TCR $\beta$ and CD8 ( $n=5-6$ per genotype). Experiment performed once. $n s=$ not significant by two-way ANOVA. 
1362 I. Quantification of total number of infiltrating H-2DB-NP tetramer positive CD8+ in lung, dLN 1363 and spleen 10 days post-infection. Experiment performed once. ns = not significant by two1364 way ANOVA.

$1365 \mathrm{~J}$. Analysis of effector subpopulations in lung 10 days post-infection in different genotypes 1366 of mice ( $n=5-6$ mice per genotype).

1367 K. Representative image of B16.F10 tumors 15 days post-injection in Nrp1+/+ (upper image) 1368 and Nrp1Flox/Flox (lower image) mice. Arrows indicates tumors. Scalebar $10 \mathrm{~mm}$.

1369 L. Representative flow cytometric analysis of peripheral blood in mice treated with either 1370 aCD8 antibodies (upper scatterplot) or isotype control (lower scatterplot).

1371 M. Gating strategy used for flow cytometric analysis of TIL enumeration in mice.

1373 Abbreviations: dLN, draining lymph node. DN, double negative. E, effector T cells. N, naïve 1374 T cells. M, memory T cells. TIL, tumor-infiltrating leukocytes. 


\section{Supplementary Figure 5}

A

Gating strategy
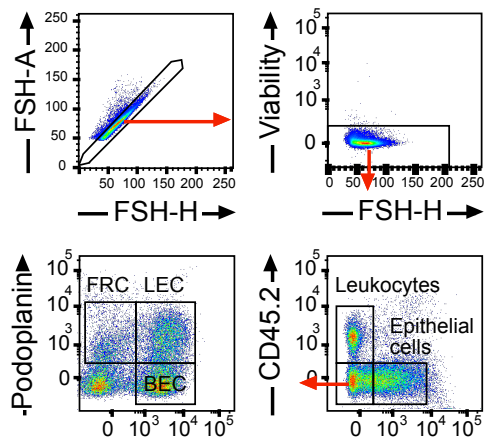

$-\mathrm{CD} 31 \rightarrow$
B

Sema3A expression in lung before and after H7N1 S-Flu infection

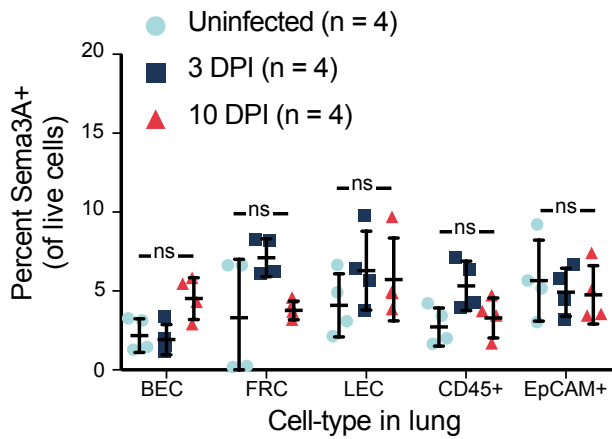

Sema3A expression in draining lymph node before and after H7N1 S-Flu infection

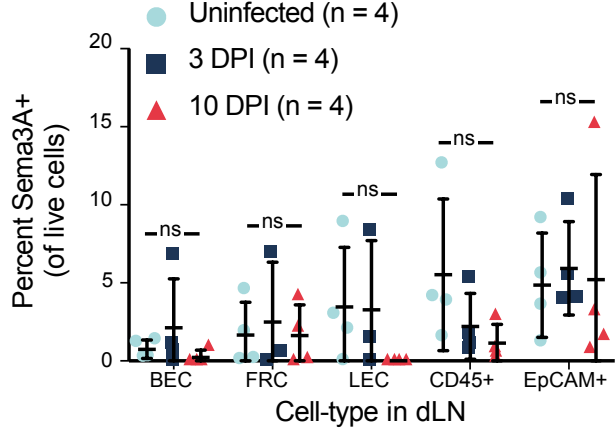

C

Sema3A transcript level under hypoxic condition $\left(1 \% \mathrm{O}_{2}\right)$

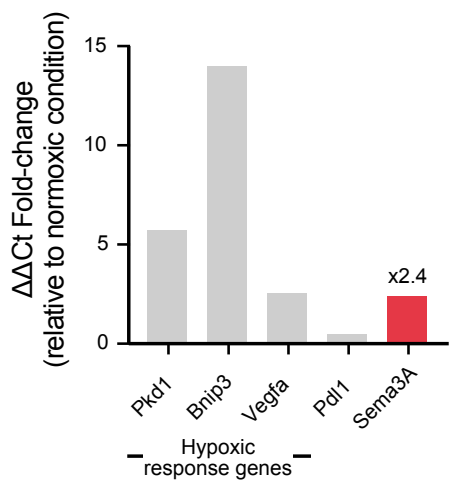

$\mathbf{E}$

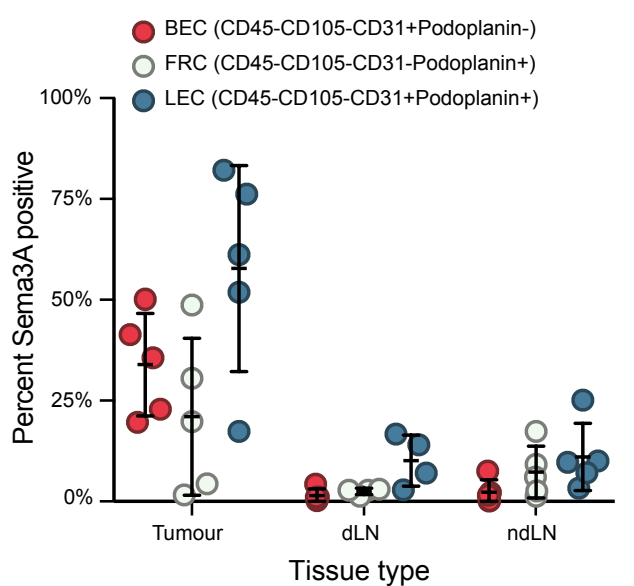

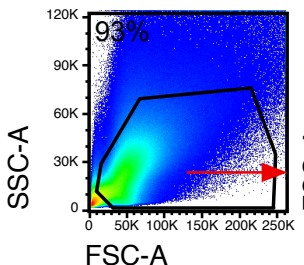

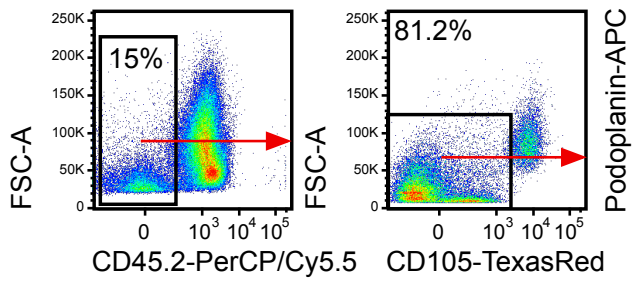

$\mathbf{F}$
Gating strategy

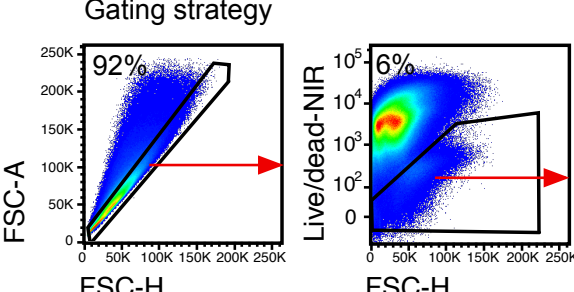

FSC-H

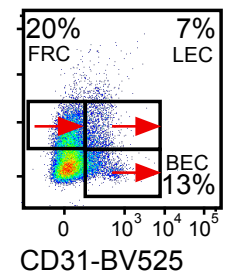

CD31-BV525

Sema3A expression in B16.F10 TME

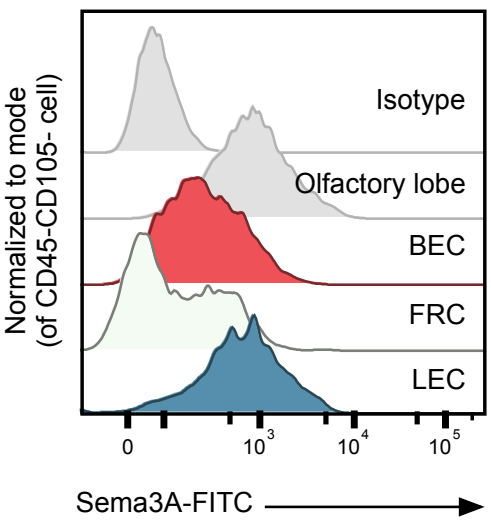

G

Murine Sema3A

Sema3A transcript level

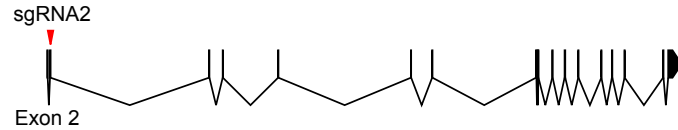

Deep-sequencing B16.F10.Ova Sema3A KO Clone

bold substitutions
$\square$ Insertions
Dething

--. Preedicted cleavage position

GAAAGAACAATGTGCCAAGACTGAAATTATCGTACAAAGG Reference

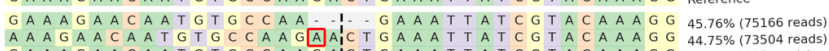

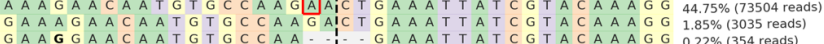

GAAGGAACAATGTGCCAA. GAATTATCGTACAAAGG $0.22 \%$ (1354 reads)

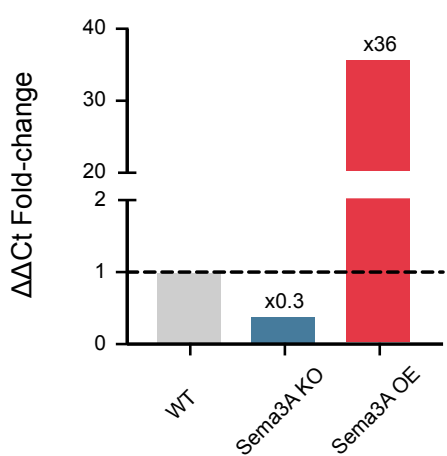

$H$

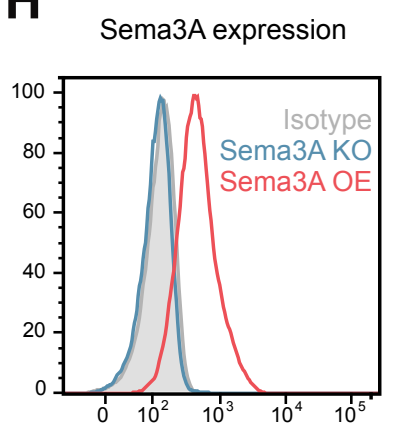

Sema3A-PE

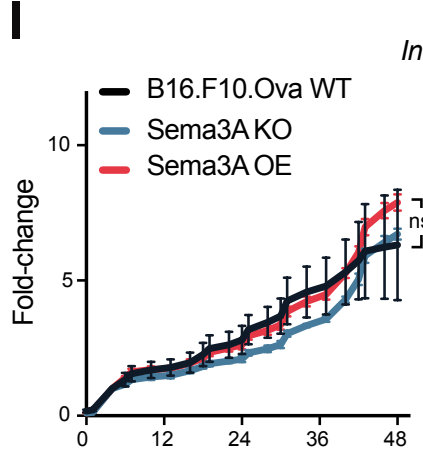

In vitro growth of B16.F10.Ova cell-lines 


\section{Supplementary Figure 5 (relates to Figure 5).}

1378 A. Gating strategy for analyzing Sema3A expression among leukocytes, epithelial and 1379 endothelial cells in lung and dLN.

1380 B. Quantification of Sema3A positive cells in different cell populations in uninfected $(n=4)$, 1381 and infected mice, at 3 days DPI $(n=4)$ and 10 DPI $(n=4)$ in lung (left panel) or dLN (right 1382 panel). Nrp1 $1^{\text {Flox/Flox }}$ mice used. Data combined from two independent experiments. ns = not 1383 significant by two-way ANOVA.

1384 C. Quantification of Pkd1, Bnip3, Vegfa, Pdl1 and Sema3A mRNA level following 24 hour 1385 culture in 1\% O2 chamber.

1386 D. Gating strategy (left) and representative histograms (right) analyzing Sema3A positive 1387 cell populations in B16.F10 tumors 11 days post-injection. Olfactory lobe is used as a 1388 positive control for Sema3A expression.

1389 E. Quantification of Sema3A positive cells in same experiment as in (D) in tumor, dLN and 1390 ndLN.

1391 F. Genomic organization of murine Sema3a gene, indicating where CRISPR guide RNA 1392 targets with red arrow (upper figure). MiSEQ sequence results for chosen Sema3A KO clone 1393 showing 4 base deletion in two alleles (46\% of all reads), a frameshift in one allele (45\% of 1394 all reads) and WT reads in $2 \%$ of all reads.

1395 G. RT-qPCR analysis show down- and up-regulation of Sema3A in Sema3A KO and OE 1396 cell lines, respectively. Normalized to Hprt. Experiment performed once.

1397 H. Intracellular staining shows no detectable expression of Sema3A in Sema3A KO cells, 1398 and expression in Sema3A OE cells, as expected. Experiment performed once, at low 1399 seeding density.

1400 I. Growth of WT, Sema3A KO and Sema3A OE B16.F10.Ova cell lines in normal, IFNY or 1401 TNFa-rich media. Experiment performed once. Data indicate mean \pm SD. ns = not significant 1402 by two-way ANOVA.

1404 Abbreviations: BEC, blood endothelial cells. dLN, draining lymph node. DPI, days post1405 infection. FRC, fibroblastic reticular cells. KO, knockout. LEC, lymphatic endothelial cells. 1406 ndLN, non-draining lymph node. OE, overexpressing. TME, tumor microenvironment. WT, 1407 wild-type. 


\section{Supplementary Figure 6}

A

Gating strategy for analysing TILs
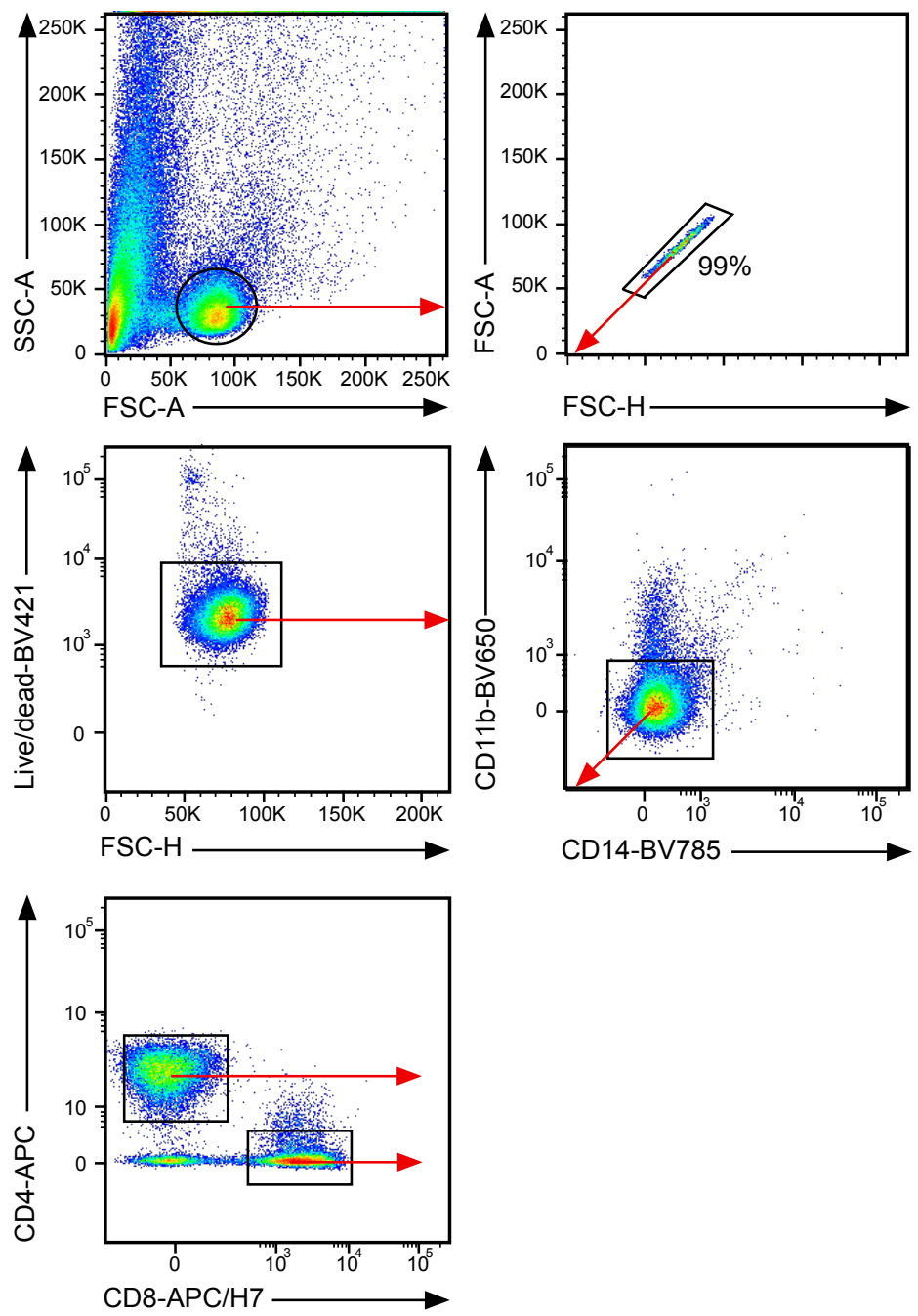

B Gating strategy for single cell sorting and CT-tetramer analysis

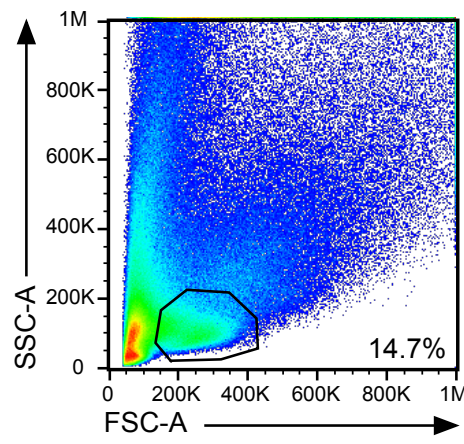

1

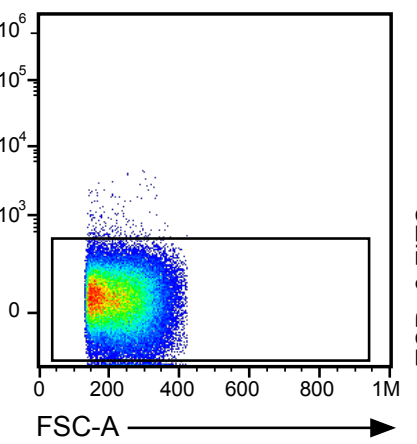

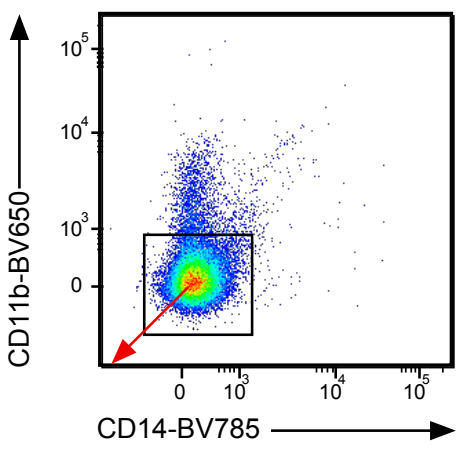
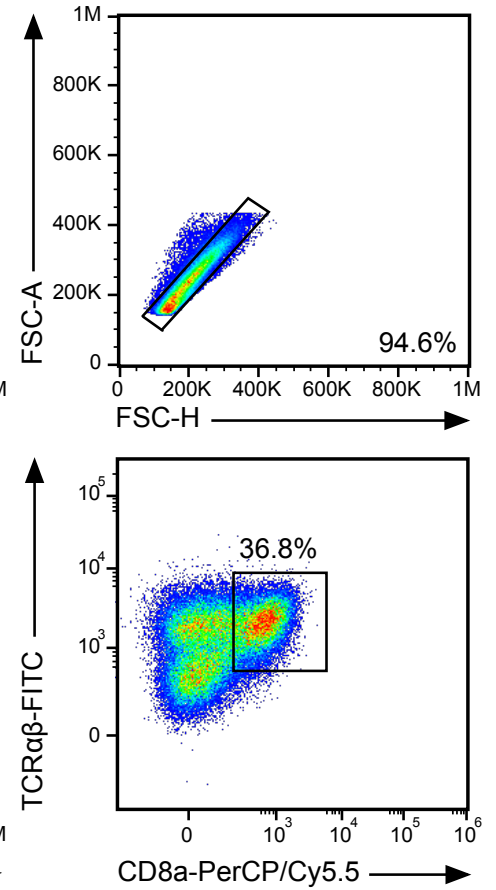

C

Sema3A expression in kidney tissue

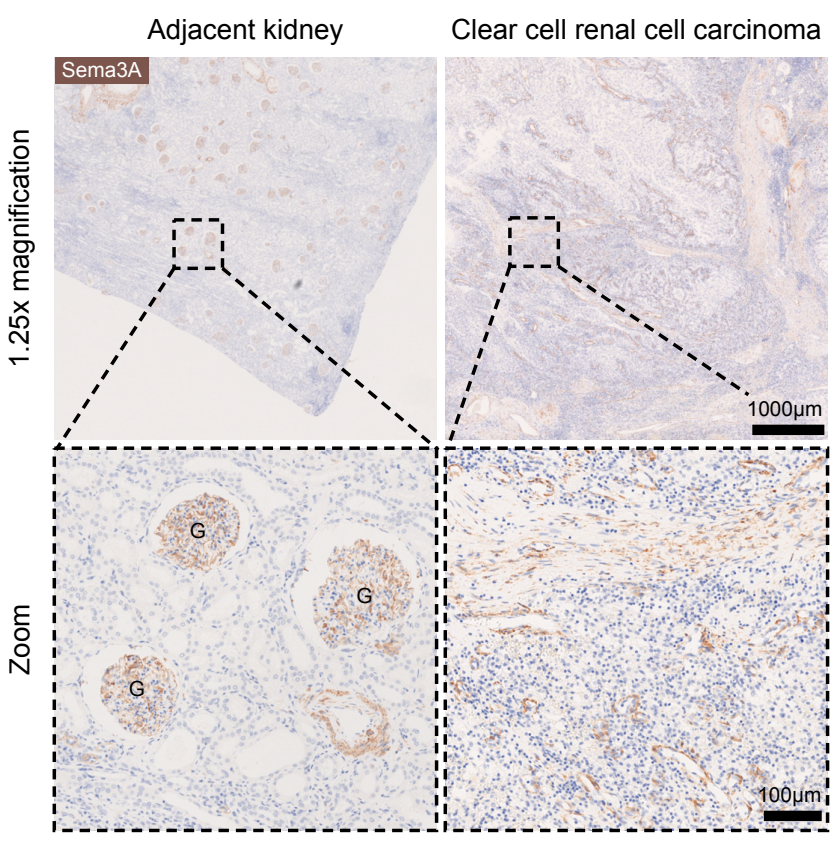

D

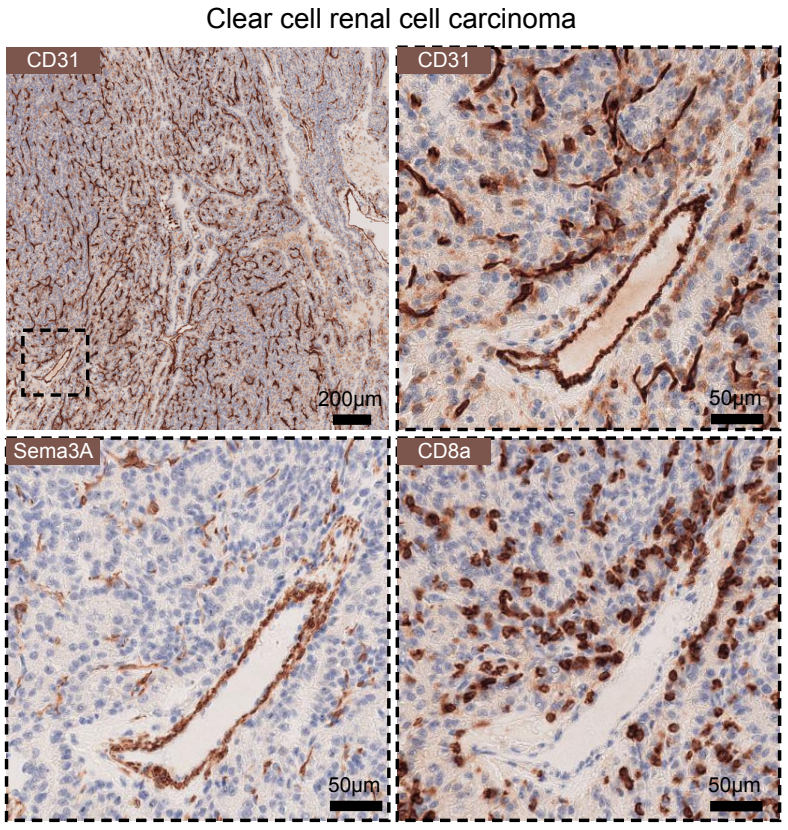

E

$\mathbf{F}$
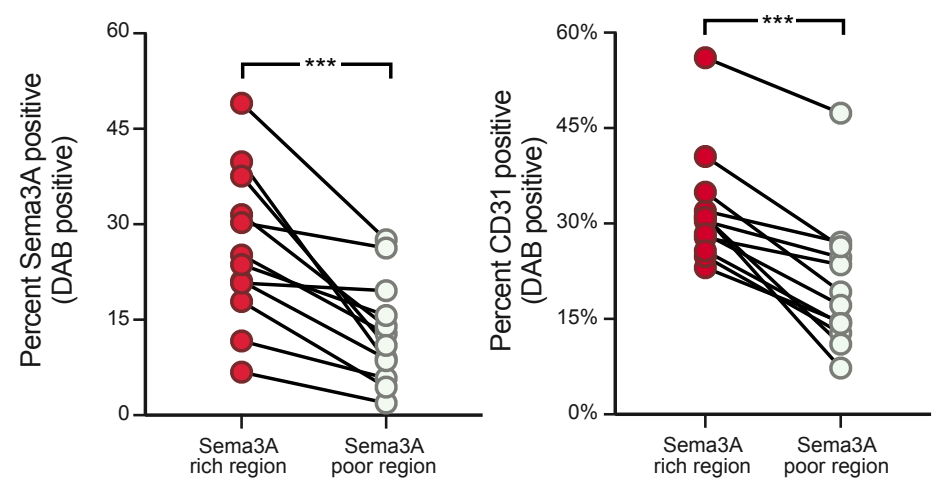


\section{Supplementary Figure 6 (relates to Figure 6).}

1409 A. Gating strategy for analyzing TILs from ccRCC patients.

1410 B. Gating strategy for single cell sorting and CT-tetramer analysis.

1411 C. Sema3A expression in tumor-adjacent and tumor tissue of ccRCC patient. G indicates 1412 kidney glomeruli. Scalebars indicate $1000 \mu \mathrm{m}$ (upper row) and $100 \mu \mathrm{m}$ (lower row).

1413 D. Serial sections from ccRCC tumor stained for CD31, Sema3A and CD8a. Dashed box in 1414 upper left image indicates the region depicted at higher magnification in the three other 1415 images. Scalebar indicates $200 \mu \mathrm{m}$ (upper left) and $50 \mu \mathrm{m}$ (other images).

1416 E. Expression of CD31 in Sema3A rich and poor regions. ${ }^{* * *}=\mathrm{P}<0.0001$ by paired t-test.

1417 F. Expression of Sema3A in selected Sema3A rich and poor regions. ${ }^{* * *}=\mathrm{P}<0.0001$ by 1418 paired t-test.

1420 Abbreviations: ccRCC, clear cell renal cell carcinoma. DAB, 3,3'-Diaminobenzidine. 